\title{
1,2-Dioxines Containing Tethered Hydroxyl Functionality as Convenient Precursors for Pyran Syntheses.
}

Thomas D. Avery ${ }^{\dagger}$,Daniela Caiazza ${ }^{\dagger}$, Julie A. Culbert ${ }^{\dagger}$, Dennis K. Taylor** ${ }^{\dagger}$, Edward R. T. Tiekink $k^{\ddagger}$

${ }^{\dagger}$ Department of Chemistry, The University of Adelaide, South Australia 5005, Australia. ${ }^{\ddagger}$ School of Science, Griffith University, Nathan, Queensland 4111, Australia.

\section{Experimental Section}

$\begin{array}{ll}\text { General Methods } & \text { S3 }\end{array}$

General procedure for Wittig reaction: Synthesis of 5a-d. $\quad$ S3

General procedure for formation of $\alpha, \beta$-unsaturated esters 30a-c. $\quad$ S6

$\begin{array}{ll}\text { General procedure for formation of } \alpha, \beta \text {-unsaturated alcohols 31a-c. } & \text { S7 }\end{array}$

General procedure for formation of $\alpha, \beta$-unsaturated aldehydes 6a-c. $\quad$ S8

4-\{[1-(tert-Butyl)-1,1-dimethylsilyl $]$ oxy $\}$ butyl)(triphenyl)phosphonium iodide 7 S9

$\begin{array}{ll}\text { General procedure for formation of protected dienes 8a-c. } & \text { S9 }\end{array}$

$\begin{array}{ll}\text { General procedure for TBDMS cleavage: Synthesis of 9a-c. } & \text { S10 }\end{array}$

$\begin{array}{ll}\text { General procedure for the synthesis of 1,2-dioxines 10a-g. } & \text { S13 }\end{array}$

General procedure for the synthesis of pyrans 12a, 13a. S16

$\begin{array}{ll}\text { Crystallographic data for 16d. } & \text { S18 }\end{array}$

General procedure for the synthesis of 1,4-diketones 14a, 17a-d, $23 . \quad$ S20

3-(5-Phenyl-2-furyl)-1-propanol 18a. S23

General procedure for the synthesis of trans $\gamma$-hydroxy enones 11a-g. $\quad$ S23

$\begin{array}{ll}\text { References } & \text { S25 }\end{array}$ 


\section{NMR Spectra}

${ }^{13} \mathrm{C}$ NMR

$10 a$

S26

$10 b$

S27

10c

S28

10d

S29

10e

S30

$10 f$

S31

$10 \mathrm{~g}$

S32

$12 \mathrm{a}$

S33

13a

S34

$15 a$

S35

$15 b$

S36

$15 \mathrm{c}$

S37

15d

S38

$15 \mathrm{e}$

S39

$16 a$

S40

$16 \mathrm{~b}$

S41

$16 \mathrm{c}$

S43

16d

S44

$16 \mathrm{e}$

S45

$14 a$

$17 \mathbf{a}$

$17 \mathrm{c}$

17d

$18 a$

19

S46

S47

S48

S49

S50

20

S51

21

S52

S53

22

S54

23

S55

24

S56

25

S57

26

S58

27

S59

${ }^{1} \mathrm{H}$ NMR

17b

S60 
General Methods. Solvents were dried by appropriate methods wherever needed. Thin-layer chromatography (TLC) used aluminium sheets coated with silica gel 60 $\mathrm{F}_{254}(40 \times 80 \mathrm{~mm})$ and visualized under $254 \mathrm{~nm}$ light, or developed in vanillin or permanganate dip. Flash chromatography was conducted using silica gel 60 of particle size 0.040-0.063 mm. Melting points were uncorrected. ${ }^{1} \mathrm{H}$ NMR and ${ }^{13} \mathrm{C}$ NMR spectra were recorded in $\mathrm{CDCl}_{3}$ solution at either $300 \mathrm{MHz}$ or $600 \mathrm{MHz}$ instrument, TMS (0 ppm) and $\mathrm{CDCl}_{3}(77.0 \mathrm{ppm})$ were used as internal standards. All yields reported refer to isolated material judged to be homogeneous by TLC and NMR spectroscopy.

General procedure for Wittig reaction: Synthesis of 5a-d. Potassium tertbutoxide (1.05 equiv) was added to a suspension of triphenyl[(E)-3-phenyl-2propenyl]phosphonium chloride 3 (1.1 equiv) in anhydrous diethyl ether ( $10 \mathrm{~mL} / \mathrm{g}$ of 3) at $22^{\circ} \mathrm{C}$ and under an inert atmosphere $\left(\mathrm{N}_{2}\right)$. After stirring for 15 minutes the solution was cooled to $0^{\circ} \mathrm{C}$ and a solution of aldehyde 4 (1 equiv) in anhydrous diethyl ether or THF (10 mL / g of 4) was added. After stirring for 48 hours at $22^{\circ} \mathrm{C}$ the solution was diluted by half with hexanes and filtered through silica $(3 \mathrm{~cm})$. The silica was washed with 2:3 ethyl acetate/hexanes until all diene had been removed. The volatiles were then removed from the combined filtrates in vacuo were taken to dryness and the resulting residue was purified by flash chromatography to give desired dienes 5a-d.

7-Phenyl-4,6-heptadien-1-ol (5a) Yield: 9.96 g, 64\%; Colourless oil consisting of a 2:3 mixture of $4 Z, 6 E$ and $4 E, 6 E$ isomers. $(4 Z, 6 E)-7-p h e n y l-4,6-h e p t a d i e n-1-o l ~ R_{f}$ 0.43 (2:3 ethyl acetate/hexanes); ${ }^{1} \mathrm{H} \mathrm{NMR}(600 \mathrm{MHz}) \delta 1.71$ (quin, $\left.J=6.6 \mathrm{~Hz}, 2 \mathrm{H}\right)$, 
$2.11(\mathrm{br} \mathrm{s}, 1 \mathrm{H}), 2.39(\mathrm{dq}, J=6.6,1.2 \mathrm{~Hz}, 2 \mathrm{H}), 3.69(\mathrm{t}, J=6.6 \mathrm{~Hz}, 2 \mathrm{H}), 5.53(\mathrm{dt}, J=$ 10.2, $6.6 \mathrm{~Hz}, 1 \mathrm{H}), 6.19$ (ddd, $J=11.4,10.2,1.2, \mathrm{~Hz}, 1 \mathrm{H}), 6.54(\mathrm{~d}, J=15.6 \mathrm{~Hz}, 1 \mathrm{H})$, 7.08 (ddd, $J=15.6,11.4,1.2 \mathrm{~Hz}, 1 \mathrm{H}), 7.19-7.22$ (m, 1H), 7.28-7.31 (m, 2H), 7.397.41 (m, 2H); ${ }^{13} \mathrm{C}$ NMR (75 MHz) 829.0, 32.0, 62.2, 126.1, 127.1, 128.5, 129.1, 130.3, 131.0, 134.6, 137.5; (4E,6E)-7-Phenyl-4,6-heptadien-1-ol $\mathrm{R}_{f} 0.36$ (2:3 ethyl acetate/hexanes). ${ }^{1} \mathrm{H}$ NMR (600 MHz) $\delta 1.68$ (quin, $\left.J=6.6 \mathrm{~Hz}, 2 \mathrm{H}\right), 2.20-2.24$ (m, $3 \mathrm{H}), 3.63(\mathrm{t}, J=6.6 \mathrm{~Hz}, 2 \mathrm{H}), 5.81(\mathrm{dt}, J=15.0,7.2 \mathrm{~Hz}, 1 \mathrm{H}), 6.22(\mathrm{ddd}, J=15.0,10.2$, $0.6 \mathrm{~Hz}, 1 \mathrm{H}), 6.43(\mathrm{~d}, J=15.6 \mathrm{~Hz}, 1 \mathrm{H}), 6.73(\mathrm{dd}, J=15.6,10.2 \mathrm{~Hz}, 1 \mathrm{H}), 7.16-7.19(\mathrm{~m}$, 1H), 7.26-7.29 (m, 2H), 7.34-7.36 (m, 2H); ${ }^{13} \mathrm{C}$ NMR (75 MHz) $\delta$ 29.0, 32.1, 62.0, 126.0, 127.0, 128.4, 129.1, 130.2, 130.9, 134.6, 137.4; Analysis of mixture IR (neat) 3352, 1668, 1645, 1625, 1596, 1569, 1493, 1448, 1057, $988 \mathrm{~cm}^{-1}$; EIMS m/z: 188 (M+ 64), 170 (6), 169 (8), 155 (34), 141 (48), 128 (100), 115 (61), 104 (27), 91 (86), 77 (34). HRMS (EI) calcd for $\mathrm{C}_{13} \mathrm{H}_{16} \mathrm{O}$ 188.1201, found 188.1206.

2-Methyl-8-phenyl-5,7-octadien-2-ol (5b) Yield: 0.92 g, 52\%: Obtained as a 7:13 mixture of 5Z,7E : 5E,7E isomers. (5Z,7E)-2-Methyl-8-phenyl-5,7-octadien-2-ol Pale yellow oil; $\mathrm{R}_{f} 0.23$ (1:4 ethyl acetate/hexanes); IR (neat) 3391, 1594, 1574, 1492, 1376, 1148, $984 \mathrm{~cm}^{-1} ;{ }^{1} \mathrm{H}$ NMR (300 MHz) $\delta 1.28$ (s, 6H), 1.47 (bs, 1H), 1.60-1.63 (m, 2H), 2.37-2.42 (m, 2H), $5.40(\mathrm{dt}, J=10.8,7.8 \mathrm{~Hz}, 1 \mathrm{H}), 6.14-6.19(\mathrm{~m}, 1 \mathrm{H}), 6.40$ (d, $J=15.6 \mathrm{~Hz}, 1 \mathrm{H}), 7.07$ (ddd, $J=15.6,11.4,1.2 \mathrm{~Hz}, 1 \mathrm{H}), 7.20-7.23(\mathrm{~m}, 1 \mathrm{H}), 7.30-$ 7.33 (m, 2H), 7.40-7.42 (m, 2H); ${ }^{13} \mathrm{C}$ NMR (75 MHz) $\delta 23.0,29.3,43.5,70.9,124.1$, 126.3, 127.4, 128.5, 128.8, 132.3, 132.7, 137.5; (5E,7E)-2-Methyl-8-phenyl-5,7octadien-2-ol Pale yellow crystals; $\mathrm{R}_{f} 0.16$ (1:4 ethyl acetate/hexanes); IR (nujol) 3272, 1594, 1575, 1463, 1376, 1206, $985 \mathrm{~cm}^{-1} ;{ }^{1} \mathrm{H}$ NMR (300 MHz) $\delta 1.25(\mathrm{~s}, 6 \mathrm{H})$, 1.45 (bs, 1H), 1.60-1.63 (m, 2H), 2.23-2.27 (m, 2H), $5.85(\mathrm{dt}, J=13.8,7.2 \mathrm{~Hz}, 1 \mathrm{H})$, 
6.22-6.27 (m, 1H), $6.45(\mathrm{~d}, J=15.6 \mathrm{~Hz}, 1 \mathrm{H}), 6.74(\mathrm{dd}, J=15.6,10.2 \mathrm{~Hz}, 1 \mathrm{H}), 7.18-$ $7.21(\mathrm{~m}, 1 \mathrm{H}), 7.28-7.31(\mathrm{~m}, 2 \mathrm{H}), 7.36-7.38(\mathrm{~m}, 2 \mathrm{H}) ;{ }^{13} \mathrm{C} \mathrm{NMR}(75 \mathrm{MHz}) \delta 27.8,29.3$, 43.1, 70.8, 126.1, 127.1, 128.5, 129.2, 130.2, 130.6, 135.4, 137.6; Analysis of mixture EIMS m/z: $216.2\left(\mathrm{M}^{+}, 25\right), 198.1$ (44), 143.1 (100). HRMS calcd for $\mathrm{C}_{15} \mathrm{H}_{20} \mathrm{O} 216.1514$, found 216.1524.

1,1,7-Triphenyl-4,6-heptadien-1-ol (5c) Yield: 1.52 g, 64\%; Pale yellow oil; 18:82 $4 Z, 6 E^{a}: 4 E, 6 E^{b} ; \mathrm{R}_{f} 0.27$ (1:9 ethyl acetate/hexanes). IR (neat) 3559, 3472, 1667, 1643, 1596, 1493, 1447, $989 \mathrm{~cm}^{-1} ;{ }^{1} \mathrm{H}$ NMR (300 MHz) $\delta$ 2.13-2.19 (m, 3H, includes $\mathrm{OH})^{a, b}, 2.39-2.44(\mathrm{~m}, 3 \mathrm{H} \text {, includes } \mathrm{OH})^{a, b}, 5.54-5.64(\mathrm{~m}, 1 \mathrm{H})^{a}, 5.83(\mathrm{dt}, J=15.6,7.8$ $\mathrm{Hz}, 1 \mathrm{H})^{b}, 6.11-6.22(\mathrm{~m}, 1 \mathrm{H})^{a, b}, 6.42(\mathrm{~d}, J=15.6 \mathrm{~Hz}, 1 \mathrm{H})^{b}, 6.48(\mathrm{~d}, J=15.6 \mathrm{~Hz}, 1 \mathrm{H})^{a}$, $6.72(\mathrm{dd}, J=15.6,10.8 \mathrm{~Hz}, 1 \mathrm{H})^{b}, 6.79(\mathrm{ddd}, J=15.6,10.8,1.2 \mathrm{~Hz}, 1 \mathrm{H})^{a}, 7.16-7.46$ $(\mathrm{m}, 15 \mathrm{H}){ }^{a, b} ;{ }^{13} \mathrm{C}$ NMR $(75 \mathrm{MHz}) \delta 22.9^{a}, 27.4^{b}, 41.4^{b}, 41.9^{a}, 78.2^{b}, 78.4^{a}, 125.7$, $126.0,126.2,126.3,126.4,126.9,127.1,127.4,127.8,128.0,128.19,128.23,128.49$, 128.52, 129.2, 129.4, 130.4, 130.9, 132.3, 132.5, 135.0, 137.6, 146.8; EIMS m/z: 340 $\left(\mathrm{M}^{+}, 17\right), 322$ (13), 281 (10), 231 (38), 205 (19), 180 (40), 165 (18), 142 (12), 128 (19), 115 (23), 105 (100). HRMS calcd for $\mathrm{C}_{25} \mathrm{H}_{24} \mathrm{O} 340.1827$, found 340.1830.

8-Phenyl-5,7-octadien-2-ol (5d) Yield: $6.24 \mathrm{~g}$, 53\%; Colourless oil; Obtained as a mixture of 3:7 5Z,7E : 5E,7E isomers. (5Z,7E)-8-Phenyl-5,7-octadien-2-ol $\mathrm{R}_{f} 0.30$ (2:3 ethyl acetate/hexanes). ${ }^{1} \mathrm{H}$ NMR $(600 \mathrm{MHz}) \delta 1.20(\mathrm{~d}, J=6.0 \mathrm{~Hz}, 3 \mathrm{H}), 1.51-1.62$ (m, 2H), 1.75 (br s, 1H), 2.36-2.40 (m, 2H), 3.81-3.86 (m, 1H), $5.51(\mathrm{dt}, J=10.8,7.8$ $\mathrm{Hz}, 1 \mathrm{H}), 1.67(\mathrm{ddd}, J=10.8,10.8,1.2 \mathrm{~Hz}, 1 \mathrm{H}), 6.52$ (d, $J=15.6 \mathrm{~Hz}, 1 \mathrm{H}), 7.08$ (ddd, $J=15.6,10.8,1.2 \mathrm{~Hz}, 1 \mathrm{H}), 7.19-7.21(\mathrm{~m}, 1 \mathrm{H}), 7.28-7.31(\mathrm{~m}, 2 \mathrm{H}), 7.39-7.41(\mathrm{~m}, 2 \mathrm{H})$; ${ }^{13} \mathrm{C}$ NMR $(75 \mathrm{MHz}) \delta 23.6,24.4,38.9,67.5,124.1,126.3,127.4,128.6,129.2,132.2$, 


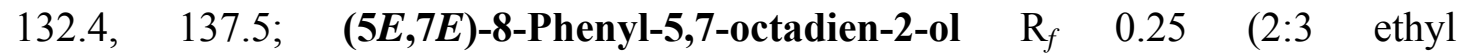
acetate/hexanes). ${ }^{1} \mathrm{H}$ NMR (600 MHz) $\delta 1.19(\mathrm{~d}, J=6.0 \mathrm{~Hz}, 3 \mathrm{H}), 1.51-1.61(\mathrm{~m}, 2 \mathrm{H})$, 1.87 (br s, 1H), 2.18-2.27 (m, 2H), 3.80 (sextet, $J=6.0 \mathrm{~Hz}, 1 \mathrm{H}), 5.81(\mathrm{dt}, J=15.0$, $7.2 \mathrm{~Hz}, 1 \mathrm{H}), 6.22$ (ddd, $J=15.0,10.8,1.2 \mathrm{~Hz}, 1 \mathrm{H}), 6.43$ (d, $J=15.6 \mathrm{~Hz}, 1 \mathrm{H}), 6.73$ (dd, $J=15.6,10.8 \mathrm{~Hz}, 1 \mathrm{H}), 7.16-7.19(\mathrm{~m}, 1 \mathrm{H}), 7.26-7.29(\mathrm{~m}, 2 \mathrm{H}), 7.34-7.36(\mathrm{~m}, 2 \mathrm{H})$; ${ }^{13} \mathrm{C}$ NMR $(75 \mathrm{MHz}) \delta 23.5,29.2,38.6,67.6,126.1,127.1,128.5,129.1,130.3,130.9$, 134.9, 137.5; Analysis of mixture IR (neat) 3369, 1594, 1574, 1448, 1374, 1126, 988 $\mathrm{cm}^{-1}$; EIMS m/z $202\left(\mathrm{M}^{+}, 76\right), 169$ (16), 155 (100), 132 (67), 129 (78), 104 (39), 91 (85); HRMS calcd for $\mathrm{C}_{14} \mathrm{H}_{18} \mathrm{O} 202.1358$, found 202.1357.

\section{SCHEME 1.}<smiles>[R17]C(=O)C=[Pb]</smiles>

$$
\text { 28a: } R^{1}=E t
$$$$
\text { 28b: } R^{1}=M e
$$$$
\mathrm{R}^{2} \stackrel{+}{\mathrm{\curvearrowright}}
$$

29a: $\mathrm{R}^{2}=p-\mathrm{MeoPh}$

29b: $\mathrm{R}^{2}=p-\mathrm{BrPh}$

29c: $R^{2}=$ naphthyl

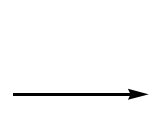

30a: $\mathrm{R}^{1}=\mathrm{Et}, \mathrm{R}^{2}=p-\mathrm{MeoPh}$

30b: $\mathrm{R}^{1}=\mathrm{Et}, \mathrm{R}^{2}=p-\mathrm{BrPh}$

30c: $R^{1}=M e, R^{2}=$ naphthyl

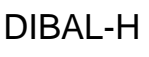

31a-c
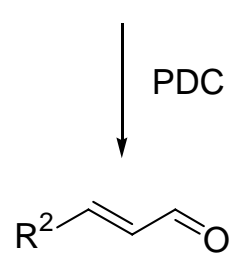

6a-c

General procedure for formation of $\boldsymbol{\alpha}, \boldsymbol{\beta}$-unsaturated esters 30a-c. ${ }^{1}$ Ethyl (triphenylphosphoranylidene)acetate 28a or methyl (triphenylphosphoranylidene)acetate $\mathbf{2 8 b}$ (1.05 equiv) was added to a solution of aldehyde 29a-c (1 equiv) in anhydrous dichloromethane $(10 \mathrm{~mL} / \mathrm{g})$ under an inert atmosphere $\left(\mathrm{N}_{2}\right)$. The solution was stirred for 16 hours at $22^{\circ} \mathrm{C}$. Volatiles were removed in vacuo and the resulting solid purified by flash chromatography to give the desired $\alpha, \beta$-unsaturated ester 30a-c. 
Ethyl (E)-3-(4-methoxyphenyl)-2-propenoate $\quad$ (30a) $^{2} \quad$ Yield: 8.13 g, 100\%; Colourless solid; $\mathrm{R}_{f} 0.60$ (1:4 ethyl acetate/hexanes); ${ }^{1} \mathrm{H}$ NMR $(300 \mathrm{MHz}) \delta 1.33(\mathrm{t}, J$ $=7.2 \mathrm{~Hz}, 3 \mathrm{H}), 3.83(\mathrm{~s}, 3 \mathrm{H}), 4.25(\mathrm{q}, J=7.2 \mathrm{~Hz}, 2 \mathrm{H}), 6.31(\mathrm{~d}, J=15.9 \mathrm{~Hz}, 1 \mathrm{H}), 6.90$ (d, $J=6.9 \mathrm{~Hz}, 2 \mathrm{H}), 7.47(\mathrm{~d}, J=6.9 \mathrm{~Hz}, 2 \mathrm{H}), 7.64(\mathrm{~d}, J=15.9 \mathrm{~Hz}, 1 \mathrm{H})$.

Ethyl (E)-3-(4-bromophenyl)-2-propenoate $\quad(30 b)^{3} \quad$ Yield: 10.68 g, 100\%; Colourless oil; ${ }^{1} \mathrm{H}$ NMR (300 MHz) $\delta 1.27(\mathrm{t}, J=6.9 \mathrm{~Hz}, 3 \mathrm{H}), 4.22(\mathrm{q}, J=6.9 \mathrm{~Hz}$, 2H), $6.32(\mathrm{~d}, J=15.9 \mathrm{~Hz}, 1 \mathrm{H}), 7.33-7.35(\mathrm{~m}, 4 \mathrm{H}), 7.59$, (d, J = 15.9 Hz, 1H).

Methyl (E)-3-(2-naphthyl)-2-propenoate (30c) ${ }^{1}$ Yield: 6.80 g, 99\%; Colourless solid; $\mathrm{R}_{f} 0.77$ (2:3 ethyl acetate/hexanes); ${ }^{1} \mathrm{H}$ NMR (300 MHz) $\delta 3.83$ (s, 3H), 6.55 (d, $J=15.9 \mathrm{~Hz}, 1 \mathrm{H}), 7.48-7.54(\mathrm{~m}, 2 \mathrm{H}), 7.66(\mathrm{dd}, J=8.4,1.8 \mathrm{~Hz}, 1 \mathrm{H}), 7.82-7.89(\mathrm{~m}$, 4H), 7.92 (br s, 1H).

General procedure for formation of $\boldsymbol{\alpha}, \boldsymbol{\beta}$-unsaturated alcohols $31 \mathrm{a}-\mathrm{c}^{1}{ }^{1}$ To a cooled solution $\left(-78^{\circ} \mathrm{C}\right)$ of $\alpha, \beta$-unsaturated ester 30a-c (1 equiv) in anhydrous dichloromethane under an inert atmosphere was added dropwise a $1.5 \mathrm{M}$ solution of DIBAL-H in toluene (2.1 equiv) over 30-60 minutes. The reaction was quenched after stirring for 90 minutes at $-78^{\circ} \mathrm{C}$ by the slow addition of $10 \%$ aqueous $\mathrm{NaOH}$ solution at this temperature. The solution was warmed to $22^{\circ} \mathrm{C}$ and the organic phase separated. The aqueous phase was further extracted with dichloromethane. The combined organic phases were dried $\left(\mathrm{MgSO}_{4}\right)$, filtered and concentrated in vacuo to give the desired alcohol 31a-c. 
(E)-3-(4-Methoxyphenyl)-2-propen-1-ol (31a) ${ }^{1}$ Yield: 6.06 g, 94\%; Colourless solid; $\mathrm{R}_{f} 0.50$ (2:3 ethyl acetate/hexanes); ${ }^{1} \mathrm{H}$ NMR (300 MHz) $\delta 3.80$ (s, 3H), 4.28 (d, $J=5.7 \mathrm{~Hz}, 2 \mathrm{H}), 4.60($ br s, $1 \mathrm{H}), 6.22(\mathrm{dt}, J=15.3,5.7 \mathrm{~Hz}, 1 \mathrm{H}), 6.54(\mathrm{~d}, J=15.3 \mathrm{~Hz}$, 1H), 6.83-6.91 (m, 2H), 7.26-7.34 (m, 2H).

(E)-3-(4-Bromophenyl)-2-propen-1-ol (31b) ${ }^{4}$ Yield: 8.23 g, 98\%; Colourless solid; ${ }^{1} \mathrm{H}$ NMR (300 MHz) $\delta 2.40($ br s, $1 \mathrm{H}), 4.16(\mathrm{~d}, J=5.7 \mathrm{~Hz}, 2 \mathrm{H}), 6.13(\mathrm{dt}, J=$ 15.3, $5.7 \mathrm{~Hz}, 1 \mathrm{H}), 6.42,(\mathrm{~d}, J=15.3 \mathrm{~Hz}, 1 \mathrm{H}), 7.03,(\mathrm{~d}, J=6.9 \mathrm{~Hz}, 2 \mathrm{H}), 7.24(\mathrm{~d}, J=$ $6.9 \mathrm{~Hz}, 2 \mathrm{H})$.

(E)-3-(2-Naphthyl)-2-propen-1-ol (31c) ${ }^{1}$ Yield: 5.26 g, 97\%; Colourless solid; $\mathrm{R}_{f}$ 0.38 (2:3 ethyl acetate/hexanes). ${ }^{1} \mathrm{H}$ NMR (200 MHz) $\delta 1.59$ (br s, 1H), 4.38 (t, $J=$ $5.6 \mathrm{~Hz}, 2 \mathrm{H}), 6.49(\mathrm{dt}, J=16.0,5.6 \mathrm{~Hz}, 1 \mathrm{H}), 6.79(\mathrm{~d}, J=16.0 \mathrm{~Hz}, 1 \mathrm{H}), 7.42-7.50(\mathrm{~m}$, 2H), $7.60(\mathrm{dd}, J=8.6,1.8 \mathrm{~Hz}, 1 \mathrm{H}), 7.73-7.83(\mathrm{~m}, 4 \mathrm{H})$.

General procedure for formation of $\boldsymbol{\alpha}, \boldsymbol{\beta}$-unsaturated aldehydes $6 \mathrm{a}-\mathrm{c}^{5} .^{5}$ To a solution of $\alpha, \beta$-unsaturated alcohol 31a-c (1 equiv) in anhydrous dichloromethane was added PDC (1.5 equiv). After stirring for 24 hours at $22^{\circ} \mathrm{C}$, the solution was diluted by half with diethyl ether and hexane $(1: 1)$ and filtered through silica $(5 \mathrm{~cm})$. The silica was washed with 2:3 ethyl acetate/hexanes until all aldehyde had been removed from the silica.. The combined filtrate was concentrated in vacuo to give the desired aldehyde 6a-c. The aldehyde was used without further purification.

(E)-3-(4-Methoxyphenyl)-2-propenal (6a) ${ }^{6}$ Yield: 3.80 g, 63\%; Pale yellow oil; ${ }^{1} \mathrm{H}$ NMR (300 MHz) $\delta 3.86(\mathrm{~s}, 3 \mathrm{H}), 4.11(\mathrm{q}, J=7.2 \mathrm{~Hz}, 2 \mathrm{H}), 6.61(\mathrm{dd}, J=15.9,7.8$ 
$\mathrm{Hz}, 1 \mathrm{H}), 6.92-6.97(\mathrm{~m}, 2 \mathrm{H}), 7.43(\mathrm{~d}, J=15.9 \mathrm{~Hz}, 1 \mathrm{H}), 7.50-7.55(\mathrm{~m}, 2 \mathrm{H}), 9.65(\mathrm{~d}, J=$ $7.8 \mathrm{~Hz}, 1 \mathrm{H})$.

(E)-3-(4-Bromophenyl)-2-propenal (6b) ${ }^{7}$ Yield: 6.64 g, 82\%; Colourless solid; ${ }^{1} \mathrm{H}$ NMR (300 MHz) $\delta 6.71(\mathrm{dd}, J=16.2,7.5 \mathrm{~Hz}, 1 \mathrm{H}), 7.40-7.46(\mathrm{~m}, 3 \mathrm{H}), 7.55-7.59(\mathrm{~m}$, 2H), $9.71(\mathrm{~d}, J=7.5 \mathrm{~Hz}, 1 \mathrm{H})$.

(E)-3-(2-Naphthyl)-2-propenal (6c) ${ }^{8}$ Yield: 2.36 g, 92\%; Pale yellow plates; $\mathrm{R}_{f}$ 0.42 (1:4 ethyl acetate/hexanes). ${ }^{1} \mathrm{H}$ NMR $(300 \mathrm{MHz}) \delta 6.80(\mathrm{dd}, J=15.6,7.8 \mathrm{~Hz}$, 1H), 7.51-7.57 (m, 2H), $7.64(\mathrm{dd}, J=8.7,1.8 \mathrm{~Hz}, 1 \mathrm{H}), 7.81-7.88(\mathrm{~m}, 4 \mathrm{H}), 7.94(\mathrm{br} \mathrm{s}$, 1H), $9.73(\mathrm{~d}, J=7.8 \mathrm{~Hz}, 1 \mathrm{H}) ;{ }^{13} \mathrm{C}$ NMR $(300 \mathrm{MHz}) \delta 123.5,126.9,127.7,127.8$, $128.6,128.7,128.9,130.6,131.5,133.1,134.6,152.6,193.5$.

\section{4-\{[1-(tert-Butyl)-1,1-dimethylsilyl]oxy\}butyl)(triphenyl)phosphonium iodide}

(7) $^{9} \quad 1-\left[\left(\right.\right.$ tert-butyldimethylsilyl)oxy]-4-iodobutane ${ }^{10}(20.4 \quad \mathrm{~g}, \quad 64.9 \mathrm{mmol})$ and triphenylphosphine $(22.13 \mathrm{~g}, 84.4 \mathrm{mmol})$ were melted together at $90^{\circ} \mathrm{C}$ for 3 hours. Diethyl ether $(100 \mathrm{~mL})$ was added to the cooled solution. The white precipitate was broken up into a fine powder and stirred for 2 hours prior to collection by vacuum filtration. The powder was washed with diethyl ether $(3 \times 100 \mathrm{~mL})$ and dried in vacuo. Yield: $31.42 \mathrm{~g}, 84 \%$; Colourless solid.

General procedure for formation of protected dienes 8. To a cooled suspension $\left(-10\right.$ to $\left.-15^{\circ} \mathrm{C}\right)$ of ylide salt 7 (1.05 equiv) in anhydrous THF $(10 \mathrm{~mL} / \mathrm{g}$ of 7$)$ was added drop-wise and with stirring a solution of $2.3 \mathrm{M} n$-BuLi in hexanes (1.05 equiv). After stirring for 30 minutes a solution of aldehyde $\mathbf{6 a - c}$ (1 equiv) in anhydrous THF 
$\left(10 \mathrm{~mL} / \mathrm{g}\right.$ of aldehyde) was added at $-10^{\circ} \mathrm{C}$. After stirring for 16 hours at $22^{\circ} \mathrm{C}$, protected from light, the solution was diluted by half with hexanes and filtered through silica $(3 \mathrm{~cm})$. The silica was washed with 2:3 ethyl acetate/hexanes until all diene had been removed and the combined filtrates concentrated in vacuo. The residue obtained was further purified by flash chromatography to give an inseparable mixture of $Z, E$ and $E, E$ dienes 8a-c, the hydroxyl derivatives, of which, were fully characterised.

tert-Butyl[7-(4-methoxyphenyl)-4.6-hepta-dienyl)oxy] dimethylsilane

Yield: 2.78 g, 79\%; Colourless solid; $\mathrm{R}_{f} 0.69$ (1:19 ethyl acetate/hexanes);

[(7-(4-Bromophenyl)-4,6-heptadienyl)oxy](tert-butyl)dimethylsilane (8b) Yield: 6.96 g, 86\%; Colourless oil; $\mathrm{R}_{\mathrm{f}} 0.65$ (1:19 ethyl acetate/hexanes).

[(7-(2-Naphthyl)-4,6-heptadienyl)oxy](tert-butyl)dimethylsilane (8c) Yield: 2.11 g, 70\%; Yellow solid; 47:53 4Z,6E : 4E,6E; $\mathrm{R}_{f} 0.42$ (1:4 ethyl acetate/hexanes).

General procedure for TBDMS cleavage: Synthesis of 9a-c. ${ }^{11}$ To a solution of protected diene 8a-c (1 equiv) in anhydrous THF (10 mL / $\mathrm{g}$ of 8) was added a 1.0M solution of tetrabutylammonium fluoride in THF (1.5 equiv). The solution was stirred for at $22^{\circ} \mathrm{C}$ protected from light until t.l.c. analysis (1:9 ethyl acetate:hexanes) indicated that all protected diene had been consumed (4-16 hours). The solution was concentrated in vacuo and the resulting residue purified by flash chromatography to give a mixture of $Z, E$ and $E, E$-hydroxy dienes 9a-c. 
7-(4-Methoxyphenyl)-4,6-heptadien-1-ol (9a) Yield: 1.78 g, 98\%; Colourless oil; Obtained as a 2:3 mixture of 4Z,6E : 4E,6E isomers. (4Z,6E)-7-(4-Methoxyphenyl)4,6-heptadien-1-ol $\mathrm{R}_{f} 0.41$ (7:13 ethyl acetate/hexanes); ${ }^{1} \mathrm{H}$ NMR (300 MHz) $\delta 1.65$ $1.74(\mathrm{~m}, 3 \mathrm{H}), 2.33-2.42(\mathrm{~m}, 2 \mathrm{H}), 3.69(\mathrm{t}, J=6.6 \mathrm{~Hz}, 2 \mathrm{H}), 3.80(\mathrm{~s}, 3 \mathrm{H}), 5.47(\mathrm{dt}, J=$ 10.8, 7.8 Hz, 1H), 6.13-6.20 (m, 1H), 6.48 (d, $J=15.6 \mathrm{~Hz}, 1 \mathrm{H}), 6.82-6.87(\mathrm{~m}, 2 \mathrm{H})$, 6.95 (ddd, $J=15.6,10.8,1.2 \mathrm{~Hz}, 1 \mathrm{H}), 7.32-7.37(\mathrm{~m}, 2 \mathrm{H}) ;{ }^{13} \mathrm{C}$ NMR $(75 \mathrm{MHz}) \delta$ $24.2,32.4,55.2,62.2,114.0,122.6,127.5,129.5,130.3,130.8,131.9,159.1$; (4E,6E)-7-(4-Methoxyphenyl)-4,6-heptadien-1-ol $\quad \mathrm{R}_{f} \quad 0.39 \quad(7: 13 \quad$ ethyl acetate/hexanes); ${ }^{1} \mathrm{H}$ NMR (300 MHz) $\delta 1.57$ (br s, OH), 1.57-1.67 (m, 2H), 2.16-2.27 (m, 2H), $3.67(\mathrm{t}, J=6.6 \mathrm{~Hz}, 2 \mathrm{H}), 3.79(\mathrm{~s}, 3 \mathrm{H}), 5.77(\mathrm{dt}, J=15.3,7.8 \mathrm{~Hz}, 1 \mathrm{H}), 6.17-$ $6.26(\mathrm{~m}, 1 \mathrm{H}), 6.40(\mathrm{~d}, J=15.6 \mathrm{~Hz}, 1 \mathrm{H}), 6.95(\mathrm{dd}, J=15.6,10.5 \mathrm{~Hz}, 1 \mathrm{H}) 6.81-6.89$

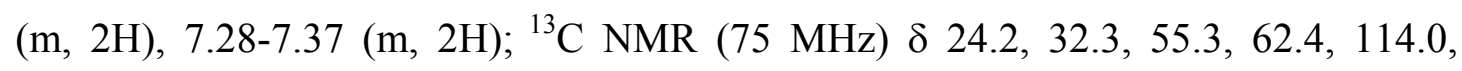
127.2, 127.3, 130.0, 130.4, 131.2, 133.4, 159.0; Analysis of mixture IR (neat) 3302, 1602, 1511, 1252, 1028, $986 \mathrm{~cm}^{-1}$; EIMS m/z: $234\left(\left[\mathrm{M}+\mathrm{H}_{2} \mathrm{O}\right]^{+}, 16\right), 218\left(\mathrm{M}^{+}, 100\right)$, 187 (6), 173 (30), 163 (28), 147 (35), 121 (41), 108 (10), 91 (18), 71 (23), 43 (12); HRMS (EI) calcd for $\mathrm{C}_{14} \mathrm{H}_{18} \mathrm{O}_{2} 218.1307$, found 218.1302 .

7-(4-Bromophenyl)-4,6-heptadien-1-ol (9b) Yield: 2.46 g, 50\%; Pale yellow solid; m.p. $90{ }^{\circ} \mathrm{C}$; Obtained as a $2: 3$ mixture of $4 Z, 6 E: 4 E, 6 E$ isomers. (4Z,6E)-7-(4Bromophenyl)-4,6-heptadien-1-ol $\mathrm{R}_{f} 0.71$ (1:1 ethyl acetate/hexanes); ${ }^{1} \mathrm{H}$ NMR (300 MHz) $\delta 1.64-1.74(\mathrm{~m}, 2 \mathrm{H}), 1.98(\mathrm{br} \mathrm{s}, \mathrm{OH}), 2.33-2.41(\mathrm{~m}, 2 \mathrm{H}), 3.65(\mathrm{t}, J=6.6 \mathrm{~Hz}$, 2H), $5.55(\mathrm{dt}, J=10.5,7.5 \mathrm{~Hz}, 1 \mathrm{H}), 6.12-6.24(\mathrm{~m}, 1 \mathrm{H}), 6.44(\mathrm{~d}, J=15.9 \mathrm{~Hz}, 1 \mathrm{H})$, $7.05(\mathrm{ddd}, J=15.9,11.1,1.2 \mathrm{~Hz}, 1 \mathrm{H}), 7.18-7.27(\mathrm{~m}, 2 \mathrm{H}), 7.37-7.43(\mathrm{~m}, 2 \mathrm{H}) ;{ }^{13} \mathrm{C}$ NMR (75 MHz) $\delta 24.3,32.3,62.1,121.0,124.8,127.8,129.1,131.0,131.6,132.7$, 136.42; EIMS m/z: $268\left([\mathrm{M}+\mathrm{H}]^{+}, 100\right), 266(100), 221$ (17), 182 (11), 169 (51), 142 
(74), 128 (46), 91 (19), 84 (33), 51 (12), 39 (21) (4E,6E)-7-(4-Bromophenyl)-4,6heptadien-1-ol $\mathrm{R}_{f} 0.61$ (1:1 ethyl acetate/hexanes); ${ }^{1} \mathrm{H}$ NMR $(300 \mathrm{MHz}) \delta 1.50$ (br s, $\mathrm{OH}), 1.66-1.75(\mathrm{~m}, 2 \mathrm{H}), 2.21-2.28(\mathrm{~m}, 2 \mathrm{H}), 3.68(\mathrm{t}, J=6.6 \mathrm{~Hz}, 2 \mathrm{H}), 5.85(\mathrm{dt}, J=$ 15.0, 7.8 Hz, 1H), 6.18-6.26 (m, 1H), 6.37 (d, $J=15.6 \mathrm{~Hz}, 1 \mathrm{H}), 6.73$ (dd, $J=15.6$, $7.5 \mathrm{~Hz}, 1 \mathrm{H}), 7.20-7.25(\mathrm{~m}, 2 \mathrm{H}), 7.39-7.43(\mathrm{~m}, 2 \mathrm{H}) ;{ }^{13} \mathrm{C} \mathrm{NMR}(75 \mathrm{MHz})(4 E, 6 E) \delta$ 29.1, 32.1, 62.3, 120.8, 127.6, 129.1, 129.9, 130.8, 131.6, 135.5, 136.5; EIMS m/z 268 $\left([\mathrm{M}+\mathrm{H}]^{+}, 54\right), 266(55), 221(20), 197(4), 182(14), 169$ (68), 142 (100), 128 (60), 91 (20), 84 (28), 51 (14), 39 (23);. Analysis of mixture IR (nujol) 3307, 1643, 1624 , 1585, 1072, 1008, 995, $826 \mathrm{~cm}^{-1}$; HRMS (EI) calcd for $\mathrm{C}_{13} \mathrm{H}_{15} \mathrm{OBr} 266.0307$, found 266.0309 .

\section{(4Z,6E)-7-(2-Naphthyl)-4,6-heptadien-1-ol and (4E,6E)-7-(2-naphthyl)-4,6-} heptadien-1-ol (9c) Yield: $1.22 \mathrm{~g}, 88 \%$; Pale yellow solid; Obtained as a 37:63 mixture of $4 Z, 6 E^{a}: 4 E, 6 E^{b}$ isomers; $\mathrm{R}_{f} 0.68^{a}$ and $0.61^{b}$ (3:2 ethyl acetate/hexanes). IR (nujol) 3325, 1590, 1060, 989, 820, $741 \mathrm{~cm}^{-1}$; ${ }^{1} \mathrm{H}$ NMR (600 MHz) $\delta 1.34$ (br s, $1 \mathrm{H})^{a, b}, 1.70-1.77(\mathrm{~m}, 2 \mathrm{H})^{a, b}, 2.27(\mathrm{dq}, J=7.8,1.8 \mathrm{~Hz}, 2 \mathrm{H})^{b}, 2.44(\mathrm{dq}, J=7.8,1.8 \mathrm{~Hz}$, $2 \mathrm{H})^{a}, 3.70(\mathrm{t}, J=6.6 \mathrm{~Hz}, 1 \mathrm{H})^{b}, 3.72(\mathrm{t}, J=6.6 \mathrm{~Hz}, 1 \mathrm{H})^{a}, 5.57(\mathrm{dt}, J=10.8,7.8 \mathrm{~Hz}$, $1 \mathrm{H})^{a}, 5.88(\mathrm{dt}, J=15.6,7.8 \mathrm{~Hz}, 1 \mathrm{H})^{b}, 6.23-6.32(\mathrm{~m}, 1 \mathrm{H})^{a, b}, 6.61(\mathrm{~d}, J=15.6 \mathrm{~Hz}, 1 \mathrm{H})^{b}$, $6.70(\mathrm{~d}, J=15.6 \mathrm{~Hz}, 1 \mathrm{H})^{a}, 6.87(\mathrm{dd}, J=15.6,10.2 \mathrm{~Hz}, 1 \mathrm{H})^{b}, 7.20(\mathrm{ddd}, J=15.6,11.4$, $1.8 \mathrm{~Hz}, 1 \mathrm{H})^{a}, 7.39-7.46(\mathrm{~m}, 2 \mathrm{H})^{a, b}, 7.59-7.66(\mathrm{~m}, 1 \mathrm{H})^{a, b}, 7.70-7.80(\mathrm{~m}, 4 \mathrm{H})^{a, b} ;{ }^{13} \mathrm{C}$ NMR $(150 \mathrm{MHz}) \delta 24.3,29.1,32.1,32.4,62.1,62.2,123.4,123.5,124.5,125.6$, $125.7,125.9,126.1,126.2,127.6,127.8,127.9,128.1,128.1,129.4,129.5,130.4$, 131.1, 132.2, 132.5, 132.8, 132.9, 133.6, 134.9, 135.0, 4 masked carbons; EIMS m/z: $238\left(\mathrm{M}^{+}, 94\right), 219$ (11), 191 (43), 179 (100), 165 (29). HMRS calcd for $\mathrm{C}_{17} \mathrm{H}_{18} \mathrm{O}$ 238.1358, found 238.1356. 
General procedure for the synthesis of 1,2-dioxines 10a-g. ${ }^{12}$ A solution of 1,3butadiene (5a-d or 9a-c) in $\mathrm{CH}_{2} \mathrm{Cl}_{2}(30 \mathrm{~mL} / \mathrm{g}$ of $\mathbf{5}$ or 9) was photolysed with $3 \mathrm{x}$ 500W halogen lamps in the presence of Rose Bengal bis(triethylammonium) salt (100 mg) and oxygen for 6 hours. The reaction was performed in a Pyrex flask fitted with an external cooling jacket. The solution was concentrated in vacuo and the resulting residue purified by flash chromatography.

3-(6-Phenyl-3,6-dihydro-1,2-dioxin-3-yl)-1-propanol (10a) Yield: 1.76 g, 43\%; Colourless oil; $\mathrm{R}_{f} 0.27$ (2:3 ethyl acetate/hexanes). IR (neat) 3368, 1599, 1492, 1454 , 1055, $755 \mathrm{~cm}^{-1} ;{ }^{1} \mathrm{H}$ NMR $(300 \mathrm{MHz}) \delta 1.66-1.89(\mathrm{~m}, 5 \mathrm{H}), 3.67(\mathrm{t}, J=6.0 \mathrm{~Hz}, 2 \mathrm{H})$, 4.56-4.61 (m, 1H), 5.53-5.55 (m, 1H), 6.05-6.15 (m, 2H), 7.33-7.40 (m, 5H); ${ }^{13} \mathrm{C}$ NMR $(75 \mathrm{MHz}) \delta 28.8,29.5,62.5,78.3,80.3,126.6,128.51,128.54,128.6,128.8$, 137.4; EIMS m/z: $220\left(\mathrm{M}^{+}, 5\right), 188$ (100), 170 (6), 155 (14), 129 (45), 105 (47), 77 (86). HRMS calcd for $\mathrm{C}_{13} \mathrm{H}_{16} \mathrm{O}_{3} 220.1099$, found 220.1092 .

3-[6-(4-Methoxyphenyl)-3,6-dihydro-1,2-dioxin-3-yl]-1-propanol (10b) Yield: 354 mg, 23\%; Colourless oil; $\mathrm{R}_{f} 0.40$ (1:1 ethyl acetate/hexanes); IR (neat) 3369, $1611,1586,1514,1249,1176,1034 \mathrm{~cm}^{-1} ;{ }^{1} \mathrm{H}$ NMR $(300 \mathrm{MHz}) \delta 1.69-1.85$ (m, 5H), 3.64-3.68 (m, 2H), $3.79(\mathrm{~s}, 3 \mathrm{H}), 4.53-4.56(\mathrm{~m}, 1 \mathrm{H}), 5.49-5.51(\mathrm{~m}, 1 \mathrm{H}), 6.02-6.14(\mathrm{~m}$,

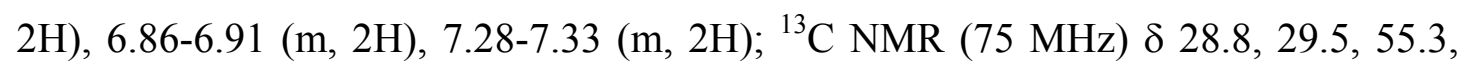
$62.5,78.3,79.9,113.9,126.8,128.5,129.3,130.1,160.1$; EIMS m/z: $250\left(\mathrm{M}^{+}, 16\right)$ 232 (68), 231 (63), 218 (100), 217 (4), 187 (67), 163 (26), 135 (98), 121 (62), 109 (39), 81 (38), 55 (35), 39 (33); HRMS (EI) calcd for $\mathrm{C}_{14} \mathrm{H}_{18} \mathrm{O}_{4}$ 250.1205, found 250.1209 . 
3-[6-(4-Bromophenyl)-3,6-dihydro-1,2-dioxin-3-yl]-1-propanol (10c) Yield: 1.31

g, 54\%; Colourless oil; $\mathrm{R}_{\mathrm{f}} 0.36$ (9:11 ethyl acetate/hexanes). IR (neat) 3381, 1592 , 1486, 1406, 1071, $1012 \mathrm{~cm}^{-1} ;{ }^{1} \mathrm{H}$ NMR $(300 \mathrm{MHz}) \delta 1.69-1.78(\mathrm{~m}, 4 \mathrm{H}), 2.04(\mathrm{br} \mathrm{s}$, $\mathrm{OH}), 3.61-3.67(\mathrm{~m}, 2 \mathrm{H}), 4.55-4.67(\mathrm{~m} .1 \mathrm{H})$, 5.44-5.46 (m, 1H), 6.05-6.11 (m, 2H),

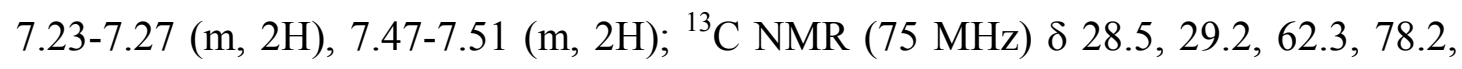

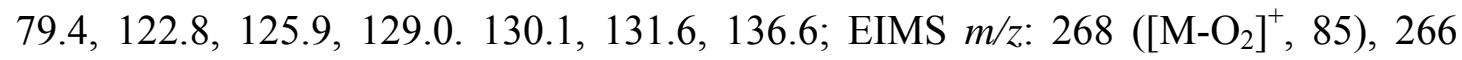
(86), 221 (7), 209 (2), 185 (19), 169 (29), 142 (16), 128 (11), 97 (11), 84 (100), 55 (18), 43 (30); HRMS (EI) calcd for $\mathrm{C}_{13} \mathrm{H}_{15} \mathrm{O}_{3} \mathrm{Br} 298.0205$, found 298.0209.

3-[6-(2-Naphthyl)-3,6-dihydro-1,2-dioxin-3-yl]-1-propanol (10d) Yield: 0.44 g, 39\%; Orange oil; $\mathrm{R}_{f} 0.28$ (2:3 ethyl acetate/hexanes); IR (neat) 3369, 1723, 1693, 1664, 1600, 1508, 1278, 1056, $821 \mathrm{~cm}^{-1} ;{ }^{1} \mathrm{H}$ NMR (300 MHz) $\delta 1.50-1.90(\mathrm{~m}, 4 \mathrm{H})$, 2.54 (br s, $1 \mathrm{H}), 3.55(\mathrm{t}, J=6.0 \mathrm{~Hz}, 2 \mathrm{H}), 4.53-4.56(\mathrm{~m}, 1 \mathrm{H}), 5.63$ (br s, 1H), 6.02-6.11

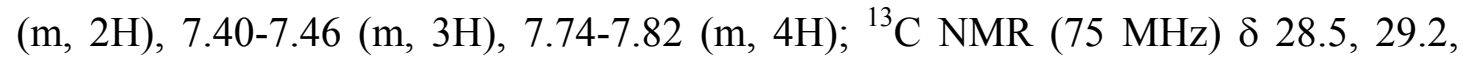
$62.1,78.2,80.1,125.7,126.0,126.2,126.3,127.5,127.8,128.0,128.1,128.6,133.0$, 133.3, 134.8; EIMS m/z: $252\left(\mathrm{M}^{+}-\mathrm{H}_{2} \mathrm{O}, 100\right), 238$ (57), 307 (83).

2-Methyl-4-(6-phenyl-3,6-dihydro-1,2-dioxin-3-yl)-2-butanol (10e). Yield: 0.40 g, 46\%; Colourless oil; $\mathrm{R}_{f} 0.24$ (3:7 ethyl acetate/hexanes). IR (neat) 3401, 1603, 1454, 1378, 1217, 1066, $944 \mathrm{~cm}^{-1} ;{ }^{1} \mathrm{H}$ NMR (300 MHz) $\delta 1.18(\mathrm{~s}, 6 \mathrm{H}), 1.47-1.58(\mathrm{~m}$, 1H), 1.65-1.87 (m, 3H), 1.94 (br s, 1H), 4.50-4.55 (m, 1H), 5.50-5.52 (m, 1H), 6.02$6.13(\mathrm{~m}, 2 \mathrm{H}), 7.32-7.38(\mathrm{~m}, 5 \mathrm{H}) ;{ }^{13} \mathrm{C} \mathrm{NMR}(75 \mathrm{MHz}) \delta 27.8,29.0,29.2,39.2,70.3$, 78.6, 80.1, 126.4, 128.4, 128.4, 128.5, 128.6, 137.4; MS (LSIMS in mnba) m/z: 231.1 ([M-OH] $\left.]^{+}, 100\right), 215.1$ (33), 198.1 (36). HRMS calcd for $\mathrm{C}_{15} \mathrm{H}_{20} \mathrm{O}_{3}-\mathrm{H}_{2} \mathrm{O}$ 230.1307, found 230.1307 . 
1,1-Diphenyl-3-(6-phenyl-3,6-dihydro-1,2-dioxin-3-yl)-1-propanol (10f). Yield: $0.82 \mathrm{~g}, 49 \%$; Pale yellow solid; A small amount was recrystallised from dichloromethane/hexanes; m.p.132-135 $\mathrm{C} . \mathrm{R}_{f} 0.16$ (1:9 ethyl acetate/hexanes). IR (nujol) 3551, 1598, 1336, 1170, 1055, $757 \mathrm{~cm}^{-1} ;{ }^{1} \mathrm{H}$ NMR (300 MHz) $\delta 1.61-1.86(\mathrm{~m}$, 2H), $2.21(\mathrm{~s}, 1 \mathrm{H}), 2.35$ (ddd, $J=14.1,11.1,4.8 \mathrm{~Hz}, 1 \mathrm{H}), 2.57$ (ddd, $J=14.1,11.1,4.8$ $\mathrm{Hz}, 1 \mathrm{H}), 4.52-4.56(\mathrm{~m}, 1 \mathrm{H}), 5.50(\mathrm{br} \mathrm{s}, 1 \mathrm{H}), 5.97-6.05(\mathrm{~m}, 2 \mathrm{H}), 7.17-7.42(\mathrm{~m}, 15 \mathrm{H})$; ${ }^{13} \mathrm{C}$ NMR $(75 \mathrm{MHz}) \delta 27.8,37.4,77.9,78.3,80.2,126.0,126.1,126.7,126.8,126.8$, 128.2, (masked carbon), 128.46, (masked carbon), 128.49, 128.7,137.6, 146.7, 146.9; EIMS m/z: $372\left(\mathrm{M}^{+}, 0.8\right), 354$ (58), 183 (100), 105 (59), 77 (43), 40, (50). HRMS calcd for $\mathrm{C}_{25} \mathrm{H}_{24} \mathrm{O}_{3} 372.1725$, found 372.1735 .

4-(6-Phenyl-3,6-dihydro-1,2-dioxin-3-yl)-2-butanol (10g) Yield: 1.46 g, 70\%; Colourless oil; $\mathrm{R}_{f} 0.4$ (2:3 ethyl acetate/hexanes). IR (neat) 3391, 1602, 1455, 1063, $914 \mathrm{~cm}^{-1} ;{ }^{1} \mathrm{H}$ NMR (300 MHz) $\delta 1.19(\mathrm{~d}, J=6.3 \mathrm{~Hz}, 3 \mathrm{H}), 1.45-1.97$ (m, 5H), 3.77$3.87(\mathrm{~m}, 1 \mathrm{H}), 4.52-4.62,(\mathrm{~m}, 1 \mathrm{H}), 5.52-5.55(\mathrm{~m}, 1 \mathrm{H}), 6.04-6.15(\mathrm{~m}, 2 \mathrm{H}), 7.33-7.40(\mathrm{~m}$, $5 \mathrm{H}) ;{ }^{13} \mathrm{C}$ NMR $(75 \mathrm{MHz}) \delta 23.5,23.6,29.2,29.4,34.9,35.2,67.6,67.8,78.2,78.7$, $80.2,80.3,126.5,126.6,128.49,128.53,128.56$, (masked carbon), 128.6, (masked carbon), 128.7, 128.8, 137.3, 137.4. EIMS m/z: 235 ([M+H] $\left.]^{+}, 9\right), 216$ (4), 202 (100), 184 (9), 155 (29), 133 (25), 117 (15), 105 (36), 81 (60), 45 (35). HRMS calcd for $\mathrm{C}_{14} \mathrm{H}_{18} \mathrm{O}_{3} 234.1256$, found 234.1256. 
General procedure for the synthesis of pyrans. Refer to main paper.

( \pm ) 2-[(2S,3R)-3-Hydroxytetrahydro-2H-2-pyranyl]-1-phenyl-1-ethanone (12a)

colourless solid. m.p. $94-96^{\circ} \mathrm{C} . \mathrm{R}_{f} 0.48$ (3:2 ethyl acetate/hexanes). IR (nujol) 3503, 1669, 1594, 1579, 1096, $690 \mathrm{~cm}^{-1} ;{ }^{1} \mathrm{H}$ NMR $(600 \mathrm{MHz}) \delta 1.46$ (dddd, $J=12.0,12.0$, 12.0, $5.4 \mathrm{~Hz}, 1 \mathrm{H}), 1.66-1.74(\mathrm{~m}, 2 \mathrm{H}), 2.14-2.19(\mathrm{~m}, 1 \mathrm{H}), 2.32(\mathrm{~d}, J=6.0 \mathrm{~Hz}, 1 \mathrm{H})$, $3.24(\mathrm{dd}, J=16.2,7.2 \mathrm{~Hz}, 1 \mathrm{H}), 3.37(\mathrm{ddd}, J=11.4,11.4,3.6 \mathrm{~Hz}, 1 \mathrm{H}), 3.41(\mathrm{dd}, J=$ 16.2, $4.2 \mathrm{~Hz}, 1 \mathrm{H}), 3.40-3.44(\mathrm{~m}, 1 \mathrm{H}), 3.70$ (ddd, $J=9.0,7.2,4.2 \mathrm{~Hz}, 1 \mathrm{H}), 3.85-3.88$ $(\mathrm{m}, 1 \mathrm{H}), 7.44-7.47(\mathrm{~m}, 2 \mathrm{H}), 7.54-7.57(\mathrm{~m}, 1 \mathrm{H}), 7.97-8.00(\mathrm{~m}, 2 \mathrm{H}) ;{ }^{13} \mathrm{C}$ NMR $(150$ MHz) $\delta 25.6,33.2,42.3,67.8,70.7,79.0,128.4,128.5,133.2,137.2,199.6$; EIMS m/z: $221\left(\mathrm{M}^{+}, 82\right), 203$ (100). Anal. Calcd for $\mathrm{C}_{13} \mathrm{H}_{16} \mathrm{O}_{3}: \mathrm{C}, 70.89 ; \mathrm{H}, 7.32$ : found $\mathrm{C}$, $70.92 ; \mathrm{H}, 7.39$.

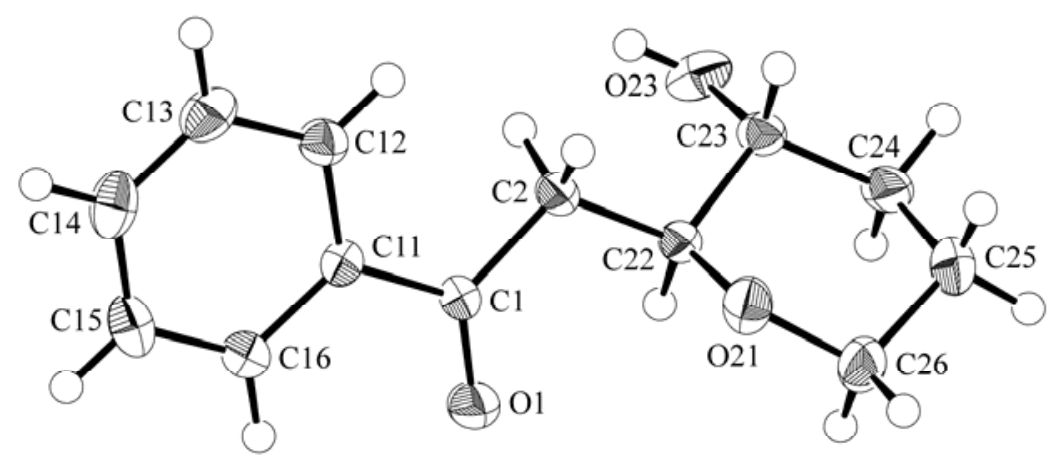

Molecular structure of 12a showing crystallographic numbering scheme employed.

Crystallographic data for 12a: $M=220.3, T=223(2) \mathrm{K}$, orthorhombic, $P 2{ }_{1} 2_{1} 2_{1}, a$ $=5.7086(3), b=13.0589(7), c=15.2878(8) \AA, V=1139.68(10) \AA^{3}, Z=4, D_{\mathrm{x}}=$ 1.284, $F(000)=472, \mu=0.090 \mathrm{~mm}^{-1}$, no. of unique data (Bruker AXS SMART CCD using Mo $\mathrm{K} \alpha$ radiation so that $\left.\theta_{\max }=30.0^{\circ}\right)=3280$, no. of parameters $=145, R$ (all data $)=0.073, w R($ all data $)=0.179, \rho=0.51 \mathrm{e} \AA^{-3}$. The structure was solved by 
direct-methods (SIR92) and refined (anisotropic displacement parameters, $\mathrm{H}$ atoms in the riding model approximation and a weighting scheme $w=1 /\left[\sigma^{2}\left(F_{\mathrm{o}}{ }^{2}\right)+0.0933 P^{2}+\right.$ $0.3462 P]$ where $\left.P=\left(F_{\mathrm{o}}{ }^{2}+2{F_{\mathrm{c}}}^{2}\right) / 3\right)$ with SHELXL-97 on $F^{2}$ using all reflections. The absolute configuration was not determined unambiguously.

( \pm ) 2-[(2S,3S)-3-Hydroxytetrahydro-2H-2-pyranyl]-1-phenyl-1-ethanone (13a)

Colourless oil. $\mathrm{R}_{f} 0.25$ (3:2 ethyl acetate/hexanes). IR (neat) 3446, 1683, 1597, 1580, 1449, 1090, $1000 \mathrm{~cm}^{-1} ;{ }^{1} \mathrm{H}$ NMR (600 MHz) $\delta 1.42-1.46(\mathrm{~m}, 1 \mathrm{H}), 1.76-1.82(\mathrm{~m}, 1 \mathrm{H})$, 1.88-1.94 (m, 1H), 1.96-2.00 (m, 1H), $2.19(\mathrm{~d}, J=9.0 \mathrm{~Hz}, 1 \mathrm{H}), 3.19$ (dd, $J=16.8,6.6$ Hz, 1H), 3.32 (dd, $J=16.8,6.6 \mathrm{~Hz}, 1 \mathrm{H}) 3.55(\mathrm{ddd}, J=12.6,12.0,3.0 \mathrm{~Hz}, 1 \mathrm{H}), 3.75-$ $3.79(\mathrm{~m}, 1 \mathrm{H}), 3.94-3.98(\mathrm{~m}, 1 \mathrm{H}), 4.07(\mathrm{ddd}, J=6.6,6.6,1.2 \mathrm{~Hz}, 1 \mathrm{H}), 7.44-7.47(\mathrm{~m}$,

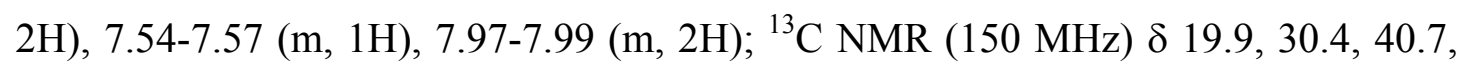
66.6, 68.6, 76.3, 128.2, 128.5, 133.2, 137.1, 198.2; EIMS m/z: $221\left([\mathrm{M}+\mathrm{H}]^{+}, 8\right), 202$ (27), 105 (100), 77 (64), 71 (43). 
$( \pm)(2 S, 3 S)-2-[2-(2-N a p h t h y l)-2-o x o e t h y l]$ tetrahydro-2H-3-pyranyl acetate (16d).

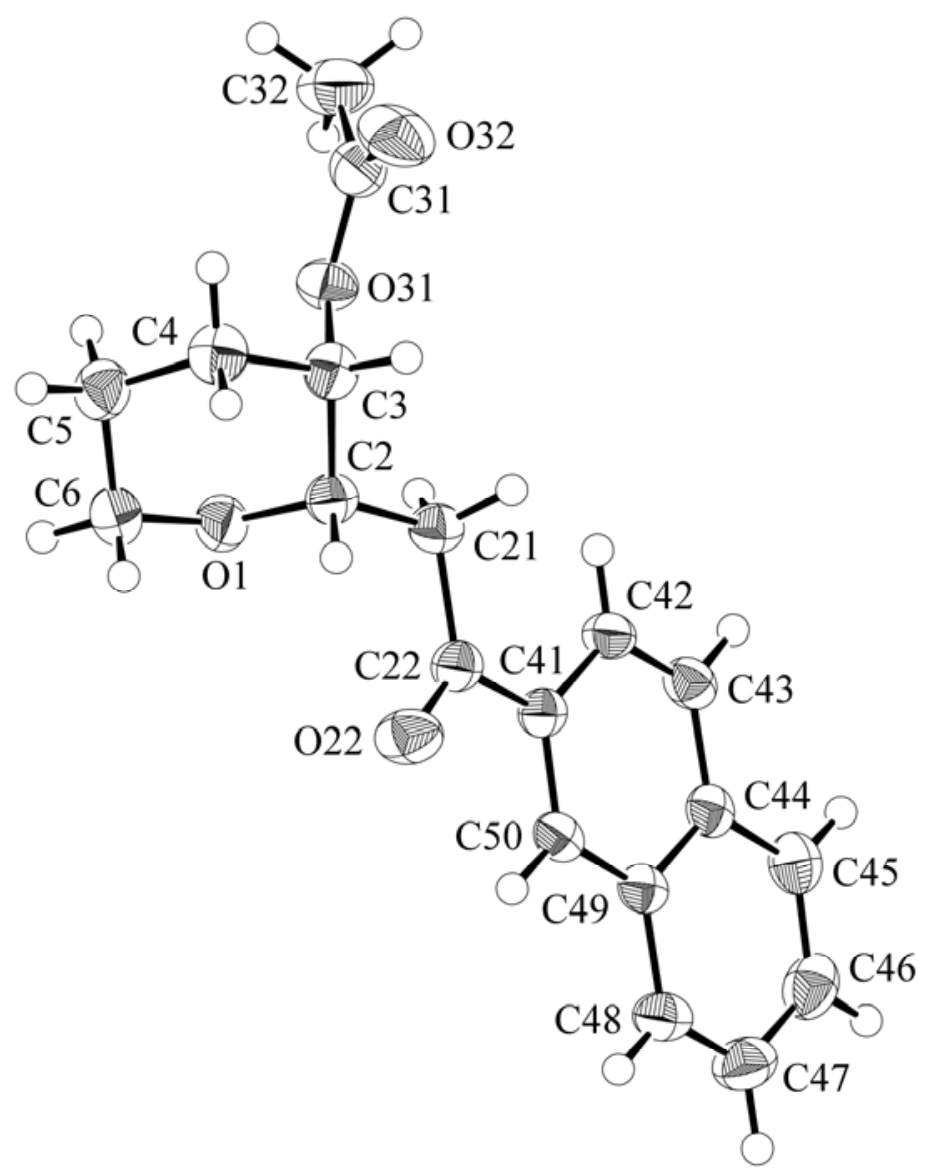

Molecular structure of $\mathbf{1 6 d},(S, S)$-enantiomer, showing the crystallographic numbering system employed. 


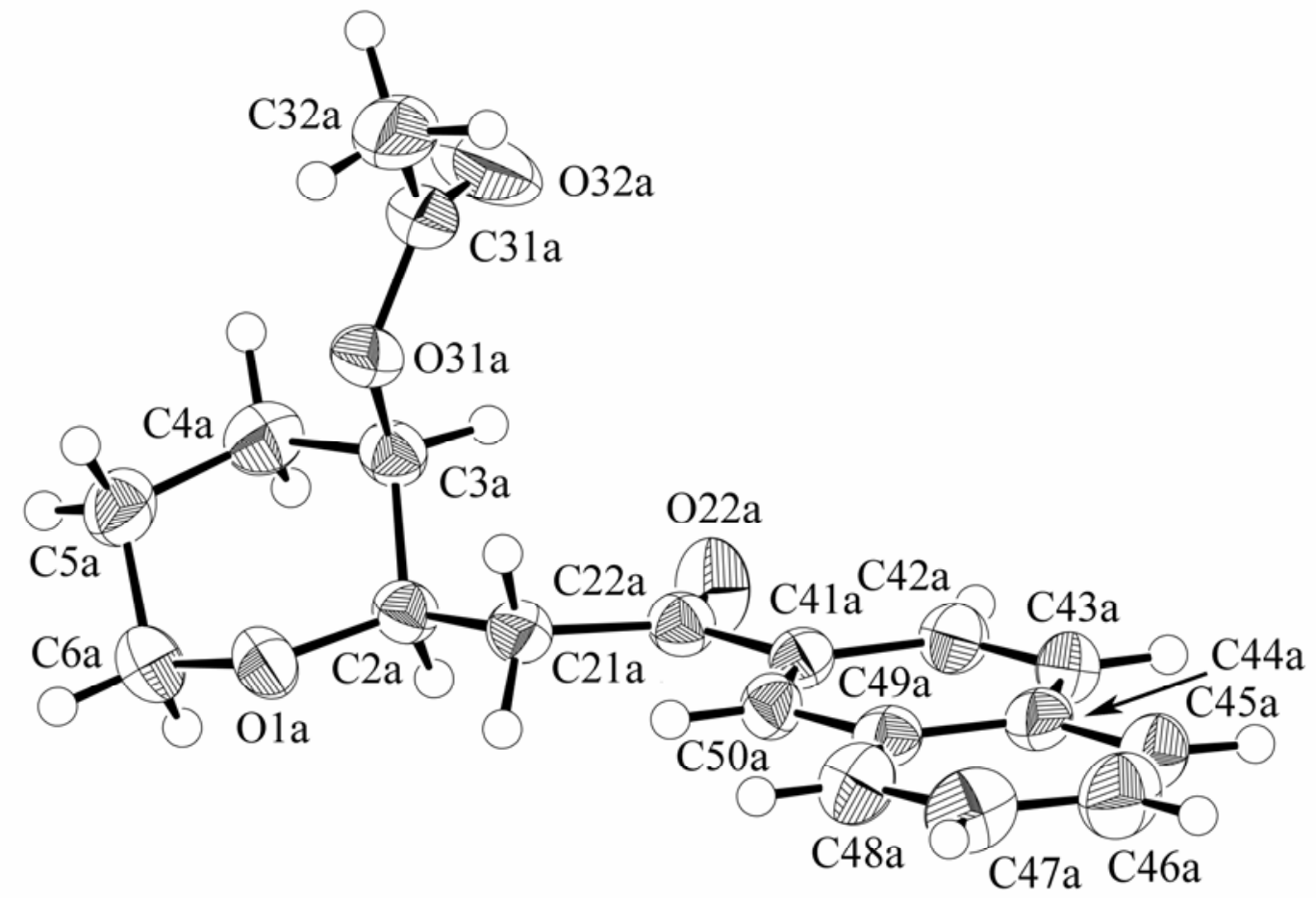

Molecular structure of $\mathbf{1 6 d},(R, R)$-enantiomer, showing the crystallographic numbering system employed.

Crystallographic data for 16d: $M=312.35, T=223(2) \mathrm{K}$, monoclinic, $P 2_{1} / c, a=$ 35.331(3), $b=10.4015(8), c=8.7744(7) \AA, \beta=96.181(2)^{\circ}, V=3205.8(4) \AA^{3}, Z=8$, $D_{\mathrm{x}}=1.294, F(000)=1328, \mu=0.090 \mathrm{~mm}^{-1}$, no. of unique data (Bruker AXS SMART CCD using Mo $\mathrm{K} \alpha$ radiation so that $\left.\theta_{\max }=30.1^{\circ}\right)=9346$, no. of parameters $=417, R$ $($ all data $)=0.154, w R($ all data $)=0.179, \rho=0.33$ e $\AA^{-3}$. The structure was solved by direct-methods (SIR92) and refined (anisotropic displacement parameters, $\mathrm{H}$ atoms in the riding model approximation and a weighting scheme $w=1 /\left[\sigma^{2}\left(F_{\mathrm{o}}{ }^{2}\right)+0.0717 P^{2}+\right.$ $0.4033 P]$ where $\left.P=\left(F_{\mathrm{o}}^{2}+2 F_{\mathrm{c}}^{2}\right) / 3\right)$ with SHELXL-97 on $F^{2}$ using all reflections. 
General procedure for the synthesis of 1,4-diketones. Refer to main paper.

7-Hydroxy-1-phenyl-1,4-heptanedione (14a) Colourless oil. $\mathrm{R}_{f} 0.42$ (3:2 ethyl acetate/hexanes). IR (neat) $3420,1712,1683,1597,1581,1448,1213,1024 \mathrm{~cm}^{-1} ;{ }^{1} \mathrm{H}$ $\operatorname{NMR}(300 \mathrm{MHz}) \delta 1.87$ (quintet, $J=6.6 \mathrm{~Hz}, 2 \mathrm{H}), 2.70(\mathrm{t}, J=6.6 \mathrm{~Hz}, 2 \mathrm{H}), 2.88(\mathrm{t}, J=$ $6.3 \mathrm{~Hz}, 2 \mathrm{H}), 3.31(\mathrm{t}, J=6.3 \mathrm{~Hz}, 2 \mathrm{H}), 3.66(\mathrm{t}, J=6.6 \mathrm{~Hz}, 2 \mathrm{H}), 4.92$ (br s, 1H), 7.427.49 (m, 2H), 7.53-7.60 (m, 1H), 7.95-8.00 (m, 2H). ${ }^{13} \mathrm{C}$ NMR (75 MHz) $\delta 26.5,32.4$ $36.1,39.3,61.8,127.9,128.4,133.0,136.5,198.7,210.0$; The diketone was unstable and was therefore fully characterised as its corresponding acetate $\mathbf{1 7 a}$.

4,7-Dioxo-7-phenylheptyl acetate (17a) Colourless oil. $\mathrm{R}_{f} \quad 0.25$ (3:7 ethyl acetate:hexanes). IR (neat) 1738, 1715, 1684, 1597, 1582, 1449, 1242, $1037 \mathrm{~cm}^{-1} ;{ }^{1} \mathrm{H}$ NMR (300 MHz) $\delta 1.95$ (quintet, $J=7.0 \mathrm{~Hz}, 2 \mathrm{H}), 2.05$ (s, 3H), 2.64 (t, $J=7.0 \mathrm{~Hz}$, 2H), $2.86(\mathrm{t}, J=6.3 \mathrm{~Hz}, 2 \mathrm{H}), 3.30(\mathrm{t}, J=6.3 \mathrm{~Hz}, 2 \mathrm{H}), 4.09$ (t, $J=7.0 \mathrm{~Hz}, 2 \mathrm{H}), 7.43-$ $7.49(\mathrm{~m}, 2 \mathrm{H}), 7.53-7.59(\mathrm{~m}, 1 \mathrm{H}), 7.96-8.00(\mathrm{~m}, 2 \mathrm{H}) ;{ }^{13} \mathrm{C} \mathrm{NMR}(75 \mathrm{MHz}) \delta 20.8,22.7$, 32.3, 36.1, 39.0, 63.5, 127.9, 128.5, 133.0, 136.6, 170.9, 198.4, 208.2; EIMS m/z: 262 (M+, 18), 234 (1), 219 (3), 202 (5), 176 (27), 161 (49), 133 (15), 105 (100), 77 (29), 43 (49); Anal. Calcd for $\mathrm{C}_{15} \mathrm{H}_{18} \mathrm{O}_{4}$ : C, 68.69; H, 6.92; Found C, 68.25; H, 6.89.

7-(4-Methoxyphenyl)-4,7-dioxoheptyl acetate (17b) Colourless solid; m.p. 47$49^{\circ} \mathrm{C} ; \mathrm{R}_{f} 0.20$ (1:3 ethyl acetate/hexanes); IR (neat) $17321715,1704,1674,1599 \mathrm{~cm}^{-}$ 1; ${ }^{1} \mathrm{H}$ NMR (300 MHz) $\delta 1.96$ (quintet, $\left.J=7.2 \mathrm{~Hz}, 2 \mathrm{H}\right), 2.03(\mathrm{~s}, 3 \mathrm{H}), 2.64$ (t, $J=7.2$ $\mathrm{Hz}, 2 \mathrm{H}), 2.84(\mathrm{t}, J=6.6 \mathrm{~Hz}, 2 \mathrm{H}), 3.26(\mathrm{t}, J=6.6 \mathrm{~Hz}, 2 \mathrm{H}), 3.87(\mathrm{~s}, 3 \mathrm{H}), 4.09(\mathrm{t}, J=7.2$ $\mathrm{Hz}, 2 \mathrm{H}), 6.92-6.95(\mathrm{~m}, 2 \mathrm{H}), 7.94-7.98(\mathrm{~m}, 2 \mathrm{H}) ;{ }^{13} \mathrm{C} \mathrm{NMR}(75 \mathrm{MHz}) \delta 20.9,22.8$, $32.1,36.3,39.2,55.4,63.6,113.7,129.8,130.3,163.6,171.0,197.0,208.5$; EIMS 
m/z: $292\left(\mathrm{M}^{+}, 6\right), 206$ (5), 191 (22), 163 (10), 135 (100), 97 (9), 71 (12), 57 (22), 43 (50); HRMS (EI) calcd for $\mathrm{C}_{16} \mathrm{H}_{20} \mathrm{O}_{5} 292.1311$, found 292.1317 .

7-(4-Bromophenyl)-4,7-dioxoheptyl acetate (17c) Colourless solid; m.p. 73-75 ${ }^{\circ} \mathrm{C} ; \mathrm{R}_{\mathrm{f}} 0.20$ (1:3 ethyl acetate/hexanes); IR (neat) 1728, 1710, 1680, 1583, 1568, 1276, $1037 \mathrm{~cm}^{-1} ;{ }^{1} \mathrm{H}$ NMR (300 MHz) $\delta 1.95$ (quintet, J = 7.2 Hz, 2H), $2.05(\mathrm{~s}, 3 \mathrm{H}), 2.64(\mathrm{t}$, $J=7.2 \mathrm{~Hz}, 2 \mathrm{H}), 2.86(\mathrm{t}, J=6.6 \mathrm{~Hz}, 2 \mathrm{H}), 3.24(\mathrm{t}, J=6.6 \mathrm{~Hz}, 2 \mathrm{H}), 4.09(\mathrm{t}, J=7.2 \mathrm{~Hz}$, 2H), $7.61(\mathrm{dd}, J=6.9,2.1 \mathrm{~Hz}, 2 \mathrm{H}), 7.83(\mathrm{dd}, J=6.9,2.1 \mathrm{~Hz}, 2 \mathrm{H}) ;{ }^{13} \mathrm{C}$ NMR $(75$ $\mathrm{MHz}) \delta 20.8,22.7,32.3,36.1,39.0,63.5,128.3,129.5,131.8,135.3,171.0,197.4$, 208.0; EIMS m/z: $342\left([\mathrm{M}+\mathrm{H}]^{+}, 5\right), 282$ (14), 254 (48), 239 (70), 211 (13), 183 (98), 157 (18), 129 (13), 104 (10), 87 (87), 69 (18), 43 (100); Anal. Calcd for $\mathrm{C}_{15} \mathrm{H}_{17} \mathrm{O}_{4} \mathrm{Br}$ : C, 52.80; H, 5.02: Found: C, 52.89; H, 5.02.

7-(2-Naphthyl)-4,7-dioxoheptyl acetate (17d) Colouless solid; Recrystallised from $\mathrm{CH}_{2} \mathrm{Cl}_{2}$ /heptane; m.p. $98-100^{\circ} \mathrm{C} ; \mathrm{R}_{f} 0.24$ (1:3 ethyl acetate/hexanes). IR (nujol) 1731, 1712, 1681, $1624 \mathrm{~cm}^{-1} ;{ }^{1} \mathrm{H} \mathrm{NMR}(300 \mathrm{MHz}) \delta 1.97$ (quintet, $\left.J=7.2 \mathrm{~Hz}, 2 \mathrm{H}\right), 2.06$ (s, 3H), $2.67(\mathrm{t}, J=7.2 \mathrm{~Hz}, 2 \mathrm{H}), 2.92(\mathrm{t}, J=6.3 \mathrm{~Hz}, 2 \mathrm{H}), 3.45(\mathrm{t}, J=6.3 \mathrm{~Hz}, 2 \mathrm{H}), 4.11(\mathrm{t}$, $J=7.2 \mathrm{~Hz}, 2 \mathrm{H}), 7.53-7.63(\mathrm{~m}, 2 \mathrm{H}), 7.86-7.91(\mathrm{~m}, 2 \mathrm{H}), 7.95-7.98(\mathrm{~m}, 1 \mathrm{H}), 8.03(\mathrm{dd}, J$ $=8.7,1.8 \mathrm{~Hz}, 1 \mathrm{H}), 8.52(\mathrm{br} \mathrm{s}, 1 \mathrm{H}) ;{ }^{13} \mathrm{C} \mathrm{NMR}(75 \mathrm{MHz}) \delta 20.8,22.8,32.4,36.3,39.1$, $63.6,123.7,126.7,127.7,128.3,128.4,129.5,129.7,132.5,133.9,135.6,170.9$, 198.3, 208.3; EIMS m/z: $312\left(\mathrm{M}^{+}, 13\right), 211$ (19), 155 (100), 127 (33) 43 (28). Anal. Calcd for $\mathrm{C}_{19} \mathrm{H}_{20} \mathrm{O}_{4}$ : C, 73.06; H, 6.45; Found C, 73.01; H, 6.36. 


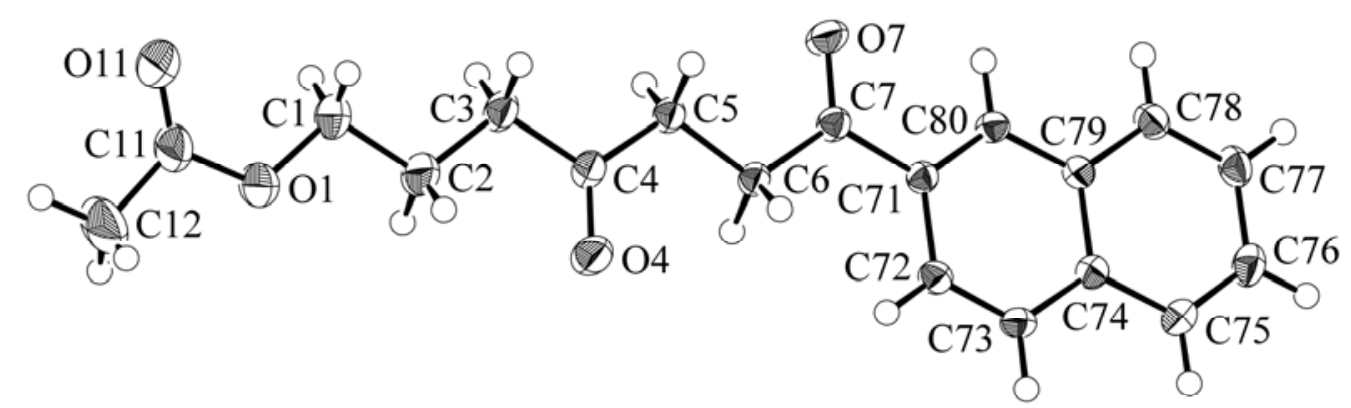

Molecular structure of $\mathbf{1 7 d}$ showing the crystallographic numbering system employed.

Crystallographic data for $17 \mathbf{d}: M=312.35, T=223(2) \mathrm{K}$, monoclinic, $P 2_{1} / c, a=$ 5.5546(5), $b=39.599(3), c=7.3808(6) \AA, \beta=98.301(2)^{\mathrm{o}}, V=1606.4(2) \AA^{3}, Z=4$, $D_{\mathrm{x}}=1.291, F(000)=664, \mu=0.090 \mathrm{~mm}^{-1}$, no. of unique data (Bruker AXS SMART CCD using Mo K $\alpha$ radiation so that $\left.\theta_{\max }=30.1^{\circ}\right)=4688$, no. of parameters $=210, R$ $($ all data $)=0.096, w R($ all data $)=0.189, \rho=0.51$ e $\AA^{-3}$. The structure was solved by direct-methods (SIR92) and refined (anisotropic displacement parameters, $\mathrm{H}$ atoms in the riding model approximation and a weighting scheme $w=1 /\left[\sigma^{2}\left(F_{\mathrm{o}}{ }^{2}\right)+0.1068 P^{2}+\right.$ 0.097P] where $\left.P=\left(F_{\mathrm{o}}{ }^{2}+2 F_{\mathrm{c}}{ }^{2}\right) / 3\right)$ with SHELXL-97 on $F^{2}$ using all reflections.

1-Methyl-4,7-dioxo-7-phenylheptyl acetate (23). To a solution of 1,2-dioxine 10g (307 $\mathrm{mg}, 1.31 \mathrm{mmol})$ in dichloromethane $(10 \mathrm{~mL})$ was added triethylamine (20 drops) and the solution stirred over night. The volatiles were removed in vacuo and pyridine (1.6 mL, $19.60 \mathrm{mmol}$ ), acetic anhydride (1.1 mL, $11.80 \mathrm{mmol}$ ) and DMAP (32 mg, $0.26 \mathrm{mmol}$.) were added and the solution stirred overnight. The solution was then taken up in dichloromethane $(20 \mathrm{~mL})$ and extracted with water $(30 \mathrm{~mL})$, dried $\left(\mathrm{MgSO}_{4}\right)$, filtered and the volatiles removed in vacuo. The crude diketone was 
purified by column chromatography to yield $322 \mathrm{mg}, 89 \%$ of 1,4-diketone 23 as a colourless oil; $\mathrm{R}_{f}=0.35$ (1:3 ethylacetate/hexane); IR (neat) 1731, 1714, 1682, 1598, 1580, $12451019 \mathrm{~cm}^{-1} ;{ }^{1} \mathrm{H}$ NMR (300 MHz) $\delta 1.23(\mathrm{~d}, J=6.3 \mathrm{~Hz}, 1 \mathrm{H}), 1.82-1.92(\mathrm{~m}$, 2H), $2.04(\mathrm{~s}, 3 \mathrm{H}), 2.51-2.68(\mathrm{~m}, 2 \mathrm{H}), 2.77-2.94(\mathrm{~m}, 2 \mathrm{H}), 3.21-3.39(\mathrm{~m}, 2 \mathrm{H}), 4.92$ (ddq, $J=6.3,6.3,6.3 \mathrm{~Hz}, 1 \mathrm{H}), 7.43-7.49(\mathrm{~m}, 2 \mathrm{H}), 7.54-7.59(\mathrm{~m}, 1 \mathrm{H}), 7.96-7.80(\mathrm{~m}$, $2 \mathrm{H}) ;{ }^{13} \mathrm{C}$ NMR $(75 \mathrm{MHz}) \delta 20.0,21.2,29.7,32.4,36.2,38.7,70.2,128.0,128.5$, 133.1, 136.7, 170.6, 198.5, 208.3; MS (EI) m/z (\%): $276\left(\mathrm{M}^{+}, 5\right), 176(28), 161$ (52) 105 (100), 77 (41), 43 (47); HRMS (EI) calcd for $\mathrm{C}_{16} \mathrm{H}_{20} \mathrm{O}_{4}$ requires 276.1362, found 276.1366 .

3-(5-Phenyl-2-furyl)-1-propanol (18a). ${ }^{13}$ Colourless oil; $\mathrm{R}_{f} \quad 0.53 \quad$ (1:1 ethyl acetate/hexanes); IR (neat) 3340, 2945, 2948, 1610, 1595, 1578, 1548, 1487, 1065, 1016, $759 \mathrm{~cm}^{-1} ;{ }^{1} \mathrm{H}$ NMR (300 MHz) $\delta 1.60($ br s, $1 \mathrm{H}), 1.91-2.04(\mathrm{~m}, 2 \mathrm{H}), 2.79(\mathrm{t}, J=$ $7.8 \mathrm{~Hz}, 2 \mathrm{H}), 3.73(\mathrm{t}, J=6.3 \mathrm{~Hz}, 2 \mathrm{H}), 6.09(\mathrm{dt}, J=3.3,0.9 \mathrm{~Hz}, 1 \mathrm{H}), 6.54(\mathrm{~d}, J=3.3$ $\mathrm{Hz}, 1 \mathrm{H}), 7.18-7.24(\mathrm{~m}, 1 \mathrm{H}), 7.32-7.38(\mathrm{~m}, 2 \mathrm{H}), 7.60-7.64(\mathrm{~m}, 2 \mathrm{H}) ;{ }^{13} \mathrm{C}$ NMR $(75$ MHz) $\delta 24.5,31.0,62.1,105.6,107.3,123.3,126.8,128.6,131.0,152.4,155.3$.

\section{General procedure for the synthesis of trans $\gamma$-hydroxy enones 11a-g. To a 0.1}

M solution of 1,2-dioxine 10a-g in acetone was added triphenylphosphine ( 0.5 equiv) followed by drop-wise addition of triethylamine (1 drop / $10 \mathrm{mg}$ of 1,2-dioxine). The solution was stirred for 2 hours, closely monitored by tlc, once complete the volatiles were removed in vacuo and the crude trans $\gamma$-hydroxy enone $\mathbf{1 1}$ was purified by column chromatography. The trans $\gamma$-hydroxy enones were utilized immediately due to instability. 
(E)-4,7-Dihydroxy-1-phenyl-2-hepten-1-one (11a) Yield: 134 g 84\%. Colourless oil. $\mathrm{R}_{f} 0.11$ (3:2 ethyl acetate/hexanes). Purified by flash chromatography (100\% ethyl acetate).

(E)-4,7-Dihydroxy-1-(4-methoxyphenyl)-2-hepten-1-one (11b) Yield: 299 mg, 84\%; Colourless oil; $\mathrm{R}_{f} 0.20$ (1:3 ethyl acetate/diethyl ether). Purified by flash chromatography (7:13 ethyl acetate/diethyl ether).

(E)-1-(4-Bromophenyl)-4,7-dihydroxy-2-hepten-1-one (11c) Yield: $302 \mathrm{mg}$, 58\%; Colourless oil; $\mathrm{R}_{f} 0.20$ (1:3 ethyl acetate/diethyl ether). Purified by flash chromatography.

(E)-4,7-Dihydroxy-1-(2-naphthyl)-2-hepten-1-one (11d) Yield: 375 mg, 83\%; $\mathrm{R}_{f}$ 0.40 (ethyl acetate). Purified by flash chromatography.

(E)-4,7-Dihydroxy-7-methyl-1-phenyl-2-octen-1-one (11e) Yield: 47.7 mg, 80\%; Colourless oil; $\mathrm{R}_{f} 0.47$ (ethyl acetate). Purified by flash chromatography.

(E)-4,7-dihydroxy-1,7,7-triphenyl-2-hepten-1-one (11f) Yield: 30 mg, 55\%; Pale yellow solid; $\mathrm{R}_{f} 0.35$ (2:3 ethyl acetate/hexanes). Purified by flash chromatography.

(E)-4,7-dihydroxy-1-phenyl-2-octen-1-one (11g). Yield: 793 mg, 85\%; Colourless oil; $\mathrm{R}_{f} 0.16$ (7:3 ethyl acetate/hexanes).

\section{References}


1. Charette, A. B.; Molinaro, C.; Brochu, C. J. Am. Chem. Soc., 2001, 123, 12168.

2. Huang, Z-Z.; Tang, Y. J. Org. Chem., 2002, 67, 5320.

3. Xu, C.; Chen, G.; Fu, C.; Huang, X. Synth. Commun., 1995, 25, 2229.

4. Hammond, M. L.; Zambias, R. A.; Chang, M. N.; Jensen, N. P.; McDonald, J.; Thompson, K.; Boulton, D. A.; Kopka, I. E.; Hand, K. M.; Opas, E. E.; Luell, S.; Bach, T.; Davies, P.; MacIntyre, D. E.; Bonney, R. J.; Humes, J. L. J. Med. Chem., 1990, 33, 908.

5. Corey, E. J.; Schmidt, G. Tetrahedron Lett. 1979, 5, 399.

6. Bellassoued, M.; Majidi, A. J. Org. Chem., 1993, 58, 2517.

7. Chuzel, O.; Piva, O. Synth. Commun., 2003, 33, 393.

8. Battistuzzi, G.; Cacchi, S.; Fabrizi, G. Org. Lett. 2003, 5, 777.

9. Merlic, C. A.; Aldrich, C. C.; Albanese-Walker, J.; Saghatelian, A.; Mammen, J. J. Org. Chem., 2001, 66, 129

10. Sodeoka, M.; Yamada, H.; Shibasaki, M. J. Am. Chem. Soc., 1990, 112, 4906.

11. Corey, E. J.; Venkateswarlu, A. J. Am. Chem. Soc., 1972, 94, 6190.

12. Matsumoto, M.; Dobasshi, S.; Kuroda, K.; Kondo, K. Tetrahedron 1985, 41, 2147.

13. Thewalt, K.; Rudolph, W. Ber. 1963, 96, 136. 


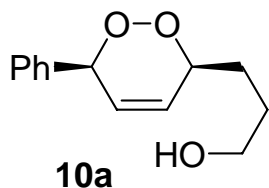

$892 \cdot 82=$
SS6
$62=$

oIs.29-

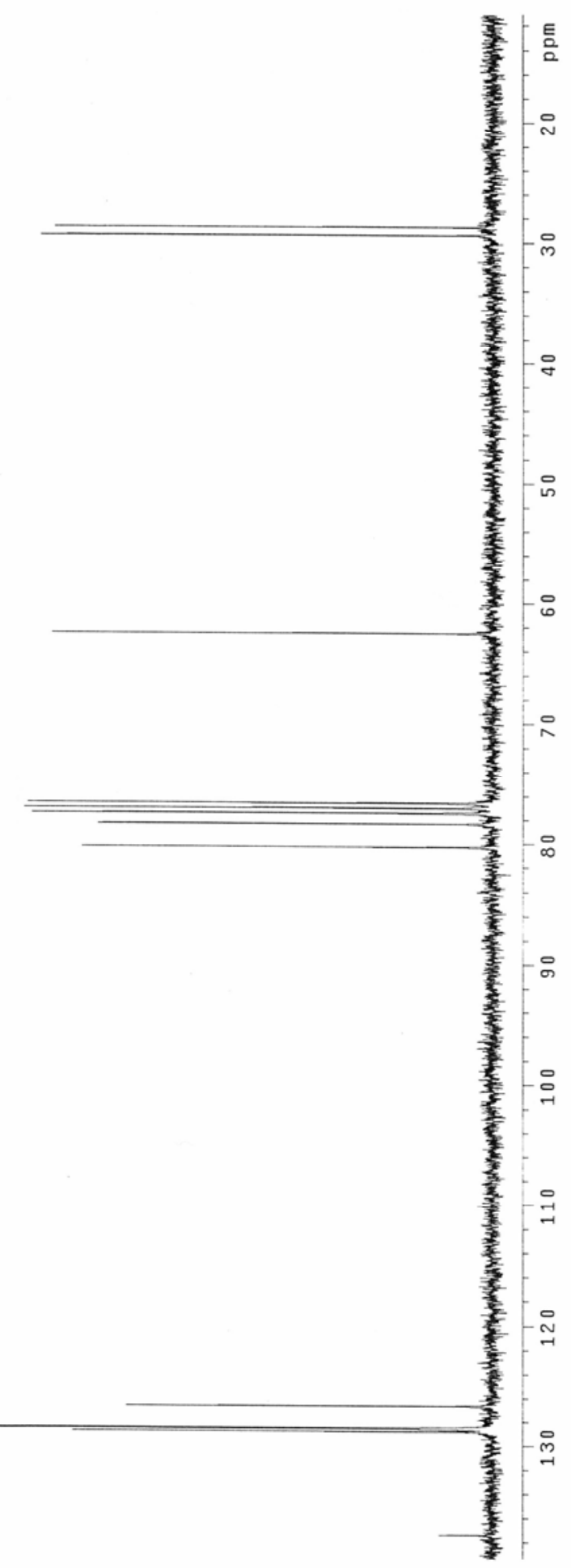

$\angle 09 \cdot 925-$

9ES. 821

ISS.82I-

$06 \varepsilon^{\circ} \angle 8 \mathrm{I}$ 
$p-\mathrm{MeOPh}-\underbrace{\mathrm{O}-\mathrm{O}}_{\mathbf{H O}}$

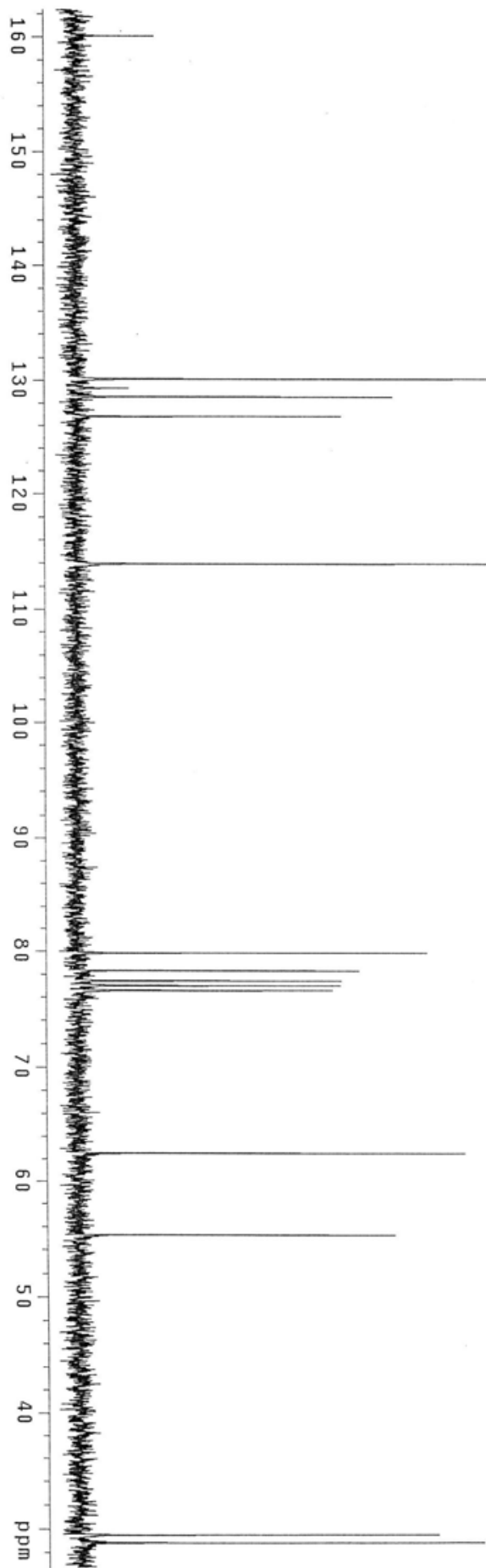

160.089

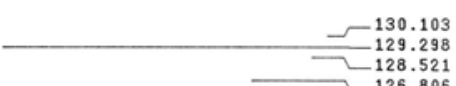

-128.521
-126.805

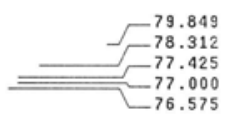

$-62.475$

55.249

$-29.504$ 

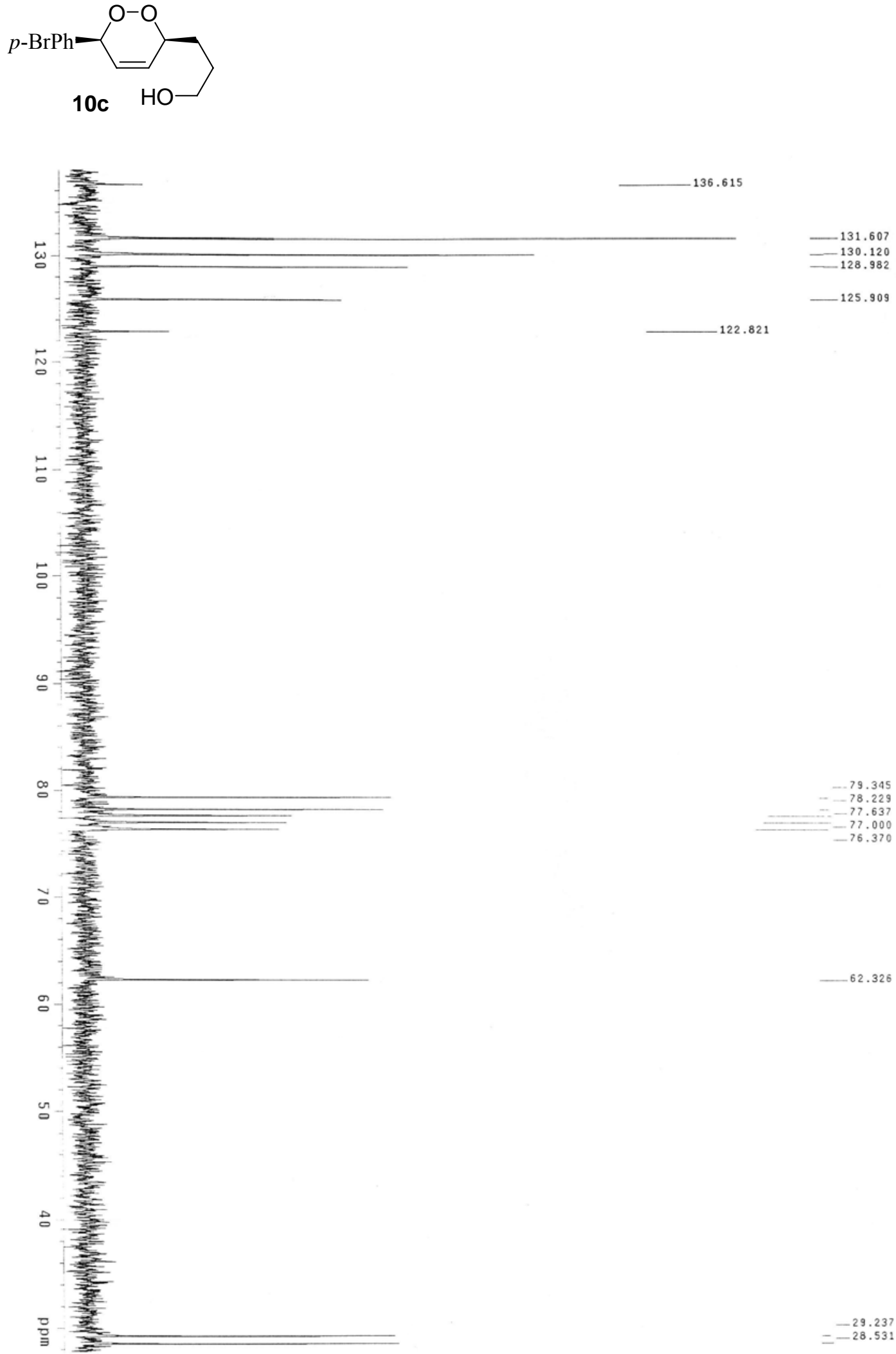
naphthyl

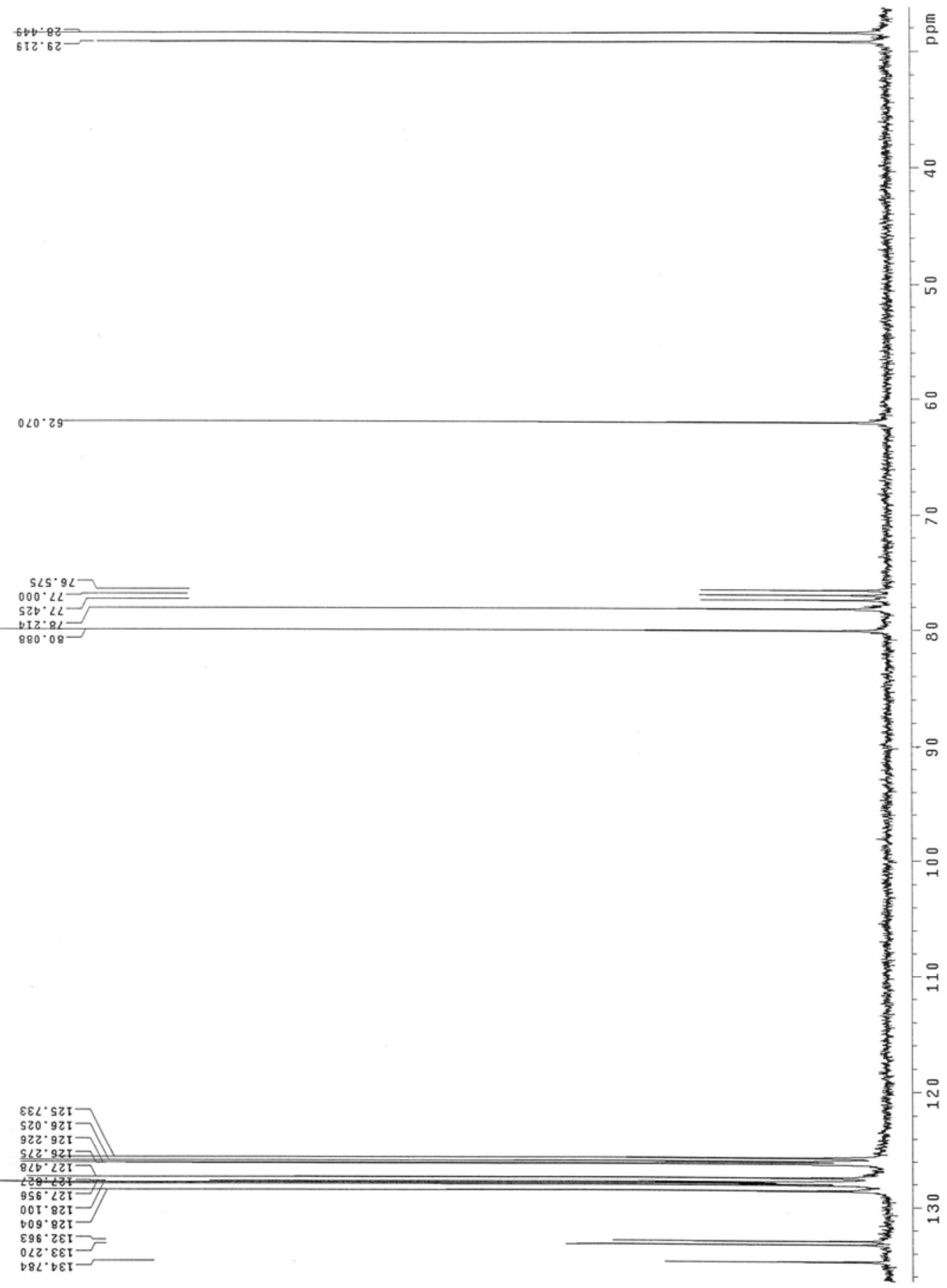



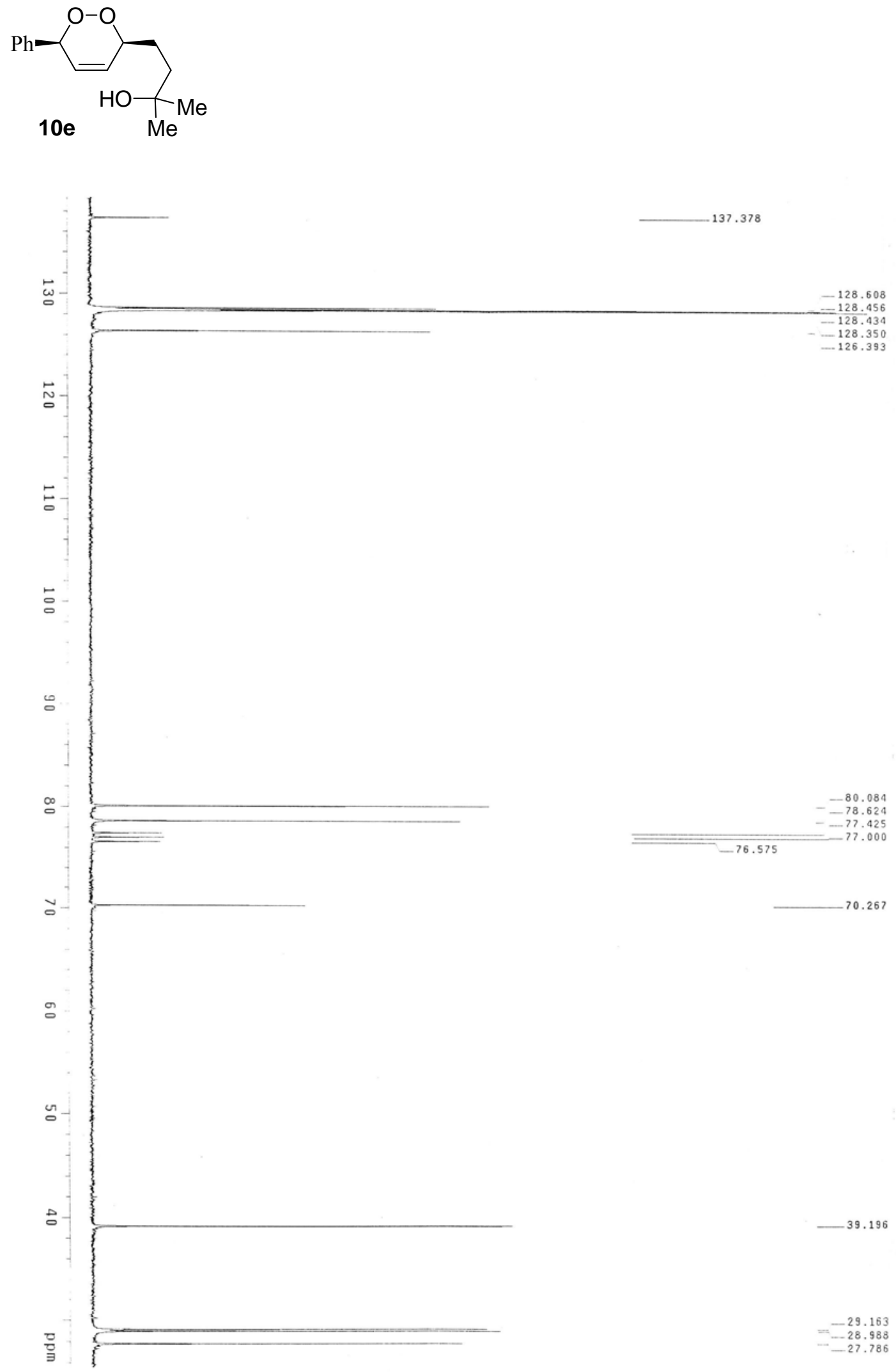

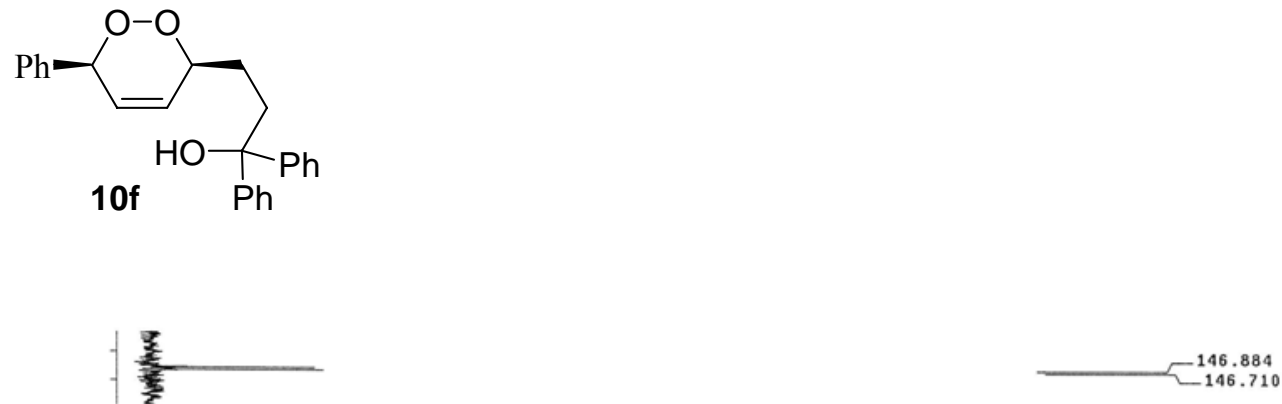

品-

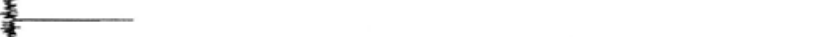

.

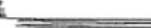

$F_{128.494}^{128.702}$

$-128.157$

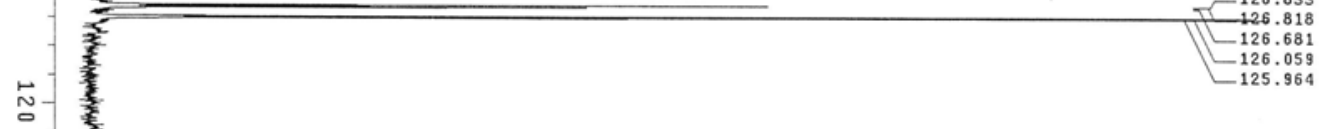

N

함

:

$\circ-$

$\infty$
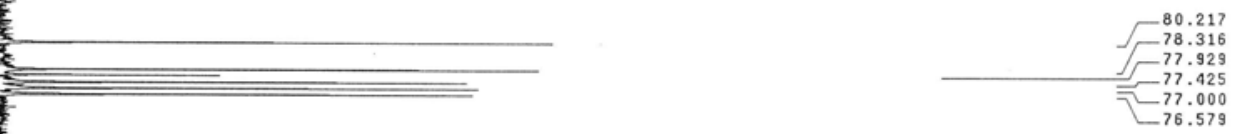

。.

ज

吕-

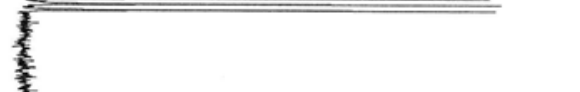

77.000
-76.579

$\therefore$

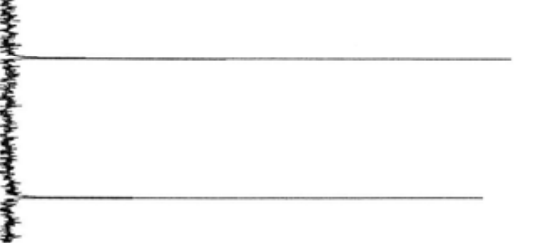

$-37.432$

$-27.75$ 

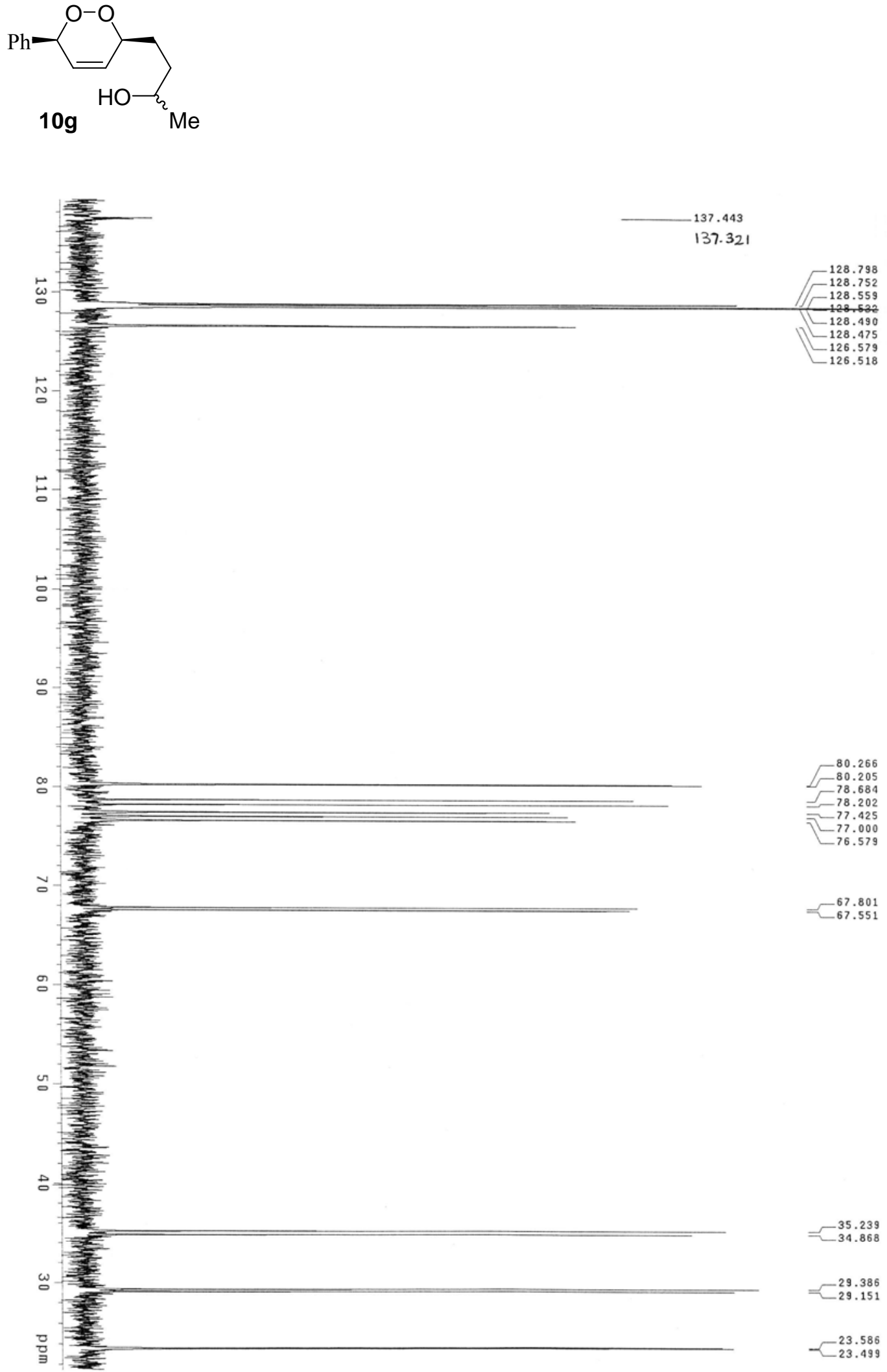

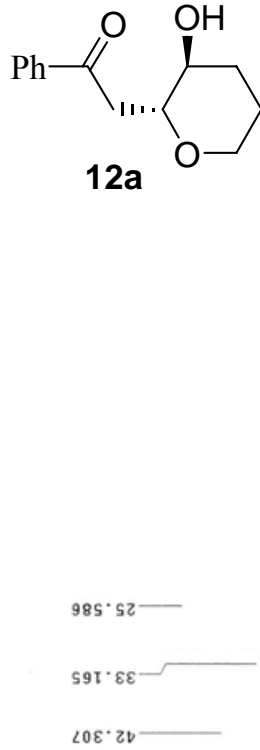

$818 . \angle 9$

$269^{\circ} 02-$
812.02

$68 L .9 L$

$000^{\circ}: 24$

616.82

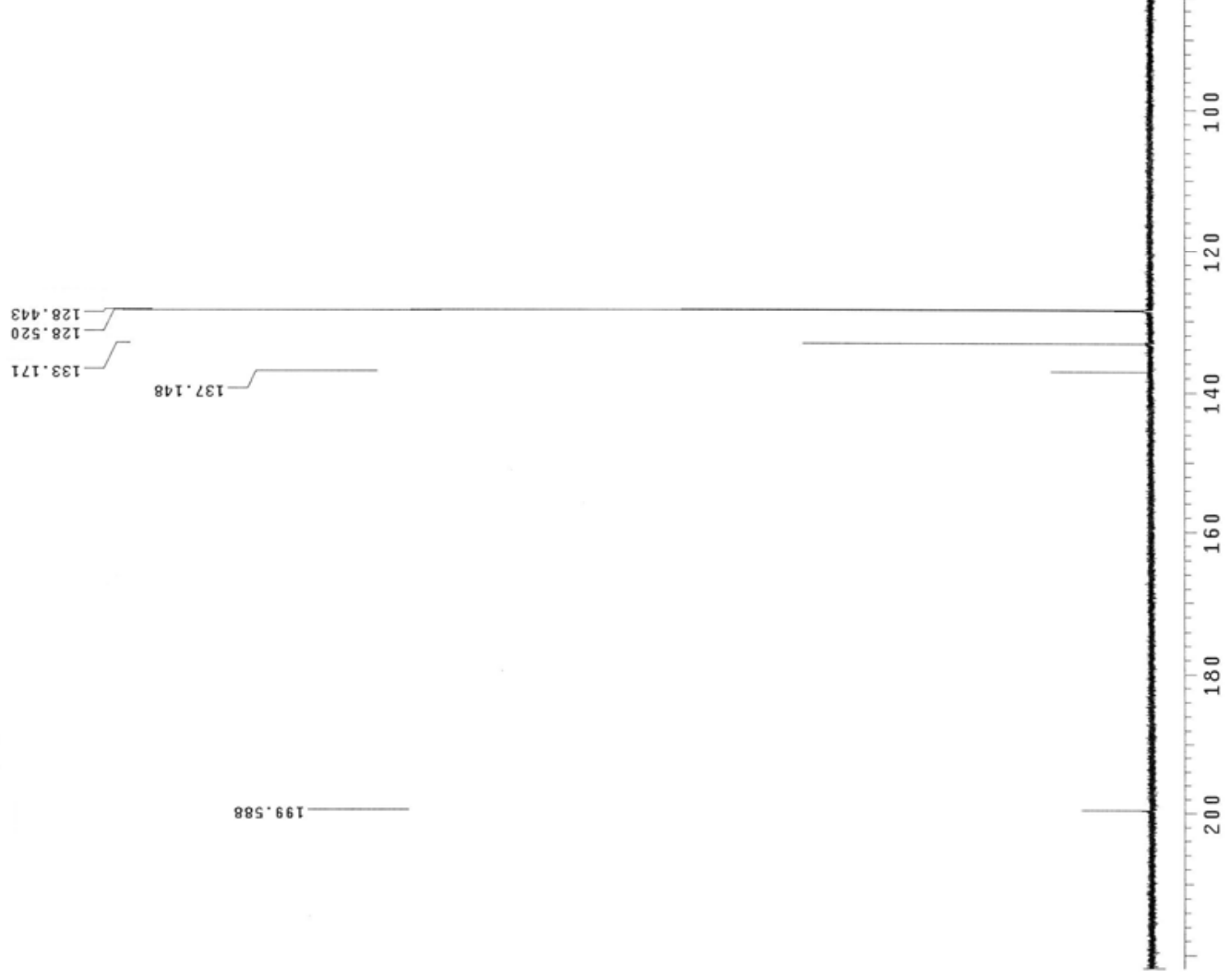


S34
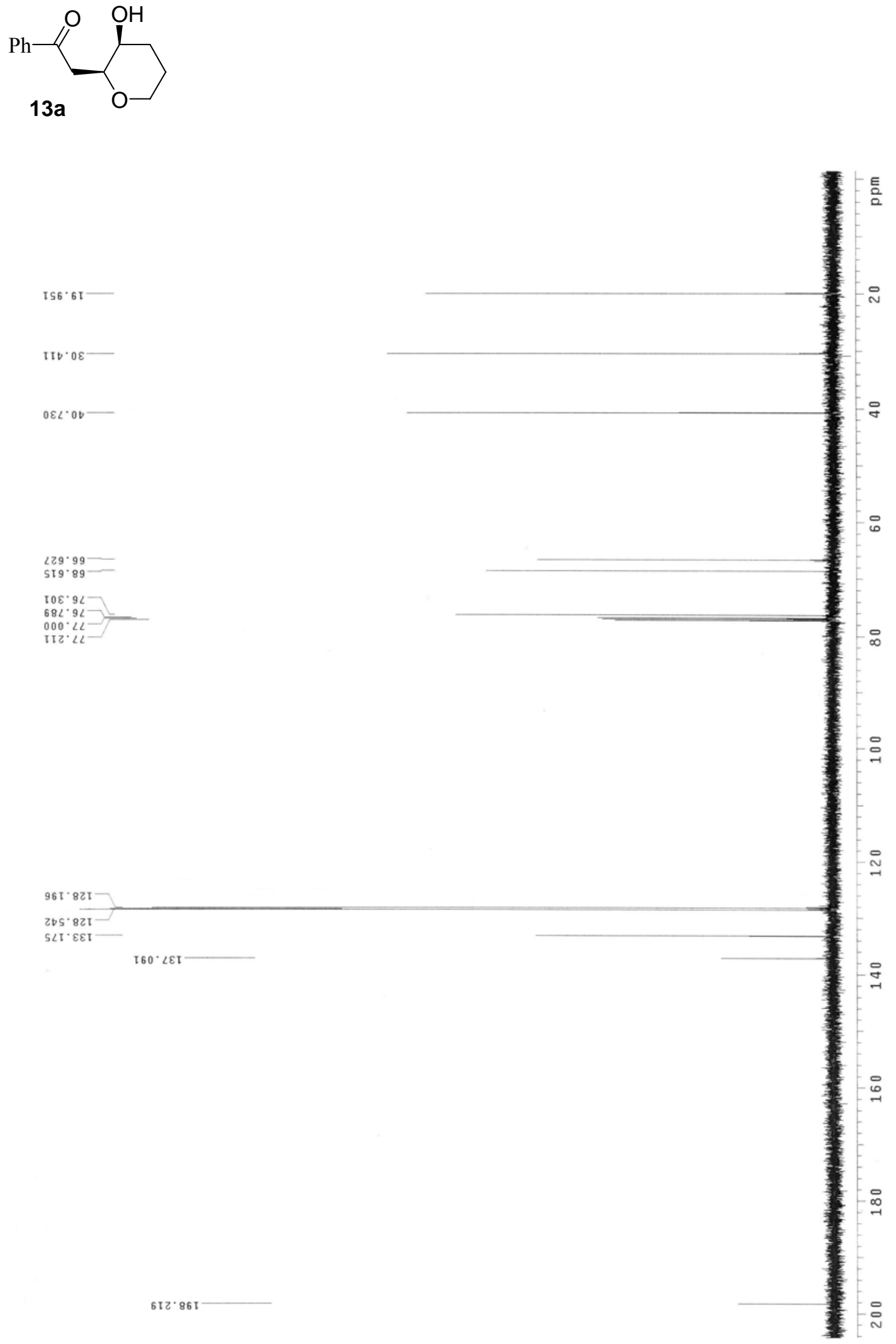

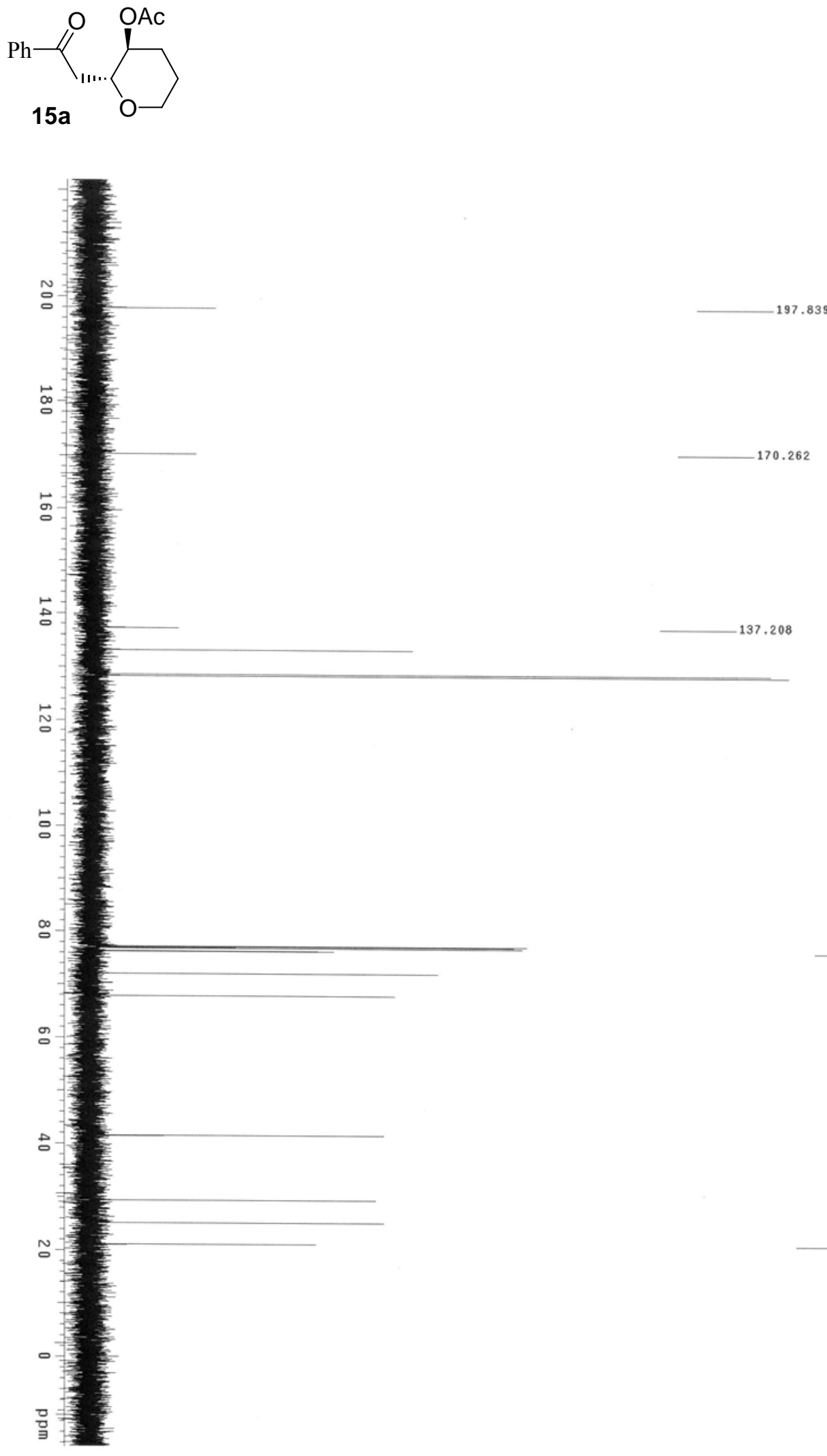

$-170.262$

$-137.208$ 133.121
$-\quad 128.561$
$-\quad 128.292$ 

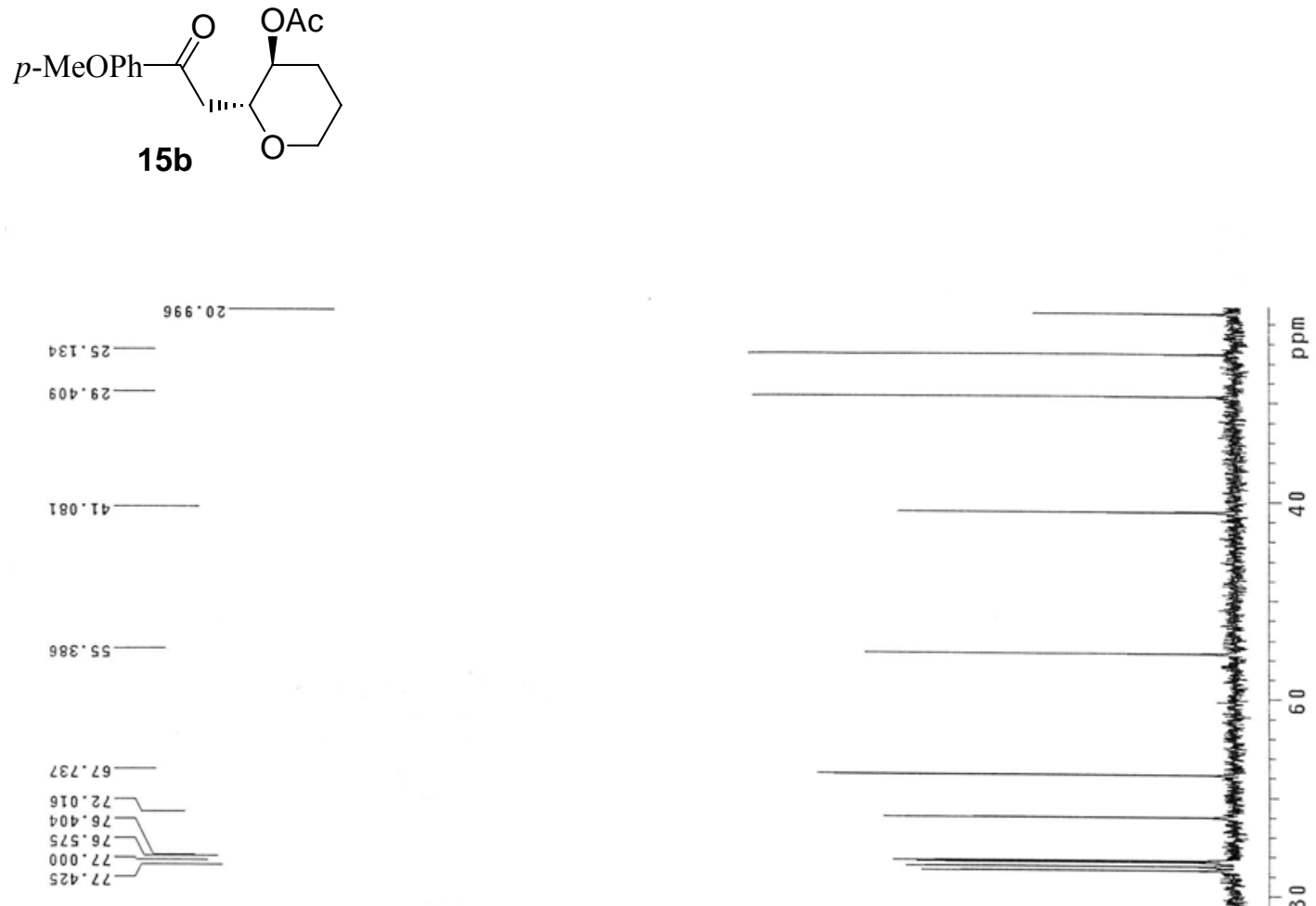

$299^{\circ} \varepsilon \mathrm{EIT}$

69S.08ז -
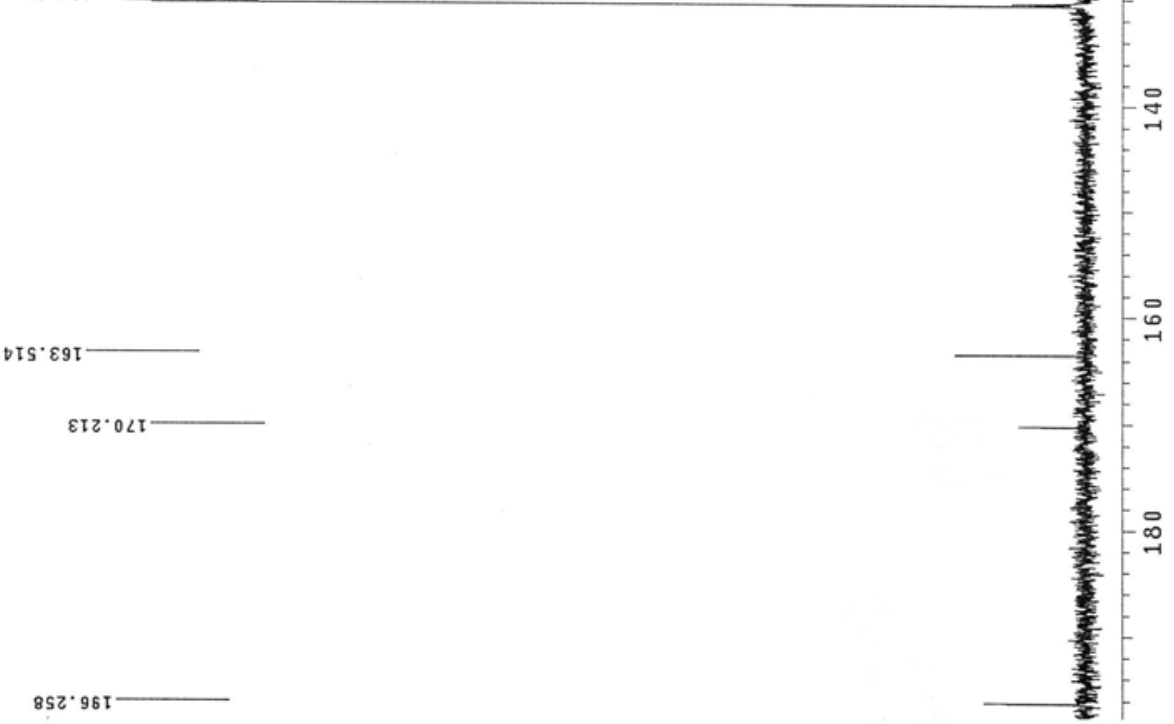
- $\mathrm{BrPh}-\mathrm{1}_{\mathrm{O}-\mathrm{C}}^{\mathrm{OAc}}$
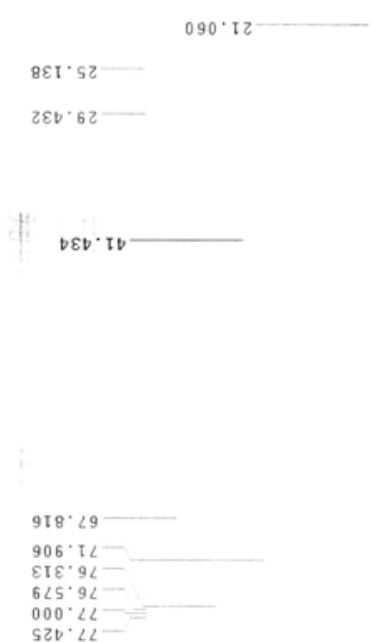

$9 \mathrm{S8} \cdot 62 \mathrm{I}^{-}$

โรย' $82 \tau$

$0 Z 0 \cdot 9 \varepsilon \tau$

$D 6 \mathrm{I} \cdot 0<\mathrm{I}$

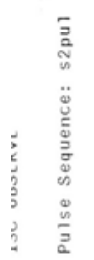

$806.96 T^{-}$

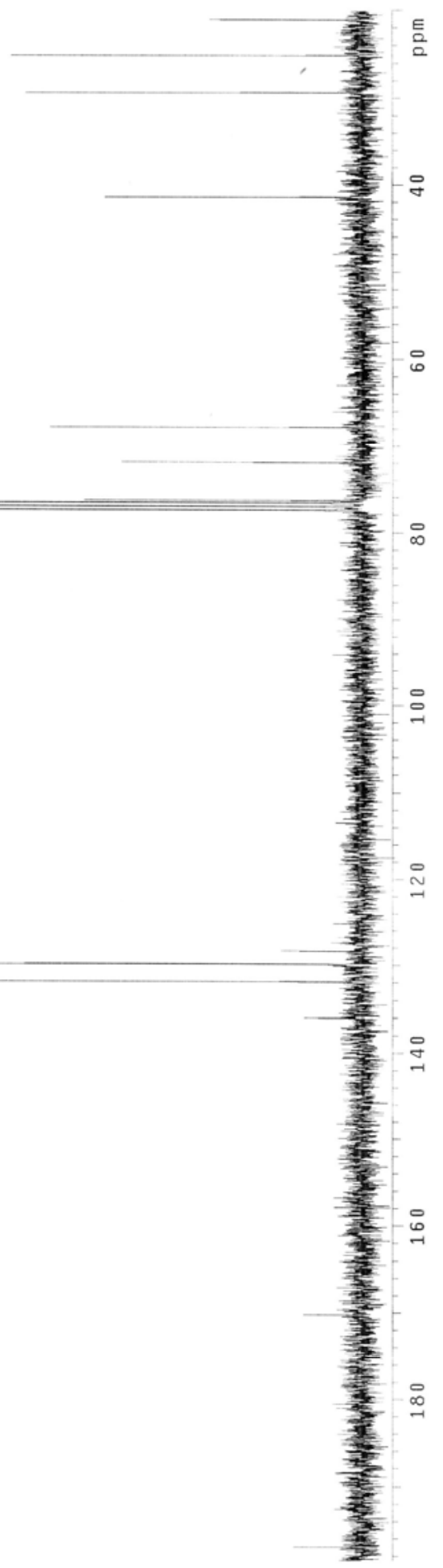


naphthyl

$696.02-$
260.52

$88 \varepsilon^{\circ} 62$

$D E D^{\circ}$ Tt

$\stackrel{\circ}{\circ}$

8

OT $\angle 2 \angle 9$

$656^{\circ} \mathrm{TL}$

I $5 \varepsilon^{\circ} \cdot 9 L$

$000^{\circ} .2-$

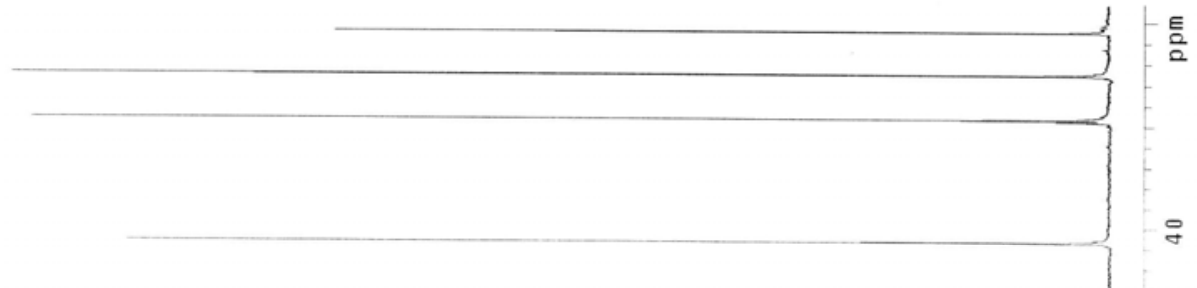

틈

\section{(5)}

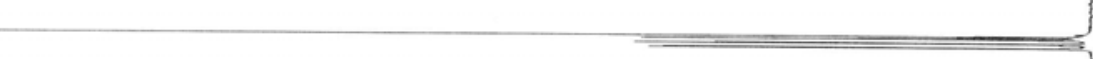

$998^{\circ} \varepsilon z \mathrm{I}$

\begin{tabular}{l}
$689 \cdot 9 Z \mathrm{I}$ \\
$9 Z 9 \cdot \angle Z \mathrm{I}$ \\
\hline $08 \cdot 8 \mathrm{~T}$
\end{tabular}

น08. $85 \mathrm{~T}$

b8E. $82 \mathrm{II}$

$200^{\circ} 08 \mathrm{I}-$

8Z0. $28 \mathrm{I}$

SDS.
ZISI
ISI-

$89 T^{\circ} 0 \angle \mathrm{T}$

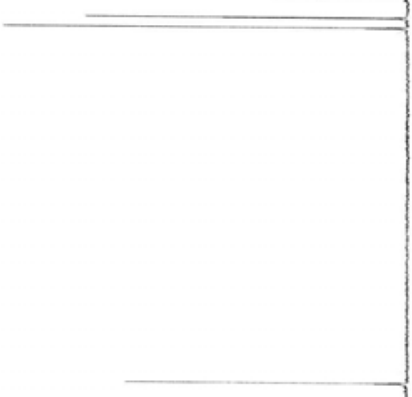



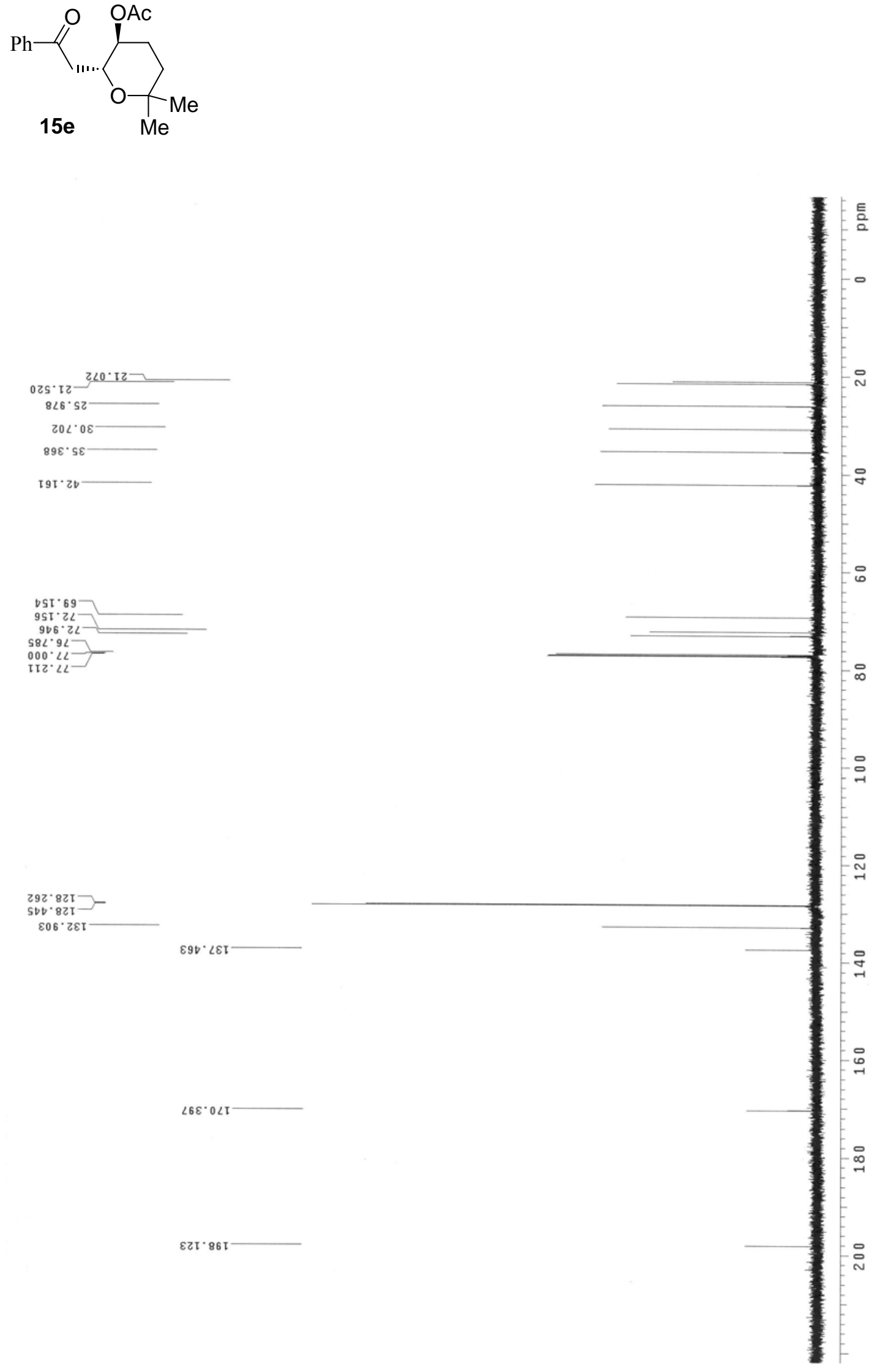

DSI. $69-$
$9 S I \cdot 2 L-$
$906.2 L-$
$S 8 L .92-$

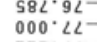

เป2. 22

$292^{\circ} 821-$
SDD. $821-$

$806^{\circ} 2 \varepsilon \mathrm{I}-$

$\varepsilon 9 b \cdot \angle \varepsilon \downarrow$

$\angle 6 \varepsilon^{\circ} 0 \angle I^{-}$

$\varepsilon 2 \tau \cdot 86 \mathrm{I}$ 

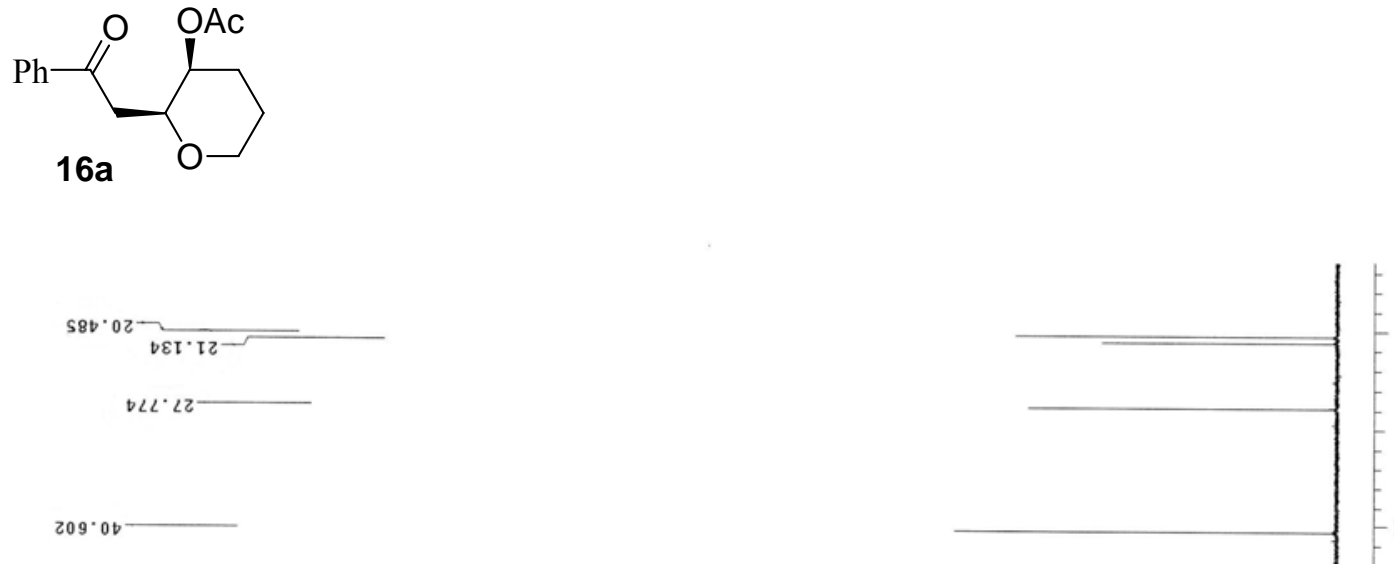

$209 \cdot 0 b$

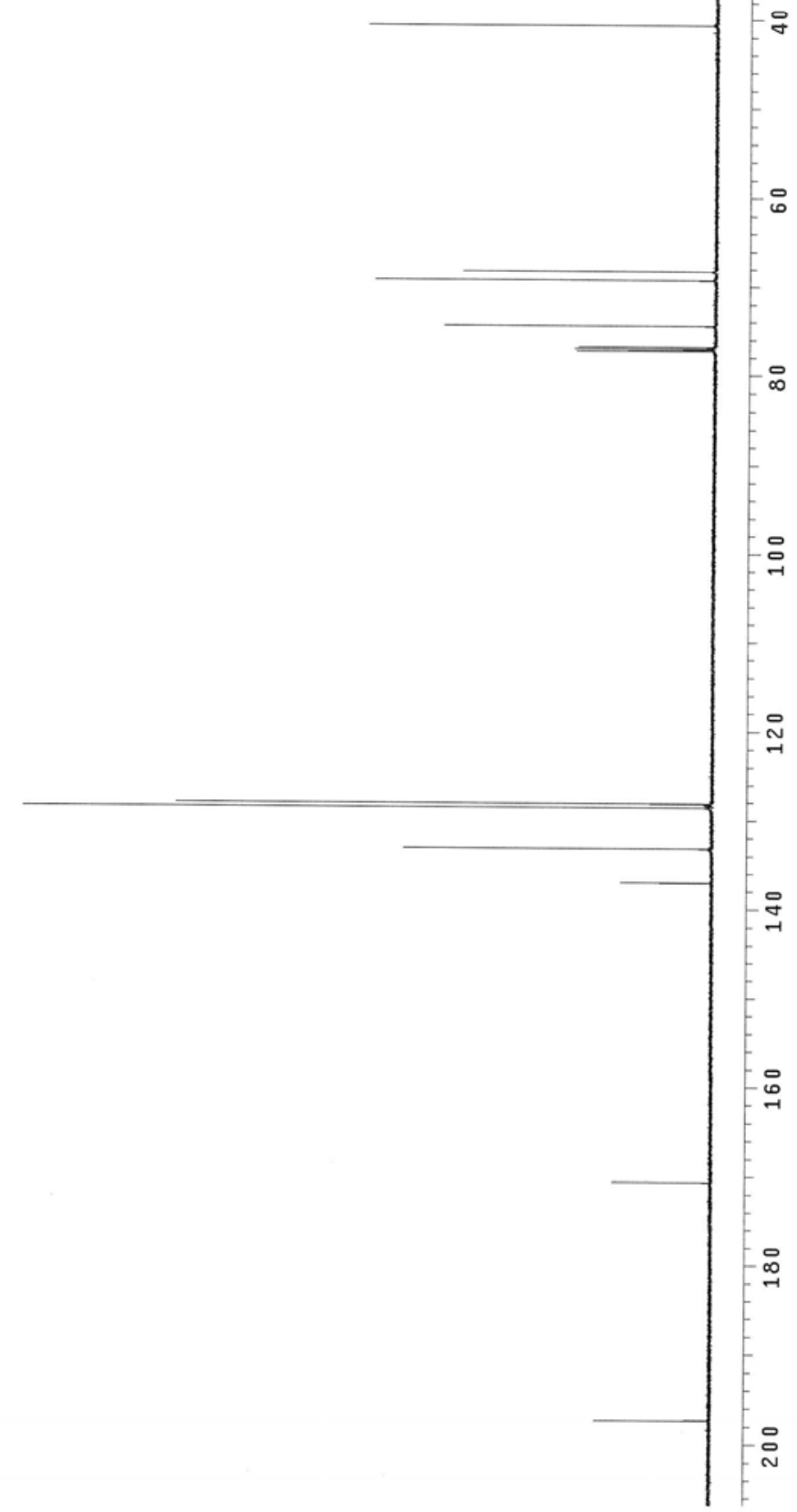

$201 \cdot 821 \square$
$825 \cdot 821-$

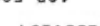

$\forall S I \cdot \varepsilon \varepsilon I$

$286^{\circ} 9 \varepsilon \mathrm{I}$

$6 \angle S^{\circ} 0 \angle I$

$262 \cdot 26 \mathrm{I}$ 

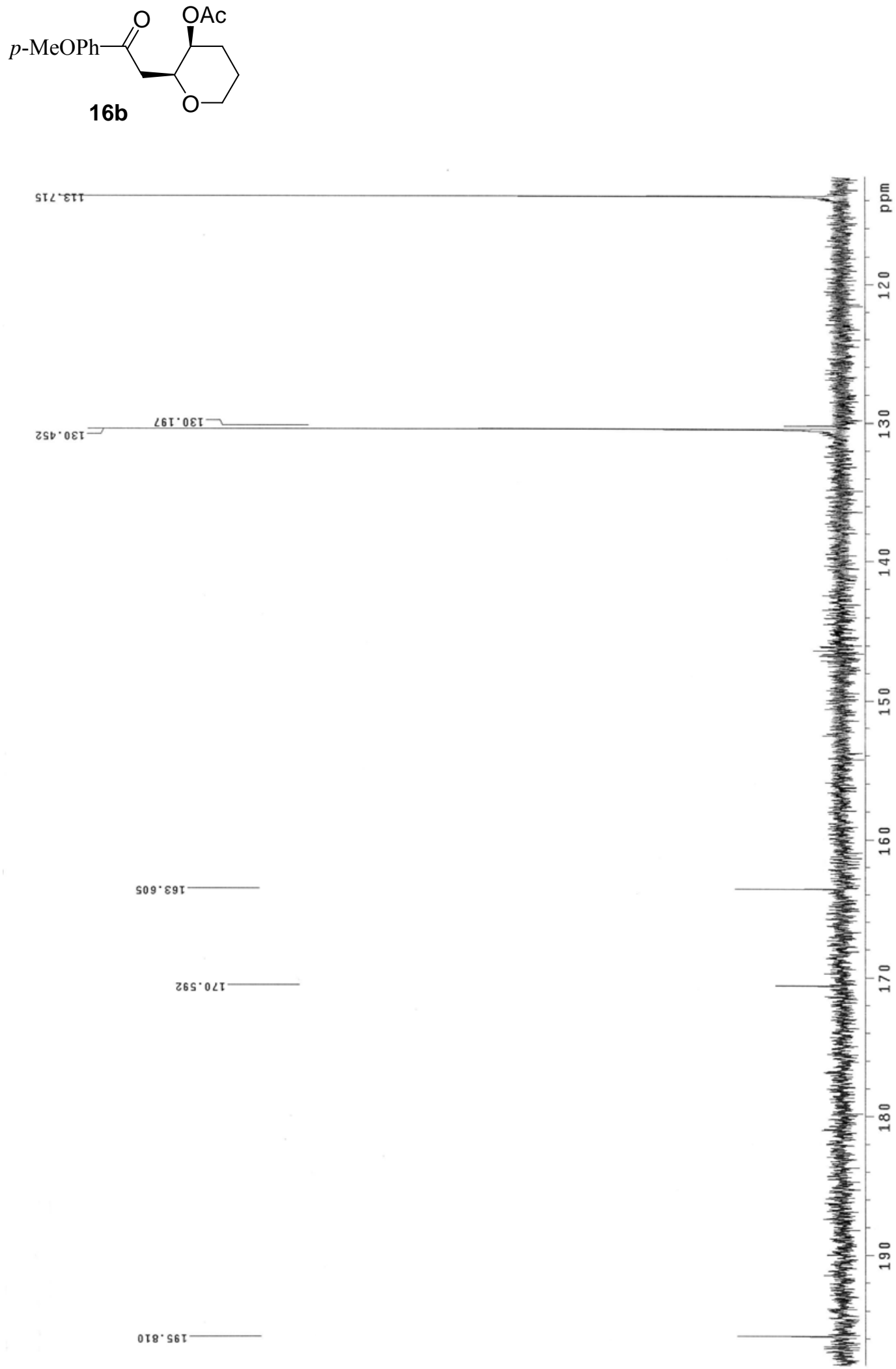

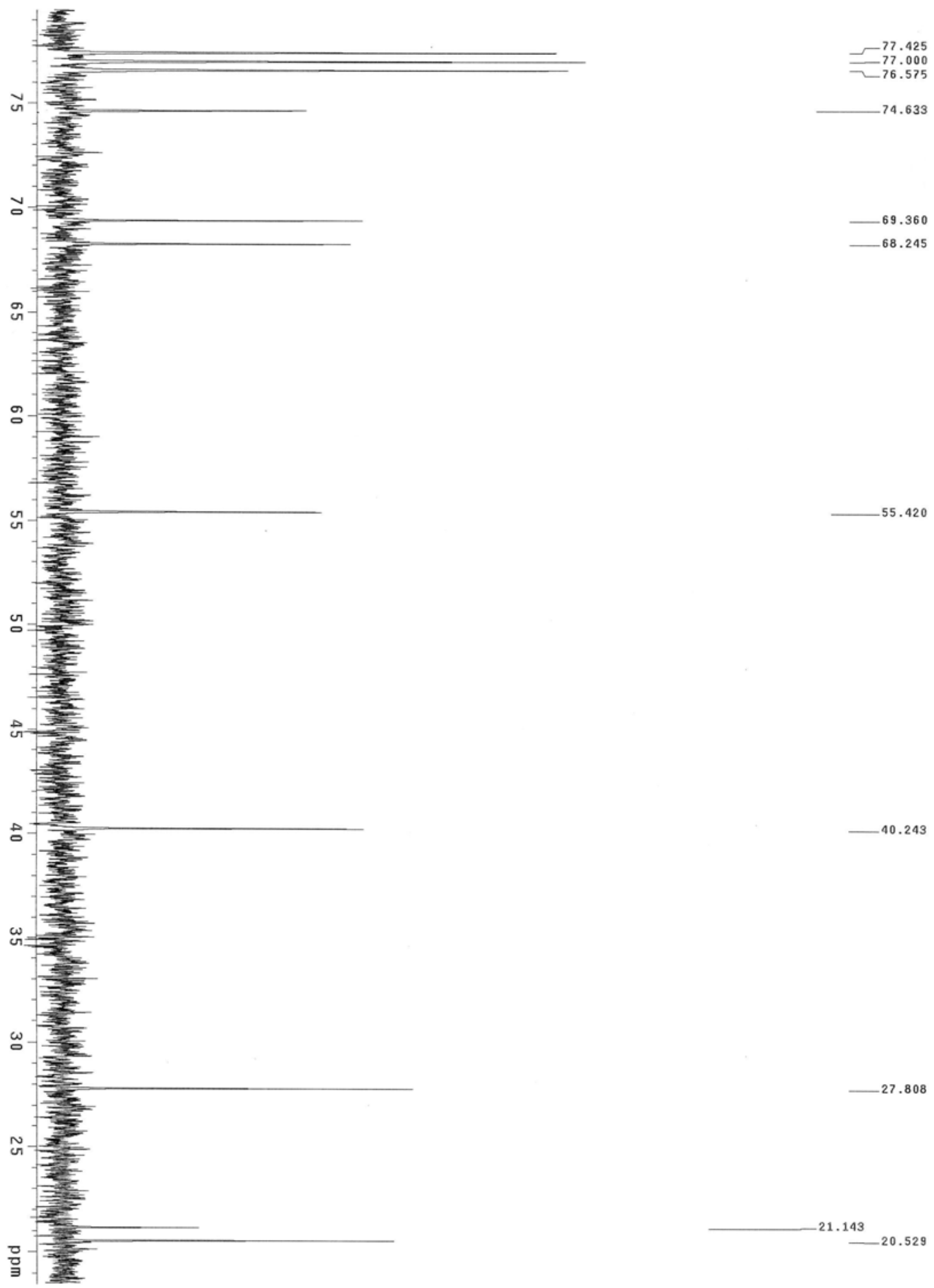

$-69.360$ 

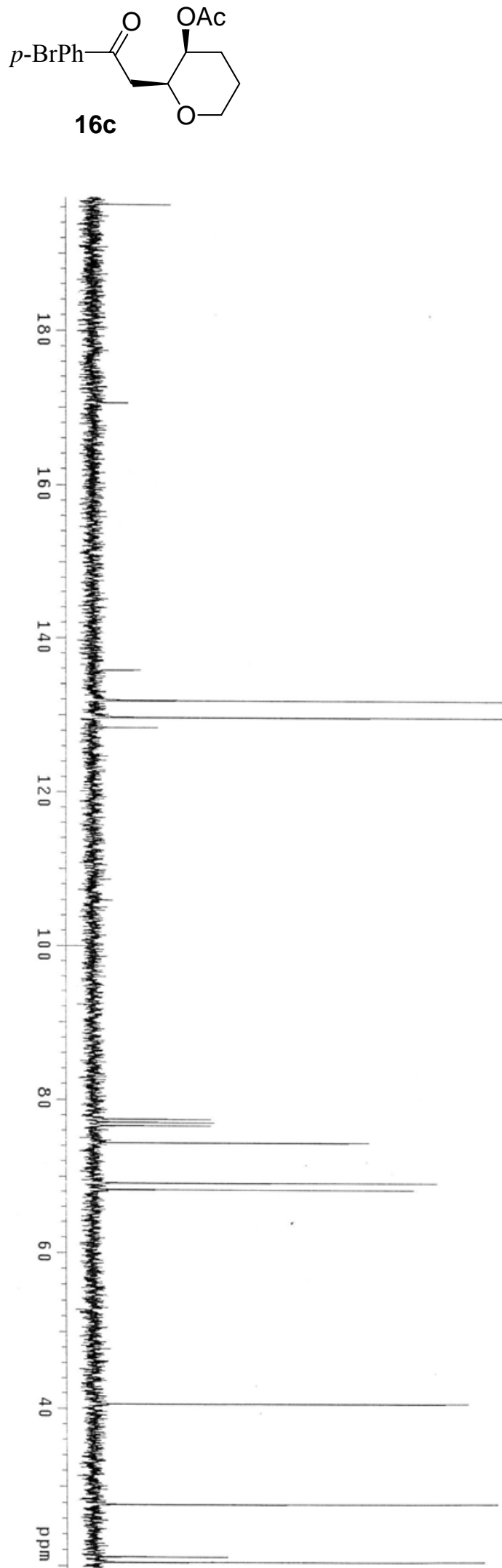

$-135.70$ 
naphthyl

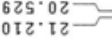

$828 \cdot 22$

$80 L^{\circ} 0 b$

$\angle 82 \cdot 89=$

$92 \varepsilon^{\circ} .69$

bT 9.62

$000 \cdot 24$

เ12. 24

$\angle 88^{\circ} \varepsilon z \mathrm{~T}$

$20 \angle \cdot \angle 2 \mathrm{~T}$

$\angle 80^{\circ} \cdot 82 \mathrm{I}$

$260 \cdot 821$

(665.

$\angle \varepsilon^{\circ}, \Delta \varepsilon \mathrm{I}$

ธรด. $ง 8 \mathrm{~T}$

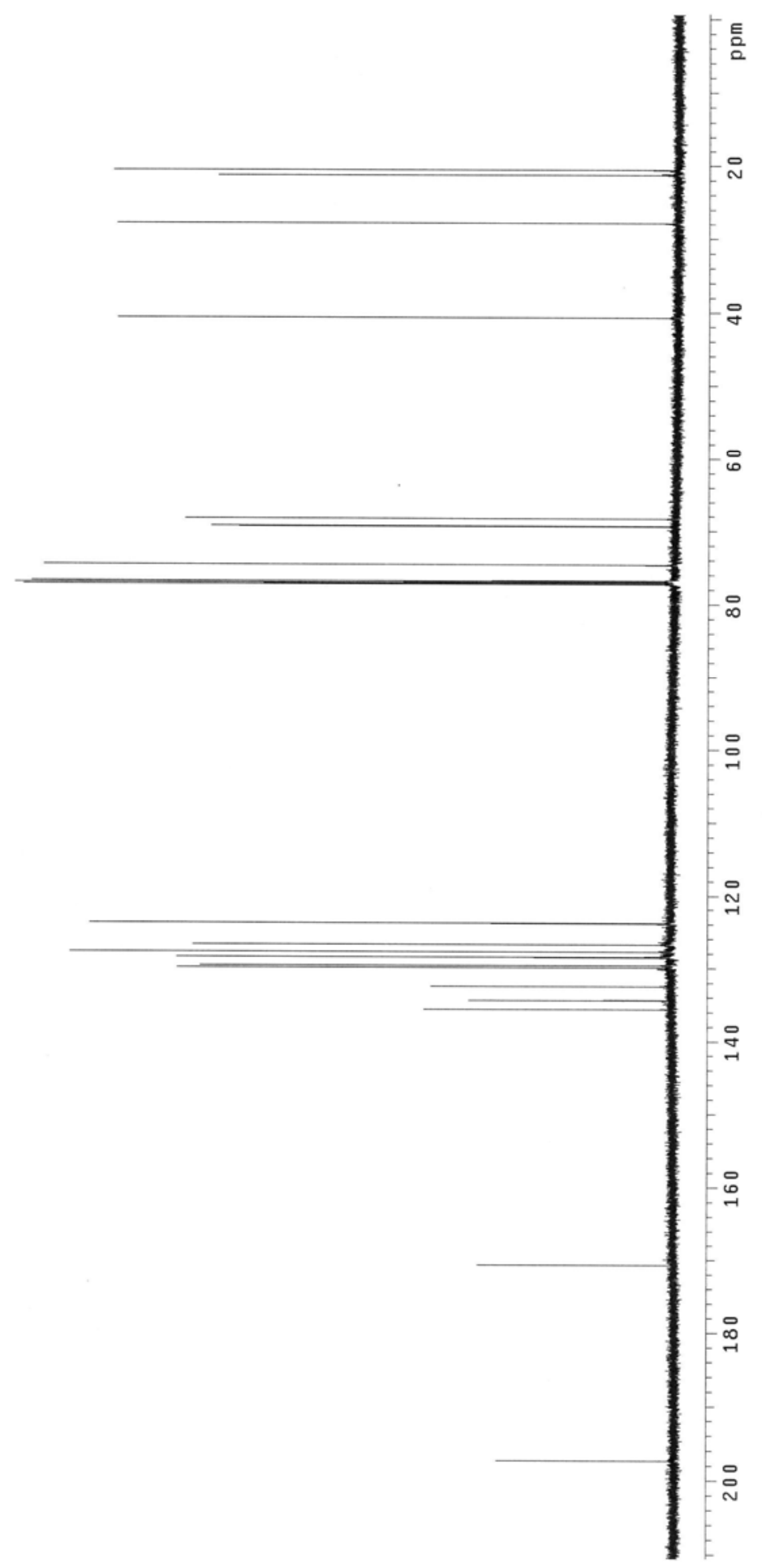




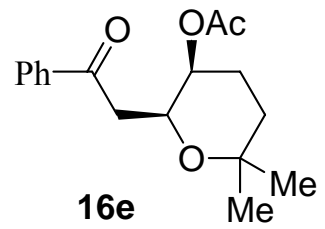

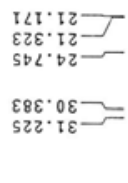

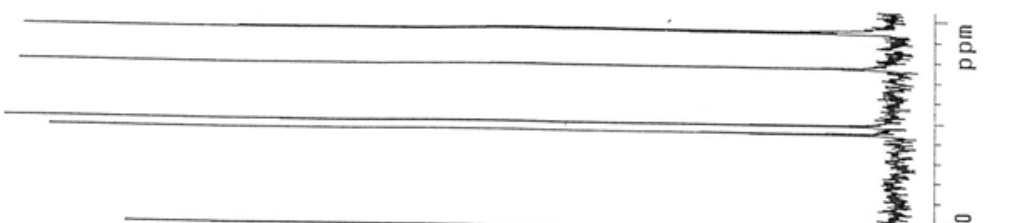

$906.00-$

$962^{\circ} \angle 9==$
$525^{\circ} 89=$

$2<\varepsilon \cdot 2<$

$0<5^{\circ} 927$

$\angle 89 \cdot \angle L$

$289^{\circ} 0<\tau$ 
S46

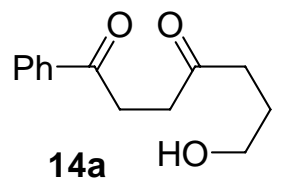

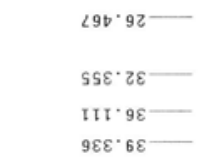
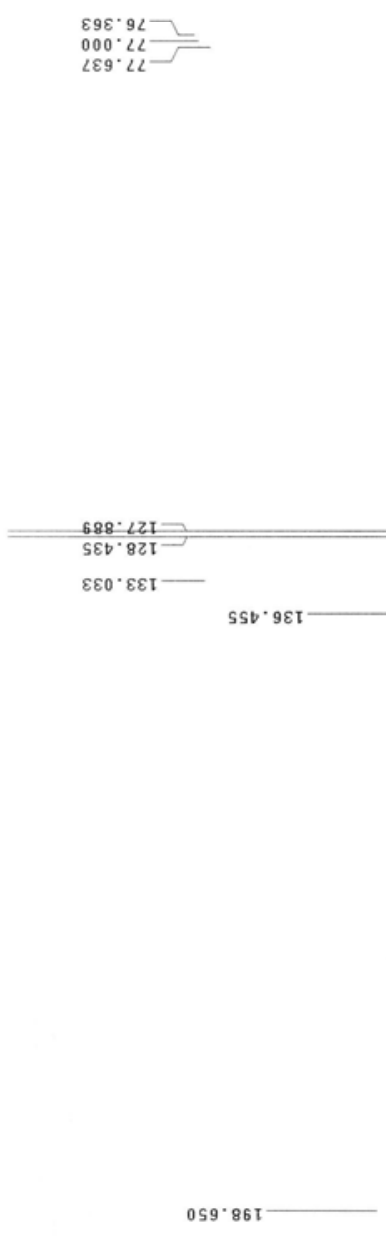

$D 68^{\circ} 602$

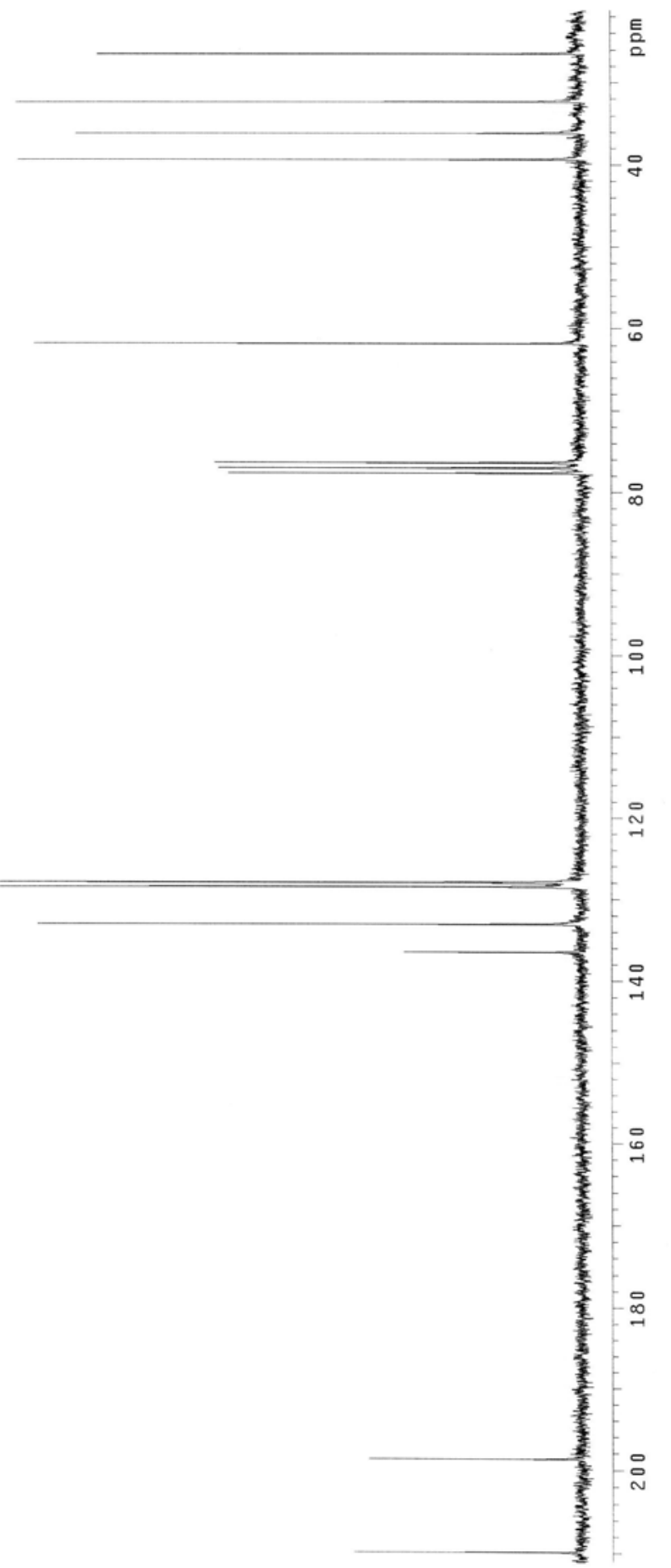



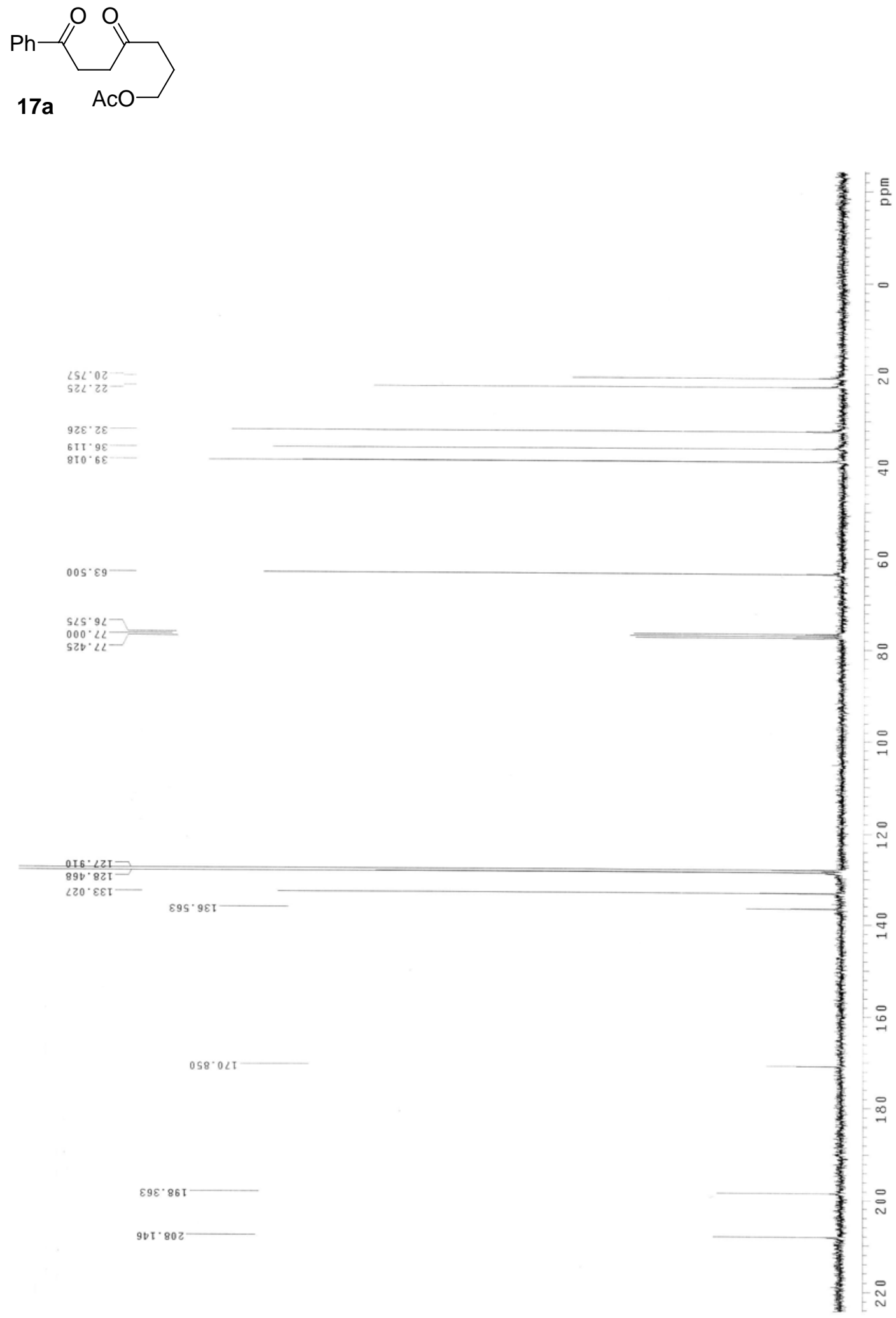
S48
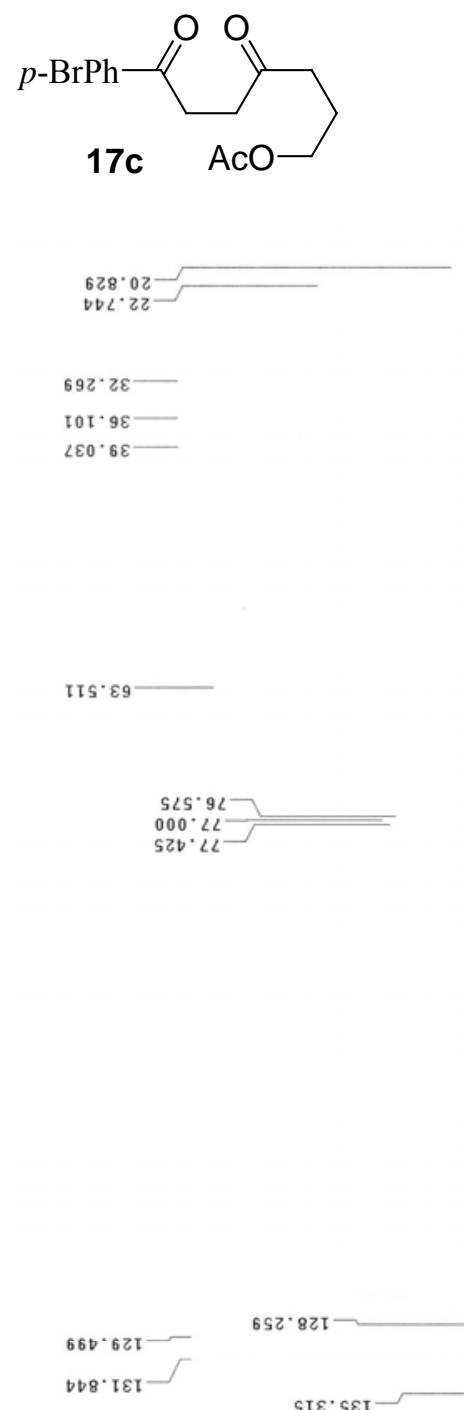

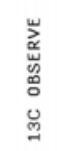

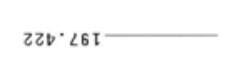

660.802

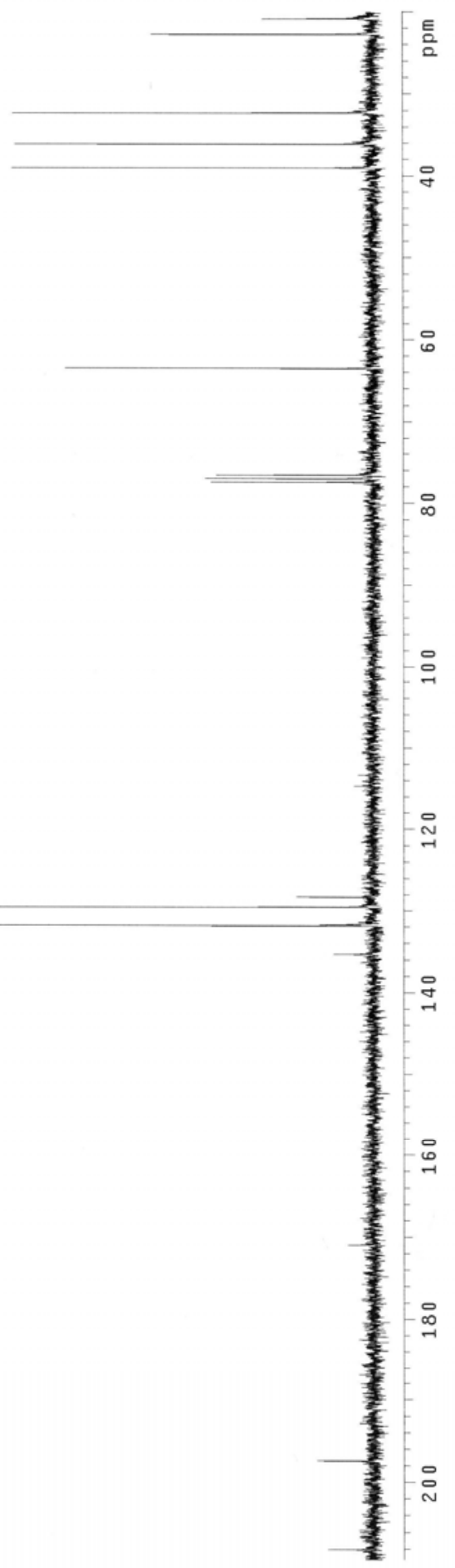


naphthyl $\left\langle\left.\right|_{\text {17d }} ^{O}\right\rangle$

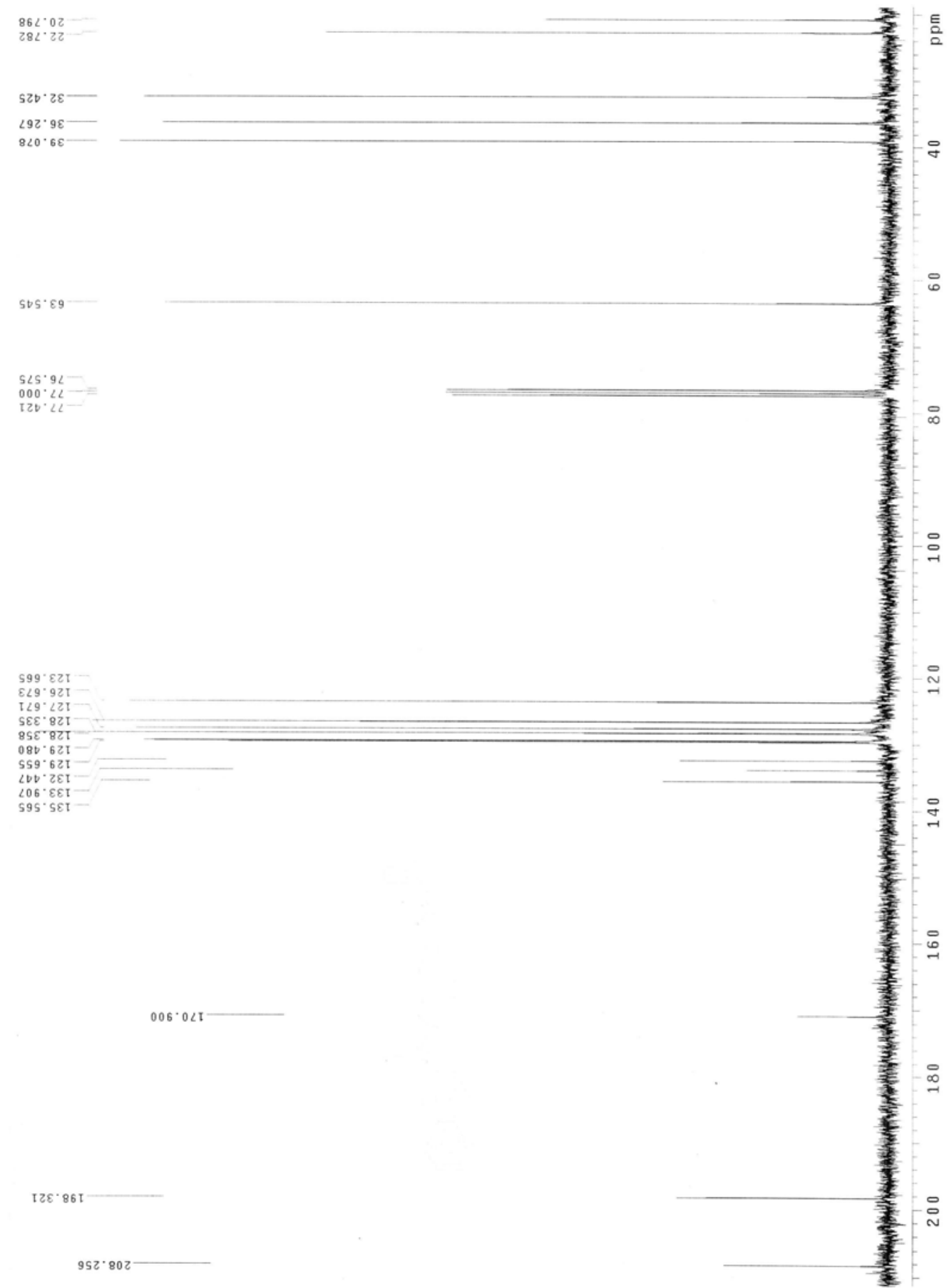




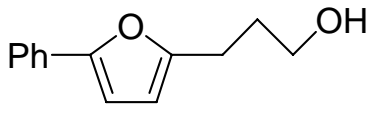

$18 \mathrm{a}$

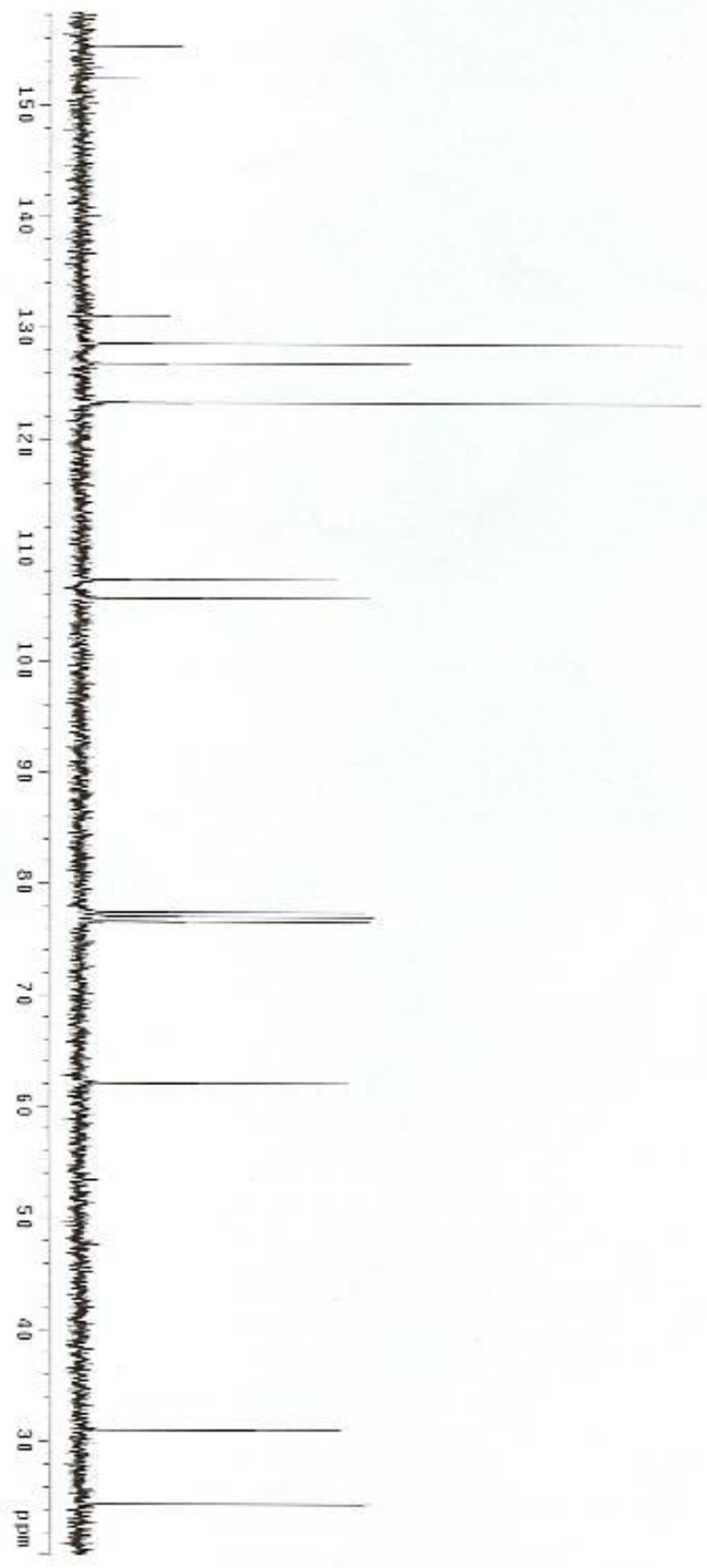

${ }^{-152.981}$

191.047

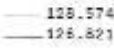

123.305

107.286
-105.636

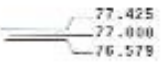

$-52.054$

31.006 

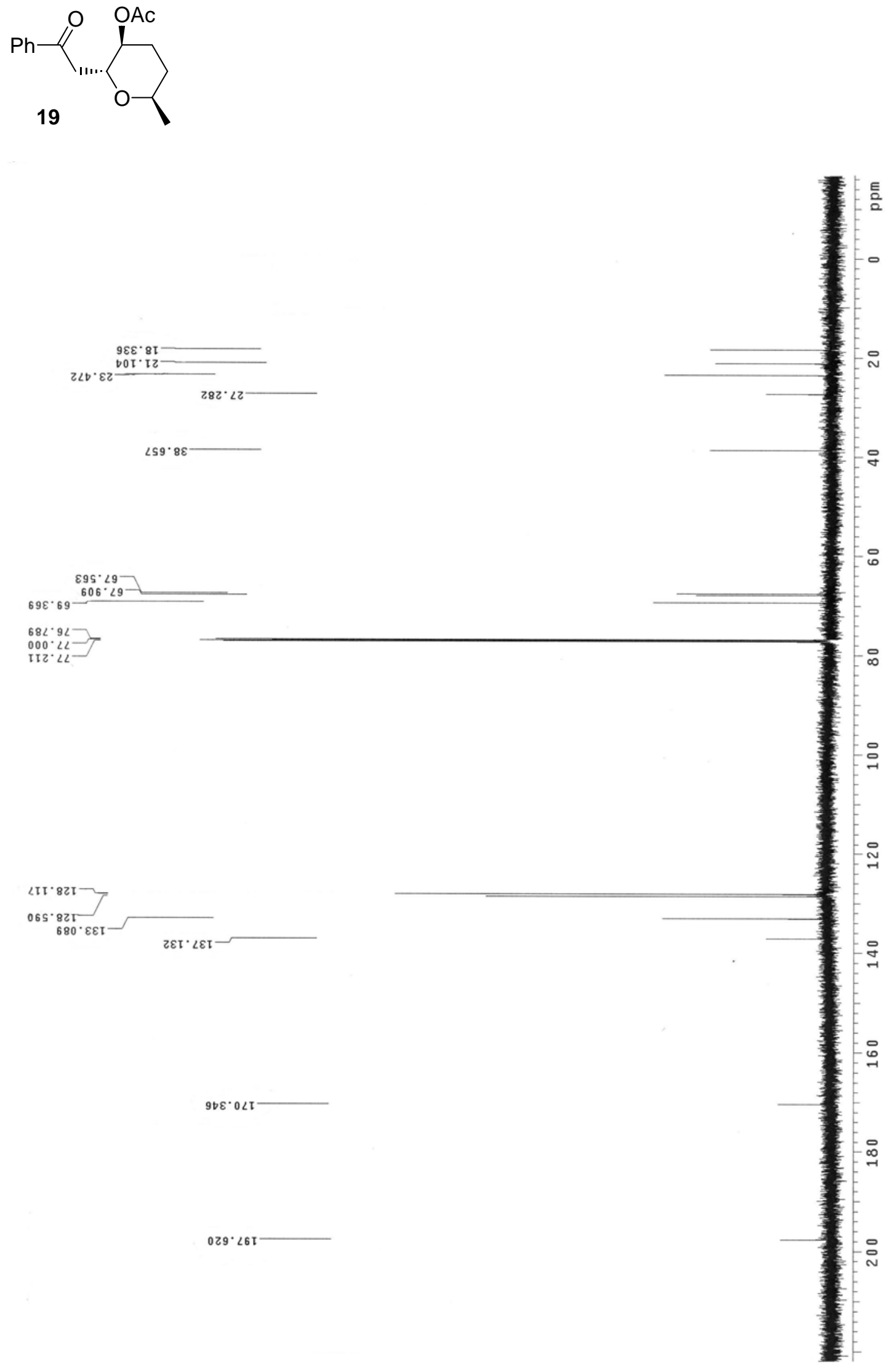

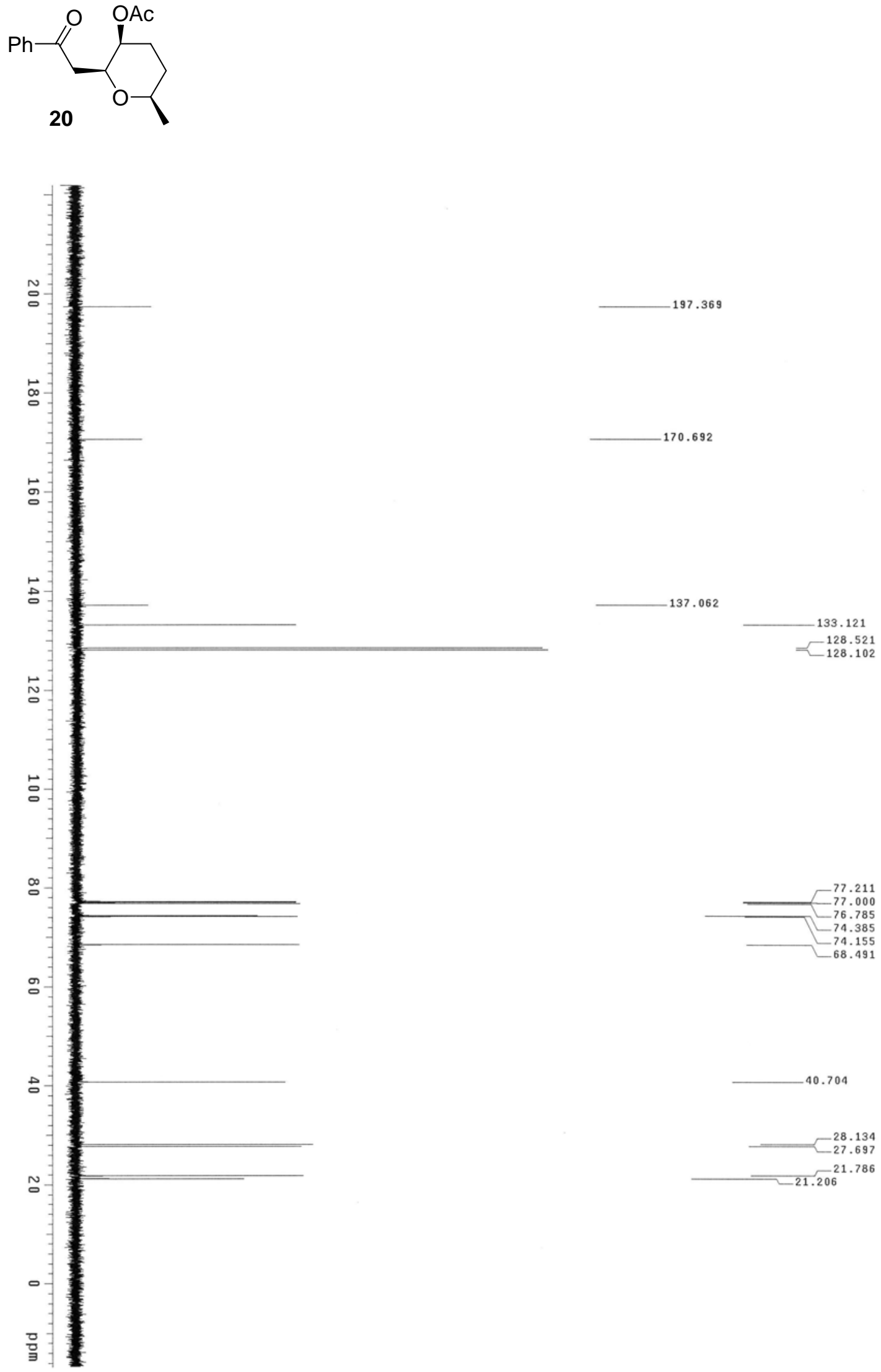
<smiles>CCCC1O[C@H](C)CC[C@H]1OC(C)=O</smiles>

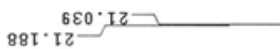

$6 \varepsilon v \cdot z \varepsilon \frac{090 \cdot 6 z-}{\square}$ $S \angle L \cdot I b-$

$88 I^{\circ} Z L-$
$\mathrm{SSL} \cdot \varepsilon L-$

$588^{\circ} \cdot 52-$

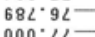

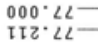

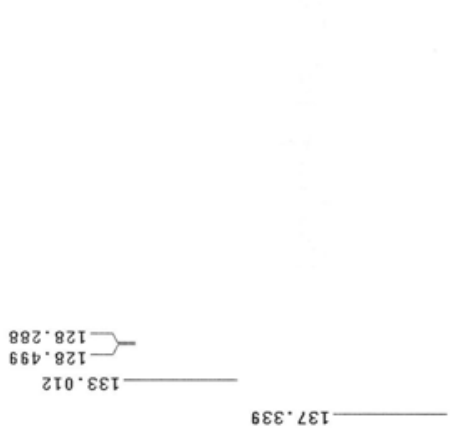

$\angle S E^{\circ} 0 \angle \mathrm{T}$

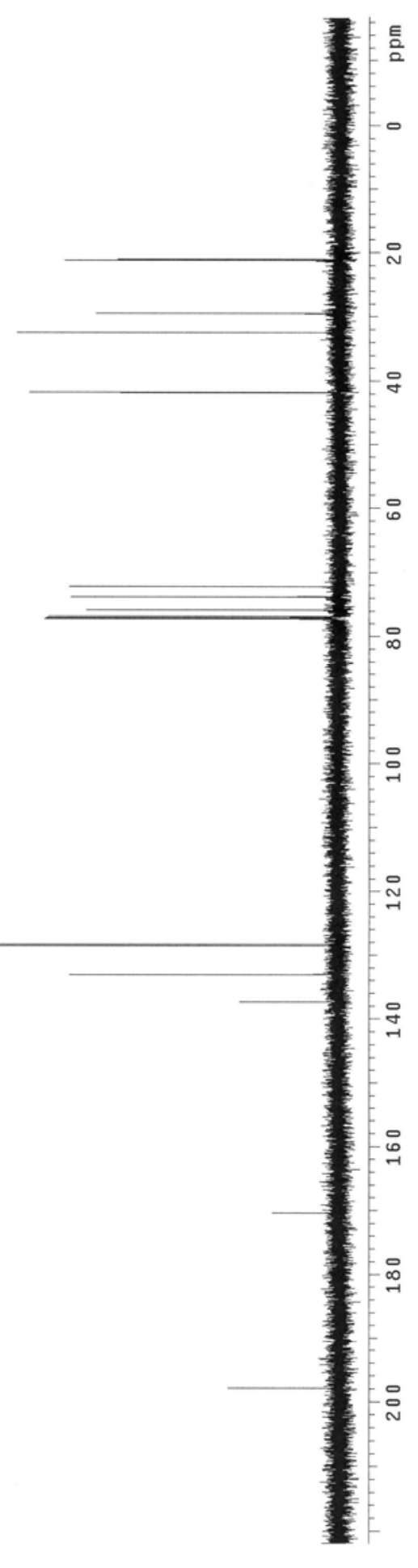

$6 \angle 8^{\circ} \angle 6 I$ 


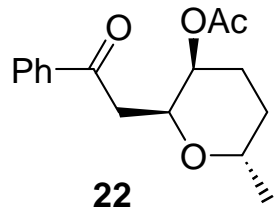

$2 \mathrm{I} 6.8 \mathrm{~T}$
$\mathrm{D} 9 \mathrm{~T} \cdot \mathrm{IZ}-$

$296.82=$

$950.82-$

$220 \cdot 00-$

$862 \cdot 09$
$\angle 60 \cdot 0<$
$1 \angle 2 \cdot 0<$

$682 \cdot 92$ 二

II 202

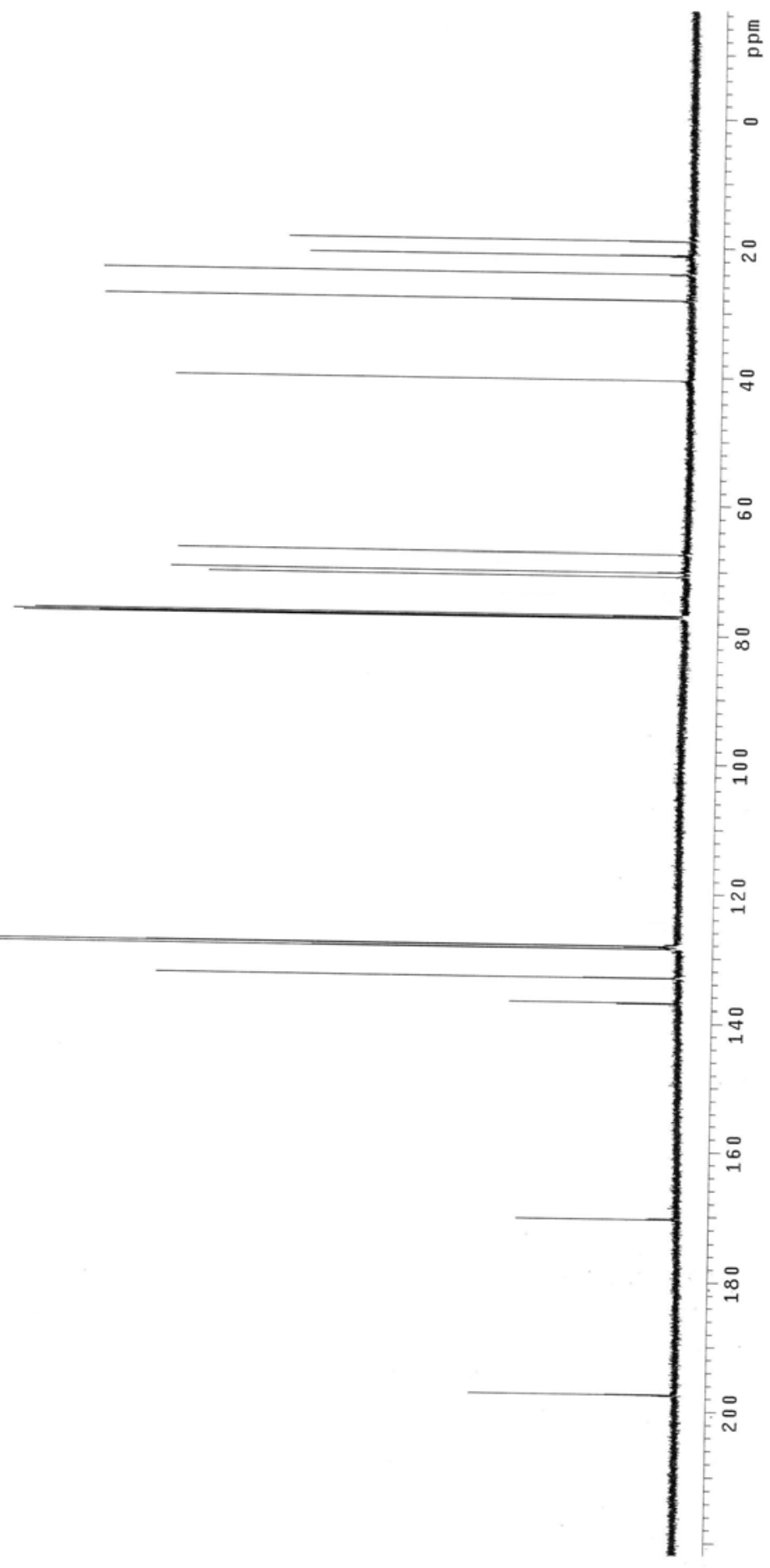




$$
1
$$


S56

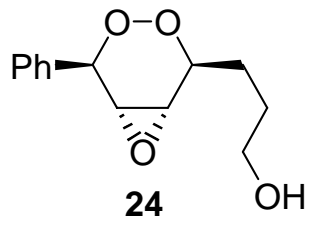

$586 \cdot 92-\square$
$\tau 8 \varepsilon \cdot 82-$

$820^{\circ} 25=$
$\angle S 2 \cdot 25=$

$500.29-$

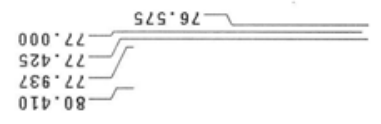

$S 8 L \cdot S \& \mathrm{~L}$

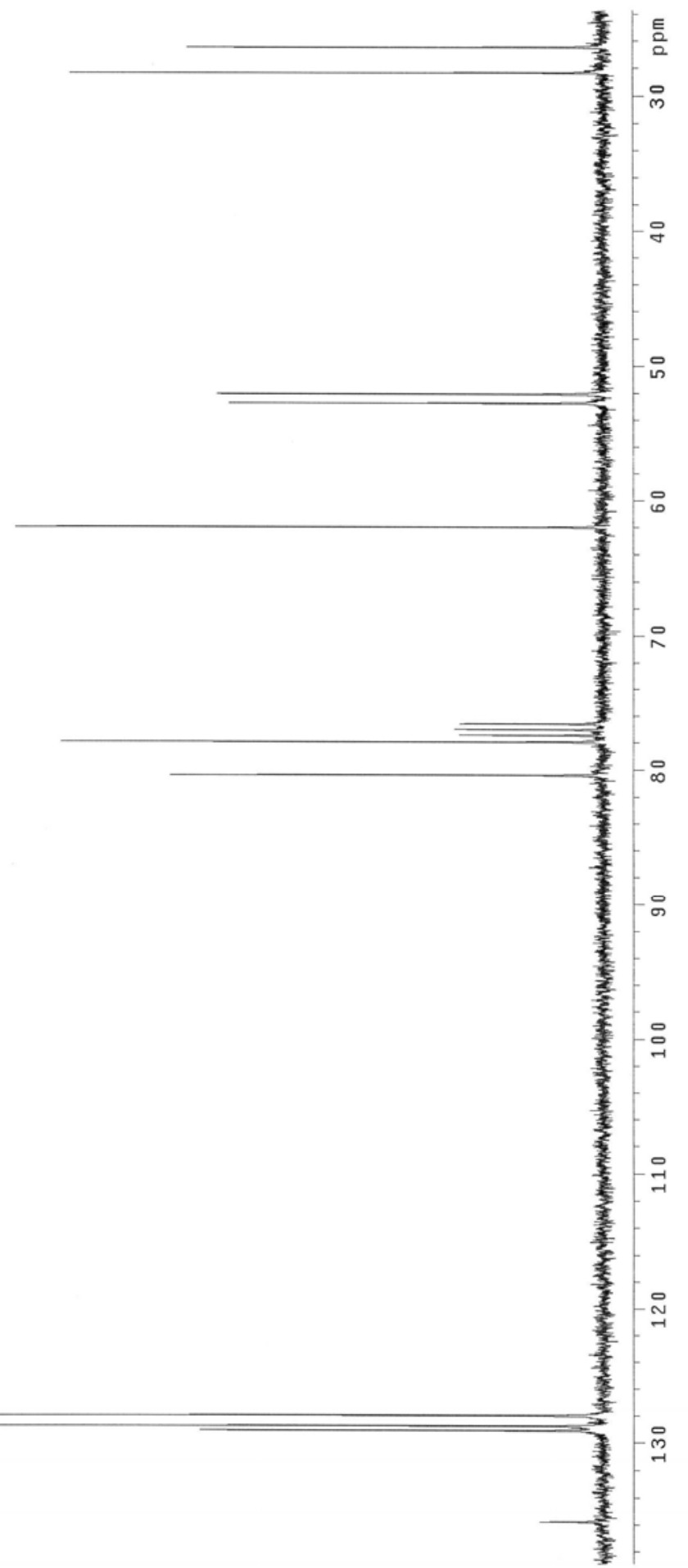



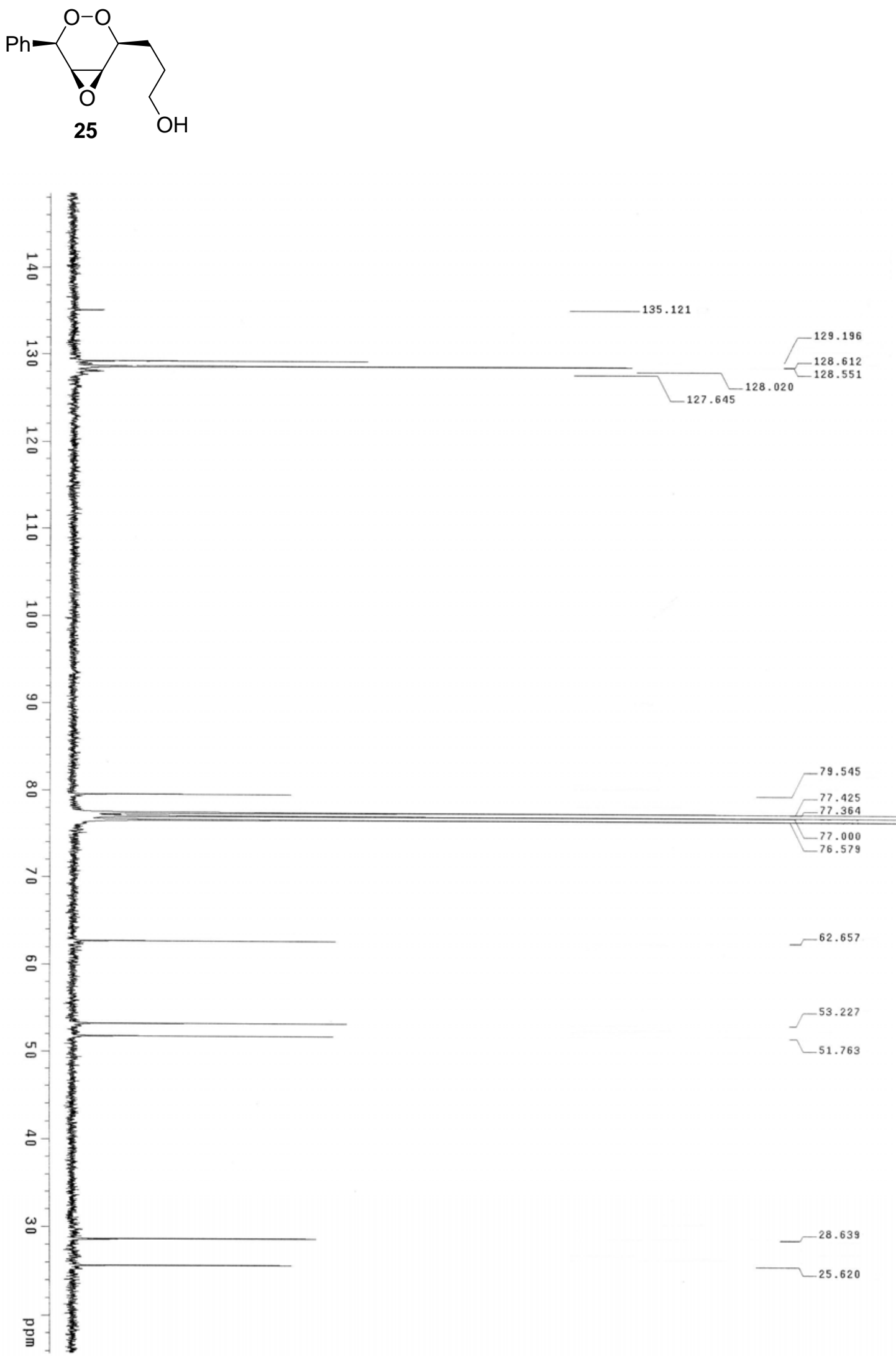

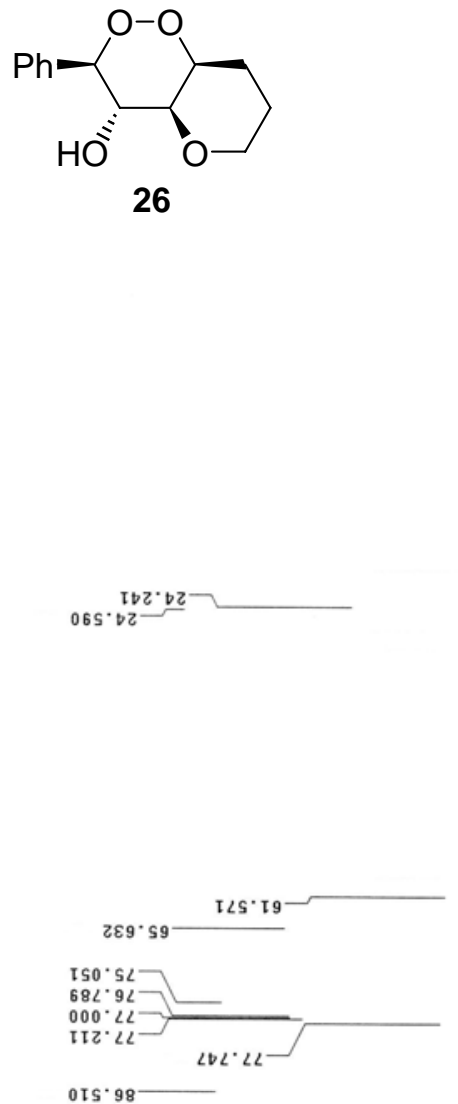

629.2217 985.821
098.821 B. . 581

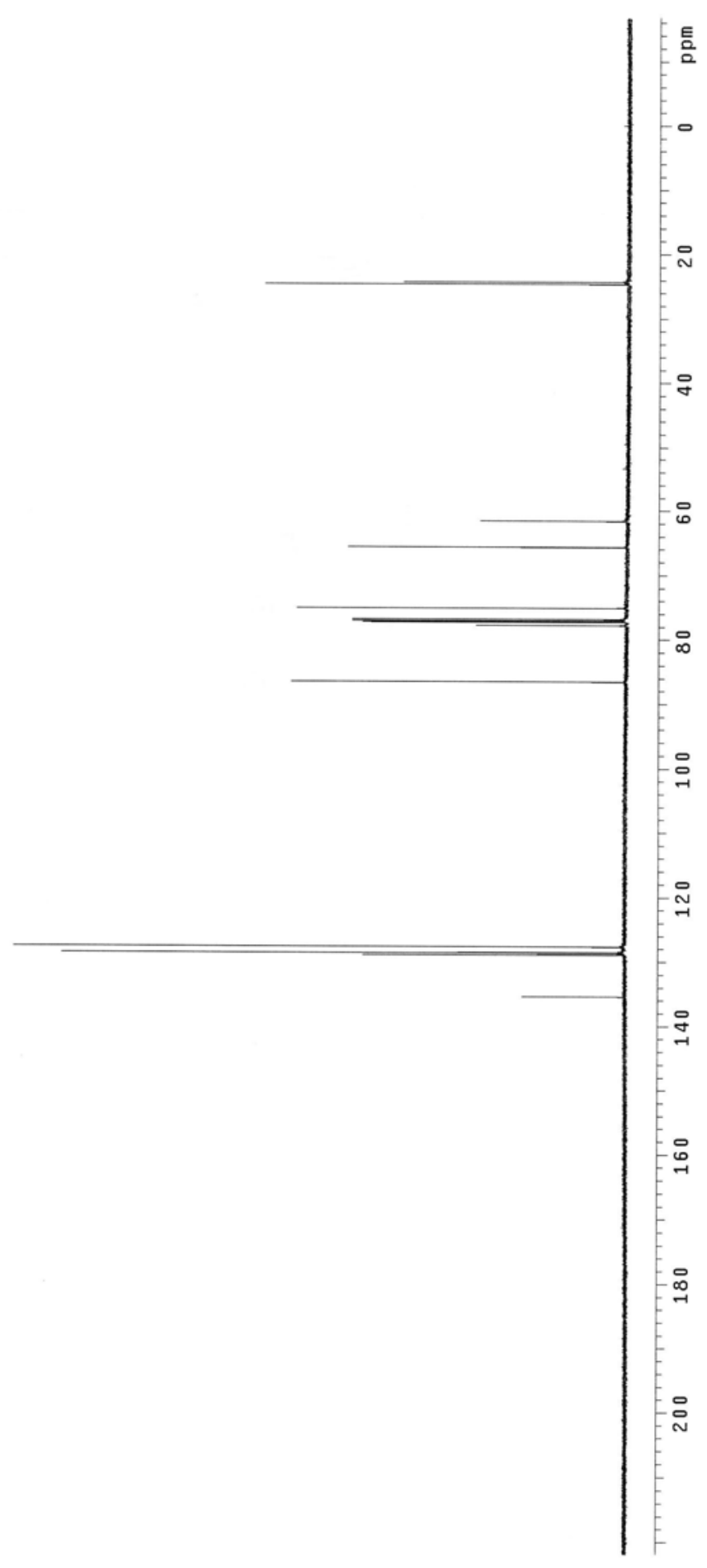



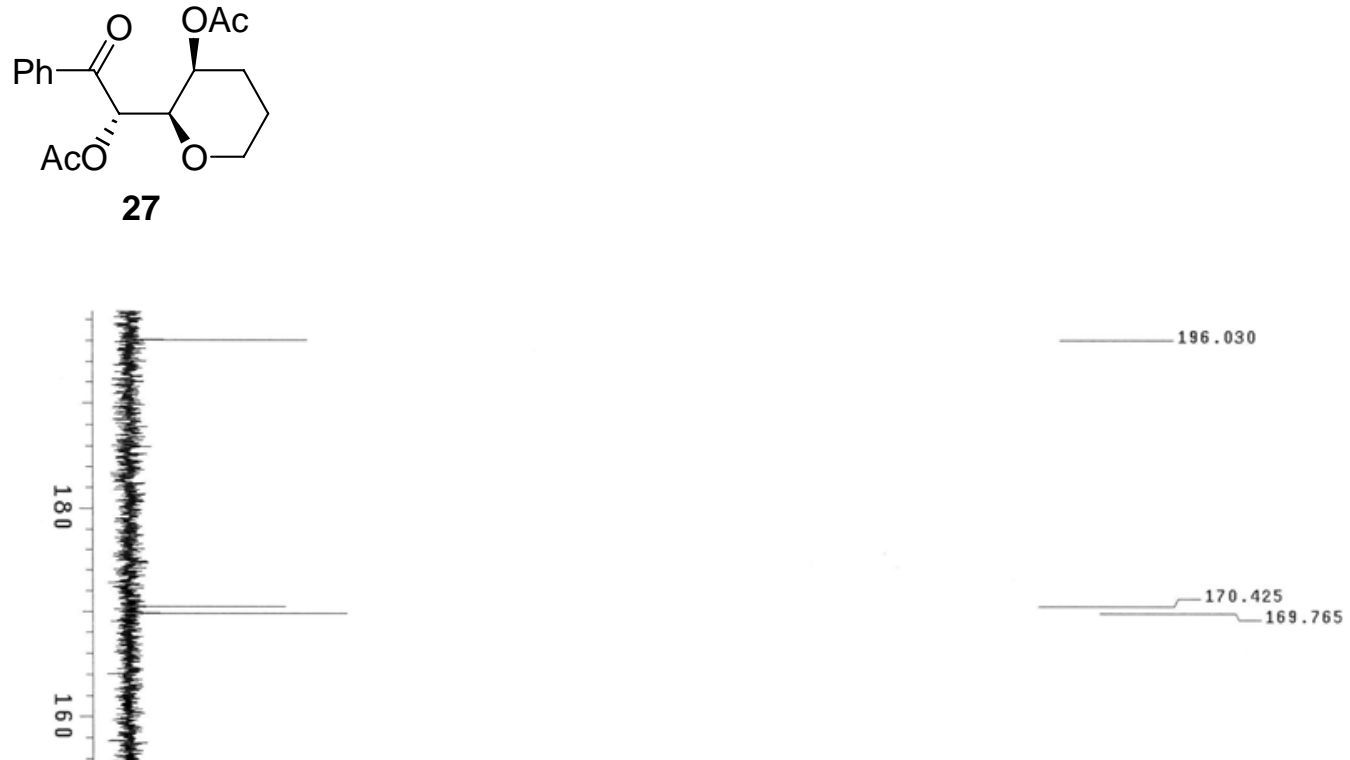

$\stackrel{\bullet}{\circ}$

$\stackrel{\leftarrow}{n}-$

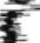
-133.748
$\sim-128.919$
-128.661

$\stackrel{\infty}{\circ}$

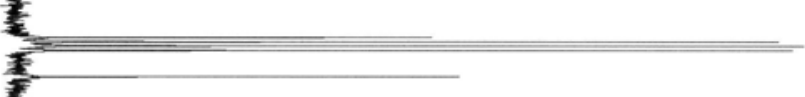

$\begin{array}{r}77.831 \\ -77.425 \\ \hline\end{array}$ -77.425
-77.000 77.575
-74.056

$\begin{array}{r}68.533 \\ -\quad 66.853 \\ \hline\end{array}$

욤

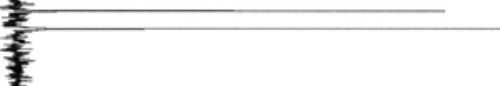

ㅁ.

뮼

27.285
-20.757 20.757
-20.597 $-20.597$ 当 

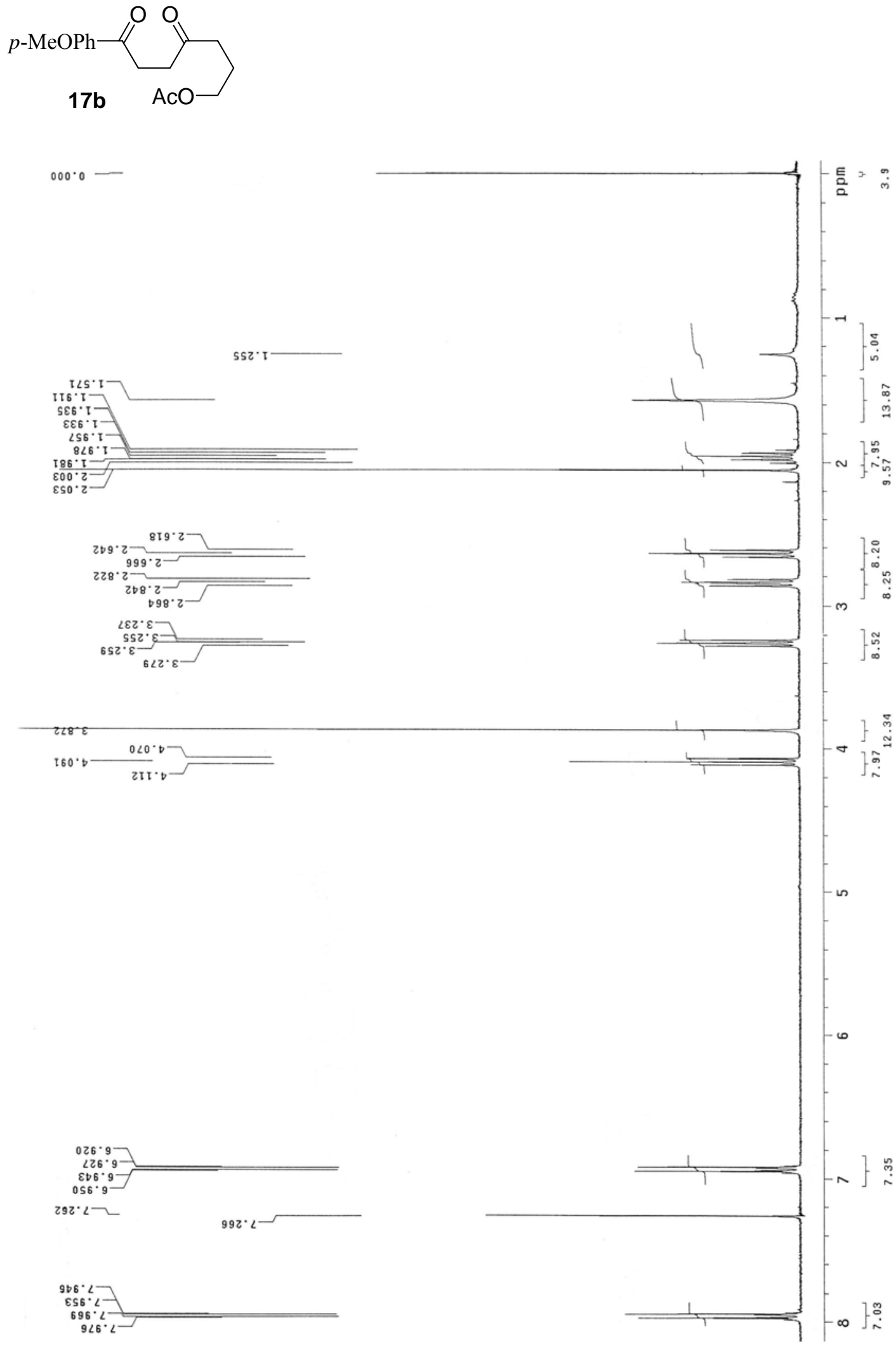